\title{
STUDY OF THE SEISMIC ATTENUATION GENERATED BY THE MUD LAYER IN LAKE MARACAIBO, VENEZUELA
}

\author{
A Thesis \\ by \\ JAVIER ANTONIO PEREZ ARREDONDO
}

\begin{abstract}
Submitted to the Office of Graduate Studies of Texas A\&M University in partial fulfillment of the requirements for the degree of

MASTER OF SCIENCE
\end{abstract}

May 2003

Major Subject: Geophysics 


\title{
STUDY OF THE SEISMIC ATTENUATION GENERATED BY THE MUD LAYER IN LAKE MARACAIBO, VENEZUELA
}

\author{
A Thesis \\ by \\ JAVIER ANTONIO PEREZ ARREDONDO \\ Submitted to Texas A\&M University \\ in partial fulfillment of the requirements \\ for the degree of \\ MASTER OF SCIENCE
}

Approved as to style and content by:

Richard L. Gibson

(Chair of Committee)

Joel S. Watkins

(Member)

Thomas A. Blasingame

(Member)

\begin{tabular}{c}
\hline $\begin{array}{c}\text { Joel S. Watkins } \\
\text { (Member) }\end{array}$ \\
\hline Andrew Hajash Jr. \\
(Head of Department)
\end{tabular}

May 2003

Major Subject: Geophysics 


\author{
ABSTRACT \\ Study of the Seismic Attenuation Generated by the Mud Layer in \\ Lake Maracaibo, Venezuela. (May 2003) \\ Javier Antonio Pérez Arredondo, B.S., Universidad del Zulia \\ Chair of Advisory Committee: Dr. Richard L. Gibson Jr.
}

Several seismic properties of Lake Maracaibo are unique and difficult to understand. However, studies show that the two principal factors that affect the seismic data are likely to be the low compressional and shear wave velocities generated by the gassy sediment in the mud layer, and the high attenuation of the compressional and shear waves. This mud layer sediment is heavy and is not suspended in the water. Furthermore, it is compacted enough to support shear stresses and, therefore, has a finite shear wave velocity.

In theory, the gas content of the sediment reduces the compressional wave velocity by an order of magnitude below the values for water saturated sediments, but in Lake Maracaibo several studies show that gassy sediment reduces the compressional wave velocity from $1500 \mathrm{~m} / \mathrm{s}$ to between $300 \mathrm{~m} / \mathrm{s}$ and $700 \mathrm{~m} / \mathrm{s}$. This situation produces high attenuation of the compressional waves that are traveling through the sediment in the first $100 \mathrm{~m}$. However, the results of seismic modeling show that this velocity has to be between $700 \mathrm{~m} / \mathrm{s}$ and $900 \mathrm{~m} / \mathrm{s}$ in order to get reasonable results, at least in the study area.

The results show that there are very important lithological differences between the zones with and without gassy sediments in the mud layer. The best match between the raw data and the synthetic seismogram was found when an embedded rigid shale layer was located within the mud layer, in the first $100 \mathrm{~m}$. Unrealistic results were produced when the rigid shale layer was removed in the modeling. This rigid layer produces a destructive interference in the Stoneley wave that could be observed in the seismic data and the synthetic seismogram.

In this research, the attenuation quality factor $Q$, an intrinsic property of rock, will be studied. Common attenuation mechanisms include grain sliding, viscous flow of pore fluid or gas, viscous relaxation and other features. Additionally, it will be shown that other factors can be proposed to account for the attenuation of compressional and shear waves in Lake Maracaibo sediments. They include: the viscous losses 
between the particles and the fluid immediately above the mud layer; and the solid friction losses between the particles, the mud layer and the gassy sediment. This research shows that the attenuation in the mud layer in the zone with gassy sediment is very different from the attenuation in the zone without gassy sediment, and that the compressional wave attenuation is larger in the mud layer zone with gassy sediment than in the mud layer zone without gassy sediment. Finally, the research shows that the ringing is an important phenomenon associated with the low velocity in the mud layer and that this ringing has more frequency content in the zone without gassy sediment than in the zone with gassy sediment. 
To my wife Gloria Patricia 


\section{ACKNOWLEDGMENTS}

I would like to thank Dr. Richard Gibson for allowing me the oportunity to work with him. His guidance and advice were extremely helpful in completing my thesis project.

I would like to thank Dr. Joel S. Watkins and Dr. Thomas A. Blasingame for their help and support. Much appreciation is extended to Dr. Gibson's students: Constantinos Tzimeas, Sung Hwan Yuh and Jin Lee Kyoung for their help and patience.

I would also like to thank Petroleos de Venezuela S.A. (PDVSA) for its financial support and allowing me to complete this Master of Science degree at Texas A\&M University. 


\section{TABLE OF CONTENTS}

ABSTRACT $\ldots \ldots \ldots \ldots \ldots \ldots \ldots \ldots \ldots \ldots \ldots \ldots \ldots \ldots$ ii

DEDICATION ................... v

ACKNOWLEDGMENTS $\ldots \ldots \ldots \ldots \ldots \ldots \ldots \ldots$ vi

TABLE OF CONTENTS $\ldots \ldots \ldots \ldots \ldots$ vii

LIST OF TABLES $\ldots \ldots \ldots \ldots \ldots \ldots \ldots \ldots \ldots \ldots \ldots \ldots \ldots \ldots \ldots$

LIST OF FIGURES $\ldots \ldots \ldots \ldots \ldots \ldots \ldots \ldots \ldots \ldots \ldots \ldots \ldots$ CHAPTER

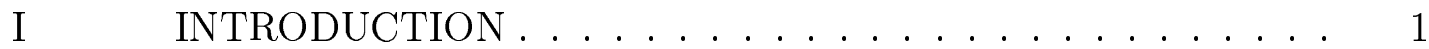

II $\quad$ BASIC THEORY $\ldots \ldots \ldots \ldots \ldots \ldots \ldots \ldots$

2.1 Compressional and Shear Waves' Speed . . . . . . . 5

2.1.1 Unconsolidated Sediments . . . . . . . . . . . . 6

2.1.2 Consolidated Sediments _. . . . . . . . . 12

2.1.3 Gassy Sediments . . . . . . . . . . . . 15

2.2 Attenuation of Compressional and Shear Waves . . . 18

2.2.1 Unconsolidated Sediments . . . . . . . . . . 25

2.2 .2 Consolidated Sediments _ . . . . . . . . . 28

2.2 .3 Gassy Sediments . . . . . . . . . . . 30

2.3 Attenuation Estimation . . . . . . . . . . . . . . 31

2.4 Gas Content Estimation . . . . . . . . . . . . 35

III LAKE MARACAIBO FEATURES . . . . . . . . . . . 39

3.1 Geographical Location and Geology . . . . . . . . . . 39

$3.2 \quad$ Seismic Features . . . . . . . . . . . . . . . 49

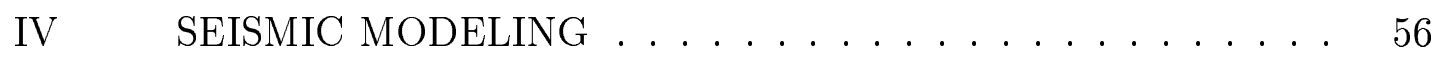

4.1 Modeling Without Gassy Sediment . . . . . . . . 57 
4.2 Modeling With Gassy Sediment . . . . . . . 73

$\mathrm{V} \quad$ ANALYSIS AND RESULTS . . . . . . . . . . . 91

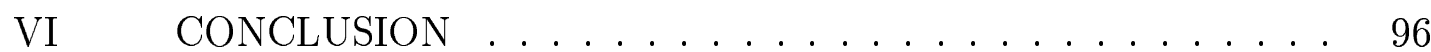

REFERENCES . . . . . . . . . . . . . . . . . 99

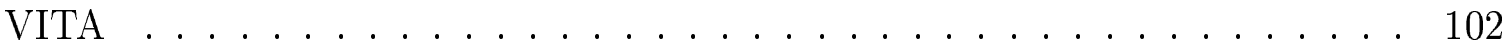




\section{LIST OF TABLES}

TABLE

2.1 Relationships between compressional wave speed and physical properties of unconsolidated sediments, without gas bubbles (Shumway,

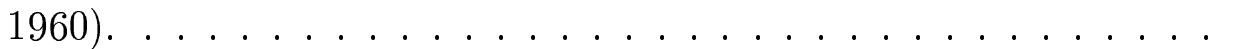

2.2 Principal attenuation factors in unconsolidated sediments, consolidated sediments and gassy sediments observed in Lake Maracaibo due to the mud layer zone. . . . . . . . . . . . . . . .

2.3 Typical values for the bulk modulus of the fluid, hydrostatic pressure, fluid velocity and gassy sediment velocity. The effective gas content, when the gassy sediment velocities are $300 \mathrm{~m} / \mathrm{s}$ and 700 $\mathrm{m} / \mathrm{s}$, are $1.89 \%$ and $0.18 \%$ respectively (Levin, 1962). . . . . . 38

3.1 Gas content in the recent mud sediments. The samples were taken in the northeast portion of the lake [Core No. 1] and in the center of the lake [Core No. 2] (Sarmiento and Kirby, 1962). . . . . . . . .

4.1 Relationships between compressional wave quality factor $Q_{\alpha}$ and shear wave quality factor $Q_{\beta}$ versus sediment types. This relationship was used to control the quality factor $Q$ used in the different synthetic seismograms (Lay and Wallace, 1995; Hamilton, 1972; Bowles, 1997). . . . . . . . . . . . . . .

4.2 The different parameters used in the building of the synthetic seismograms in the mud layer zone without gassy sediments in Lake Maracaibo. ...................

4.3 The different parameters used in building of the synthetic seismograms in the mud layer zone with gassy sediments in Lake Maracaibo.

5.1 Final results found in the modeling of the mud layer without gassy sediment in Lake Maracaibo. The results show several differences from the zone with gassy sediment. 
TABLE

5.2 Final results found in the modeling of the mud layer with gassy sediment in Lake Maracaibo. The results show that there are several differences compared to the zone without gassy sediment. . . 


\section{LIST OF FIGURES}

FIGURE

Page

2.1 Compressional wave speed versus porosity for unconsolidated marine sediments. Data for 110 samples were used to show the relationship of porosity to compressional wave speed (Shumway, 1960). .

2.2 Compressional wave speed versus suspended particle volume concentration. In this case the estimation was made using a suspension of kaolinite clay water (Anderson and Hampton, 1980a). . . . .

2.3 Relationship between mean particle diameter and porosity for unconsolidated marine sediments without gas bubbles. The correlation coefficient is 0.95 (Shumway, 1960). . . . . . . . . .

2.4 Relationship between sediment particle diameter and compressional wave speed for unconsolidated marine sediments without gas bubbles (Shumway, 1960). . . . . . . . . . . .

2.5 Compressional wave speed versus gas content, in percentage, using one atmosphere pressure. The porosity was between 0.35 to 0.85. The frame elastic properties were included (Anderson and Hampton, 1980b). . . . . . . . . . . . . .

2.6 Compressional wave speed ratio in gassy sediment versus frequency ratio. Ocean bottom silt sediment and harbor mud sediment were used to calculate compressional wave velocity (Anderson and Hampton, 1980b). . . . . . . . . . . . .

2.7 Attenuation of compressional waves versus frequency in natural, saturated sediments and sedimentary strata. The line labeled $f^{1}$ indicates the slope of any line representing a first power dependence of attenuation on frequency (Hamilton, 1980). . . . . . . . .

2.8 Attenuation in gassy sediments versus frequency ratio. This frequency ratio is the frequency over the bubble resonance frequency. In this case two different sediments were used (Anderson and

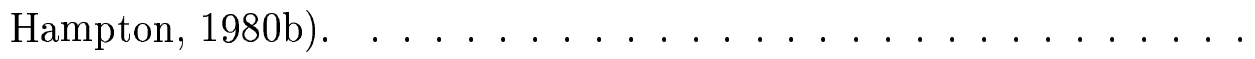


FIGURE

2.9 Three different shot gathers from Lake Maracaibo. These seismic sections are from different seismic lines located in three different areas. The first location is an area without mud layer problems and the other two are in a zone with mud layer problems. Amplitude gain control was applied. . . . . . . . . . .

3.1 Geological map from Venezuela. Lake Maracaibo is in the northwestern corner of Venezuela. . . . . . . . . . . . . . .

3.2 Lake Maracaibo map. It is possible to observe the location of the three seismic lines used in mud layer study. The relative location of the mud layer zones are shown. . . . . . . . . . . . . .

3.3 Near surface cross section of Lake Maracaibo. This is a simplification of the different strata observed in the area. The gassy sediments are principally in the mud layer (Michelena et al., 1998). .

3.4 Estimation of the shear velocity in depth from the pile records. These velocities are averages for three different areas in Lake Maracaibo, where the mud layer is present. . . . . . . . .

3.5 Generalized stratigraphic column of recent sediments of Lake Maracaibo (Sarmiento and Kirby, 1962). The sediment studied included a clay substratum [Unit A], probably Pleistocene, and the recent sediments divided into four stratigraphic units. . . . . . .

3.6 Velocity profile used to build the synthetic seismogram in Lake Maracaibo for the mud layer zone. Three different kinds of attenuation mechanisms are considered: viscous losses, solid friction losses and gassy sediment effect. . . . . . . . . . . .

3.7 Shot gather from Lake Maracaibo. This seismic section is from the area without gassy sediment in the mud layer. Multiple reflections can be observed between $3 s$ and $4 s$, due to the reverberation produced by the bottom and the surface of the lake. . . . . . . .

3.8 Different types of ray paths from the source $\left(o, d_{1}\right)$ to $(x, z)$. These waves are bouncing back and forth within the layer because of nearly perfect reflectivity of the boundaries. . . . . . . . . . . 52 
4.1 Shot gather from Lake Maracaibo. This seismic section is from the area without gassy sediment in the mud layer. A basic processing was applied to the seismic data section that involved bandpass filter.

4.2 Frequency spectrum for the raw data, Line No. 1. The results show that the central frequency is close to $30 \mathrm{~Hz}$. However, in the synthetic seismogram $25 \mathrm{~Hz}$ was used as the central frequency. . . . .

4.3 CDP gather and velocity analysis made over the seismic section, from Lake Maracaibo, without gassy sediment in the mud layer. In this analysis the SU library was used. Only velocity information between $2 \mathrm{~s}$ and $4 \mathrm{~s}$ was available . . . . . . . . . . . . .

4.4 Velocity profile used to build the synthetic seismogram in Lake Maracaibo for the mud layer zone. Three different kinds of attenuation mechanisms are considered: viscous losses, solid friction losses and mud layer effect, without gassy sediment. This measures only the first $100 \mathrm{~m} \ldots \ldots \ldots \ldots \ldots$

4.5 Velocity profile used to build the synthetic seismogram in Lake Maracaibo for the mud layer zone. This configuration is for the deeper reflector. This configuration was used for both situations, the mud layer without gassy sediment and with gassy sediment, respectively. . . . . . . . . . . . . . .

4.6 Seismic acquisition configuration used to build the synthetic seismogram in Lake Maracaibo. This configuration was used in both cases; mud layer with the gassy sediment problem and without the gassy sediment problem, respectively. In this case, 150 receivers were used. An exaggerated vertical scale was used. . . . . . . . . .

4.7 Synthetic seismogram for Lake Maracaibo obtained using a layer configuration without gassy sediment and the seismic geometry acquisition split spread. The model configuration was composed with 13 layers. . . . . . . . . . . . . . . . . . . .

4.8 Seismic data set from Lake Maracaibo in the mud layer area without gassy sediment and the synthetic seismogram built for this zone. There are at least six similarities. . . . . . . . . . . 
4.9 Synthetic seismogram with the same layer configuration, velocities, thicknesses, densities, etc., but with different $n$ value, in the zone without gassy sediments. In the figure on the right, the value for $n$ was 1 ; on the left, the value for $n$ was $0 \ldots \ldots \ldots \ldots$

4.10 Synthetic seismogram, in the zone without gassy sediments, with the same layer configuration, velocities, thicknesses, densities, etc., but with the attenuation quality factor $Q_{\beta}$ equal to 100 in the vis-

4.11 Shot gather from Lake Maracaibo. This seismic section is from the area with gassy sediment in the mud layer, Line No. 2. A basic processing was applied to the seismic data section that involved bandpass filter. . . . . . . . . . . . . .

4.12 Frequency spectrum for the raw data, Line No. 2. The results show that the central frequency is close to $25 \mathrm{~Hz}$. It is possible to observe the different frequency content between this line and Line No. 3. . . . . . . . . . . . . . . . . . . . .

4.13 Shot gather from Lake Maracaibo. This seismic section is from the area with gassy sediment in the mud layer, Line No. 3. A basic processing was applied to the seismic data section that involved bandpass filter. . . . . . . . . . . . . . . .

4.14 Frequency spectrum for the raw data, Line No. 3. The results show that the central frequency is close to $15 \mathrm{~Hz}$. In the synthetic seismogram, the central frequency was $20 \mathrm{~Hz} . \ldots \ldots \ldots \ldots$

4.15 Average Poisson's ratios for different lithologies. These ratio values were taken into account in the compressional wave and shear wave velocities selection in the synthetic seismograms (Bourbie et

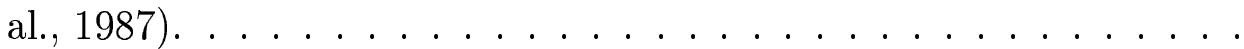

4.16 Synthetic seismogram for Lake Maracaibo obtained using a layer configuration with a mud layer with gassy sediment and the seismic geometry acquisition split spread. The model configuration was composed with 15 layers. . . . . . . . . . . . . 
FIGURE

4.17 Seismic data set from Lake Maracaibo in the mud layer area with gassy sediment and the synthetic seismogram built for this zone. Several similarities can be observed in both situations. . . . . . . .

4.18 Velocity profile used to build the synthetic seismogram in Lake Maracaibo for the mud layer zone, with gassy sediment, but without the rigid shale. Three different kinds of attenuation mechanisms are considered: viscous losses, solid friction losses and gassy sediments effect. . . . . . . . . . . . . . . .

4.19 Synthetic seismogram built for the mud layer with gassy sediment without and with the rigid shale. In this case, it is not possible to appreciate the destructive interference in the Stoneley wave in the synthetic seismogram without the rigid shale layer. . . . . . .

4.20 Synthetic seismogram with the same layer configuration, velocities, thicknesses, densities, etc., but with different $n$ value, in the gassy sediments zone. In the figure on the right, the value for $n$ was 1 ; an the left, the value for $n$ was $0 \ldots \ldots \ldots$ 


\section{CHAPTER I}

\section{INTRODUCTION}

The amplitude of a seismic wave decreases as it propagates because of geometrical spreading, absorption, scattering and other factors associate with the medium through which it travels. As a result of the geometrical spreading, the intensity and the energy density of the spherical waves decrease inversely with the square of the distance from the source.

A plane wave can propagate in an ideal homogeneous, unbounded medium without intensity reduction. The intensity of waves diverging from a real source, however, decreases as the distance from the source increases. This does not represent an energy loss but is simply a consequence of the fact that the constant energy of the wave is distributed over a progressively larger wavefront. Either plane or diverging waves can also experience intensity reduction from other mechanisms. This intensity reduction is called attenuation.

Absorption is defined as any process involving conversion of acoustic energy into heat. This loss is caused by repeated pressure fluctuations in the medium as the wave is propagated. The scattering of a wave propagating in a material medium is a phenomenon in which the direction, frequency or polarization of the wave is changed when the wave encounters discontinuities in the medium, or interacts with the material at the atomic or molecular level. Thus, scattering results in a disordered or random change in the incident energy distribution (Aki and Richards, 1980; Toksöz and Johnston, 1981; Sheriff and Geldart, 1995).

When the mud layer is present in lake floor sediment, several modifications of the lake floor acoustical properties are commonly observed. Compressional wave velocity is reduced below the value for the water-saturated sediment, and consequently, the attenuation of compressional waves propagating through the gassy sediment layer is increased, along with acoustic reflection and scattering from the gassy sediment. The sound speed, scattering strength, attenuation and reflectivity exhibit frequency

This thesis follows the style and format of Geophysics. 
dependent variation. Almost all of these acoustical characteristics are present in the seismic data record from Lake Maracaibo in Venezuela. However, other seismic phenomena are frequently observed in this same location, including decreased resolution as depth increases, hard reverberation or ringing, linear noise, guide waves and mud waves (Levin, 1962).

For several years all these factors were ascribed to the mud layer only. However, recent geotechnical studies show that this problem is more complex than previously thought, because obtaining quality seismic data is not a simple, direct function of location in the lake or thickness of the mud layer (Michelena et al., 1998). For example, in the eastern section of the lake, where the bottom has a thick layer of mud, different reports show low seismic data quality, caused by the low signal/noise ratio and high frequency ringing. However, in the western section of the lake, where the mud layer is very thin, the low seismic data quality is caused by high frequency noise, more than $45 \mathrm{~Hz}$, which damages the seismic data and produces an overlap in the frequency spectrum beyond $45 \mathrm{~Hz}$.

The objective of the present research is to verify if the gassy sediment located in the mud layer generates the low signal/noise ratio observed in the seismis data from Lake Maracaibo. This study will use synthetic seismograms created with different layer configurations designed to simulate the stratigraphic distribution in Lake Maracaibo. Different parameters such as velocity, density, layer thicknesses, and attenuation will be tested. This parameter distribution will be designed to compare two different scenarios.

The first scenario includes the mud layer zone without gassy sediment. In this case, it will be shown that the ringing is the principal phenomenon that can be observed in the seismic data and that three different attenuation mechanisms are present. These are the viscous losses between the particles and the fluid in the suspension above the lake floor, the solid friction losses between the particles in the deeper part of the lake where the concentration of solid particles increases, and attenuation in the mud layer itself that is caused by the same solid friction behavior. Additionally, it will be shown that it is possible to observe a very low shear wave velocity in Lake Marcaibo below $30 \mathrm{~m}$ in depth and that this same layer has a strong attenuation of shear waves' amplitude.

The second scenario examines the mud layer problem, where the mud includes gassy sediment. In this situation, it will be shown that the gassy sediment produced 
the low signal/noise ratio observed in the raw seismic data from Lake Maracaibo. Additionally, it will be demonstrated that at least four different attenuation factors are present in this zone, produced by viscous losses, solid friction, the mud layer without gassy sediment and the mud layer with gassy sediment.

Finally, it will be demonstrated that the most important difference between the area without gassy sediment and the area with gassy sediment can be explained by the existence of an impermeable layer embedded in the mud layer zone that produced high compressional and shear wave velocities. In this way, it will be shown that the attenuation quality factor $Q$, generated for the gassy sediment over the compressional wave, is between 5 and 20, and that the Poisson's ratio is between 0.10 and 0.15 in this zone. Also, it will be demonstrated that this gassy sediment produces high amounts of ringing with low frequency content due to different factors. The Stoneley wave is generated for the combination due to the velocities from the high velocity layer, and the compressional and shear waves velocities in the gassy sediment zone. 


\section{CHAPTER II}

\section{BASIC THEORY}

The attenuation and velocities of seismic waves are two important parameters used to quantify and physically characterize the earth medium for geophysical investigations. Using compiled measurements of attenuation, and intrinsic absorption and scattering loss due to distributed heterogeneties, it is possible to understand the physical mechanisms of attenuation, and attempt to understand the earth composition and structure.

The relationships among frequency, compressional wave velocity and attenuation have important implications for determining parameters for acoustic, elastic or viscoelastic models for unconsolidated sediments, consolidated sediments and gassy sediments.

It is well known that the velocity rises as the frequency increases, in saturated porous media, producing dispersion or dissipation in the body wave velocities. This is explained by the fact that the inertial forces increase simultaneously. This inertial force is associated with the redirection of fluid or gas flow in the porous media. Because these inertial forces are different for the fluid, the solid and the gas part, they generate a differential movement among the fluid, the solid, the gas, the fluid/solid, the gas/solid and the fluid/gas combinations.

In the fluid and fluid/solid case, this implies that the overall movement displacement involves less fluid, whatever type of wave is considered. Hence as the mass involved in overall movement declines progressively with increasing frequency, the velocity increases with frequency. However, the differential movement thus facilitated by increase in frequency causes increasing dissipation. This dissipation is proportional to the square of the angular frequency. For the $P$ and $S$ waves, this means attenuation increases with frequency (Bourbie et al., 1987), and this attenuation, in fluid saturated porous media, is due to the relative motion of the fluid and the solid (Wyllie et al., 1962).

In Lake Maracaibo, several features are observed in the seismic data and cores information. These features suggest that important lateral and vertical changes, in lithology and layer distribution, are observed in the lake in the first $100 \mathrm{~m}$. These 
differences produce changes in the velocity profile as a function of position and mud layer composition. Additionally, these lateral and vertical changes generate different attenuation factors. In this way, unconsolidated, consolidated and gassy sediments are observed in the lake and their distribution controls the velocity profile and the attenuation factor in this zone. Therefore, a brief basic theory about compressional wave velocity, shear wave velocity and attenuation for these three different situations, is described as follows.

\subsection{Compressional and Shear Waves' Speed}

The seismic wave velocity depends on many parameters, especially porosity, saturation, state of stress and pore pressure, temperature, pore fluid type and pore fluid property, lithology, clay content and the frequency of the wave. Furthermore, these parameters often are not independent of each other. The sediments are anisotropic materials whose physical properties vary laterally and in depth, therefore the ideal laws of elasticity cannot be applied.

However, the starting point in any discussion of the propagation of elastic waves in high porosity sediments must be through the expressions for the velocities of the $P$ and $S$ waves, where no relative movement occurs between the fluid and the overall movement or closed system. Equations (2.1) and (2.2) are reference velocities corresponding to the limit velocities of the first kind $P$ wave and the $S$ wave, when frequency tends towards zero:

$$
\begin{gathered}
\alpha=\left(\frac{\lambda+2 \mu}{\rho}\right)^{1 / 2}, \\
\beta=\left(\frac{\mu}{\rho}\right)^{1 / 2},
\end{gathered}
$$

where $\alpha$ and $\beta$ are the compressional and shear velocities respectivebly, $\rho$ is the density, and $\lambda$ and $\mu$ are the Lamé constants. The significance of the shear modulus or rigidity, $\mu$, is readily apparent as a measurement of the material's resistance to shear stress. For a fluid, $\mu=0$, as $\mu$ increases, the body is more rigid. The other Lamé parameter, $\lambda$, is most significant in combination with other terms. Any increase in rigidity above zero, which one might expect in most sediments, would increase the 
value in the numerator of equations (2.1) and (2.2), and thus increase the velocity. It appears, however, that in many high porosity sediments there is little rigidity, and the low $S$ wave velocity predictable by equation $(2.2)$ is present.

Compressional wave velocity is often determined by picking the time of a certain feature of a propagating pulse, such as the first amplitude maximum. However, attenuation changes the shape of a propagating wave, making determination of a physically meaningful velocity problematic. As a consequence, the velocities so determined are not necessarily representative of the material's intrinsic wave phase and group velocities. Another factor in determining compressional wave velocity is that the acoustical properties in unconsolidated or consolidated sediments are significantly different from those of gassy sediments.

\subsubsection{Unconsolidated Sediments}

The relationships between compressional wave speed and several physical properties of unconsolidated sediments, sediments without gas bubbles, have been known for many years (Urick, 1948; Hamilton, 1956; Shumway, 1960; Wood and Weston, 1964).

Porosity is the most important single factor causing variations in compressional wave speed in unconsolidated sediments. This is because the difference in compressibility between water and grains is large, and porosity is a measure of the relative amounts of fluid and solid present. Other important factors that cause variations in compressional wave speed are the rigidity of the sediment, which appears to be related to the abundance of coarse grains; pressure; temperature; the compressibility of the grain aggregate, determined from compressibility of individual minerals; etc.

Differences in densities of the most prevalent mineral grains found in sediments are small, therefore the aggregate sample grain density changes little from sample to sample (Shumway, 1960). The effect of grain density variations are not negligible, however. Grain shape and the spatial interrelationship of grains are important factors, because they determine not only the characteristic of fluid flow past the grains, but also determine, along with grain size, the extent to which a frame incompressibility and rigidity develop between grains. Considering the extremes of porosity (0.40 - 0.85), hydrostatic pressure (0 bars - 1100 bars), temperature $\left(-2^{\circ} \mathrm{C}-35^{\circ} \mathrm{C}\right)$ and mineral composition, which may be present in the marine sediment environment, 
compressional wave speed in unconsolidated sediments, without gas bubbles, is affected by the individual factors described in Table 2.1. The porosity and the rigidity in the sediment are the most important factors causing variations in compressional wave speed in unconsolidated sediments without gas bubbles.

Table 2.1. Relationships between compressional wave speed and physical properties of unconsolidated sediments, without gas bubbles (Shumway, 1960).

\begin{tabular}{|c||c|}
\hline Factor & Change in Sound Speed [\%] \\
\hline \hline Porosity & $15 \%-17 \%$ \\
\hline Rigidity & $9 \%-21 \%$ \\
\hline Pressure & $9 \%-12 \%$ \\
\hline Temperature & $6 \%-8 \%$ \\
\hline Grain aggregate compressibility & $2 \%-4 \%$ \\
\hline
\end{tabular}

Suspensions of small solid particles in liquids retain their fluid feature with no shear strength, up to large concentrations of solid in the mixture. However, in consolidated sediments, an important sediment parameter is the dynamic shear modulus or rigidity, which is not zero. These factors are important to consider in Lake Maracaibo, because the lake contains a very large quantity of solid particles in suspension and consolidated sediments. These features produce a change in the compressional wave speed and increase the compressional wave attenuation. This compressional wave attenuation is produced by different mechanisms: the viscous losses between the particles and the fluid, and the solid friction losses between the particles. This volume of suspended sediment is located between the surface floor and extends less than three meters above the surface floor.

Wood and Weston (1964) proposed equation (2.3) for predicting sound speed in uniform suspensions. Each component contributes to the bulk compressibility and bulk density of the suspension in proportion to its volume concentration in the suspension:

$$
v_{f}=\left(\frac{1}{\left[v_{m} \beta_{m}+\left(1-v_{m}\right) \beta_{w}\right]\left[v_{m} \rho_{m}+\left(1-v_{m}\right) \rho_{w}\right]}\right)^{1 / 2}
$$


where $v_{m}$ is the fraction of the suspension volume occupied by solids, $\rho_{m}$ is the density of the mineral, $\beta_{m}$ is the compressibility of the mineral, $\rho_{w}$ is the density of the water, and $\beta_{w}$ is the compressibility of the water. Experimental confirmation has been published by Urick (1948) and Hampton (1967).

An important feature of equation (2.3) is that the predicted compressional wave speed can be lower in dilute suspensions than in the individual components. The relationship between compressional wave speed and porosity for unconsolidated marine sediments without gas bubbles was estimated by Shumway (1960). Figure 2.1 shows this relationship.

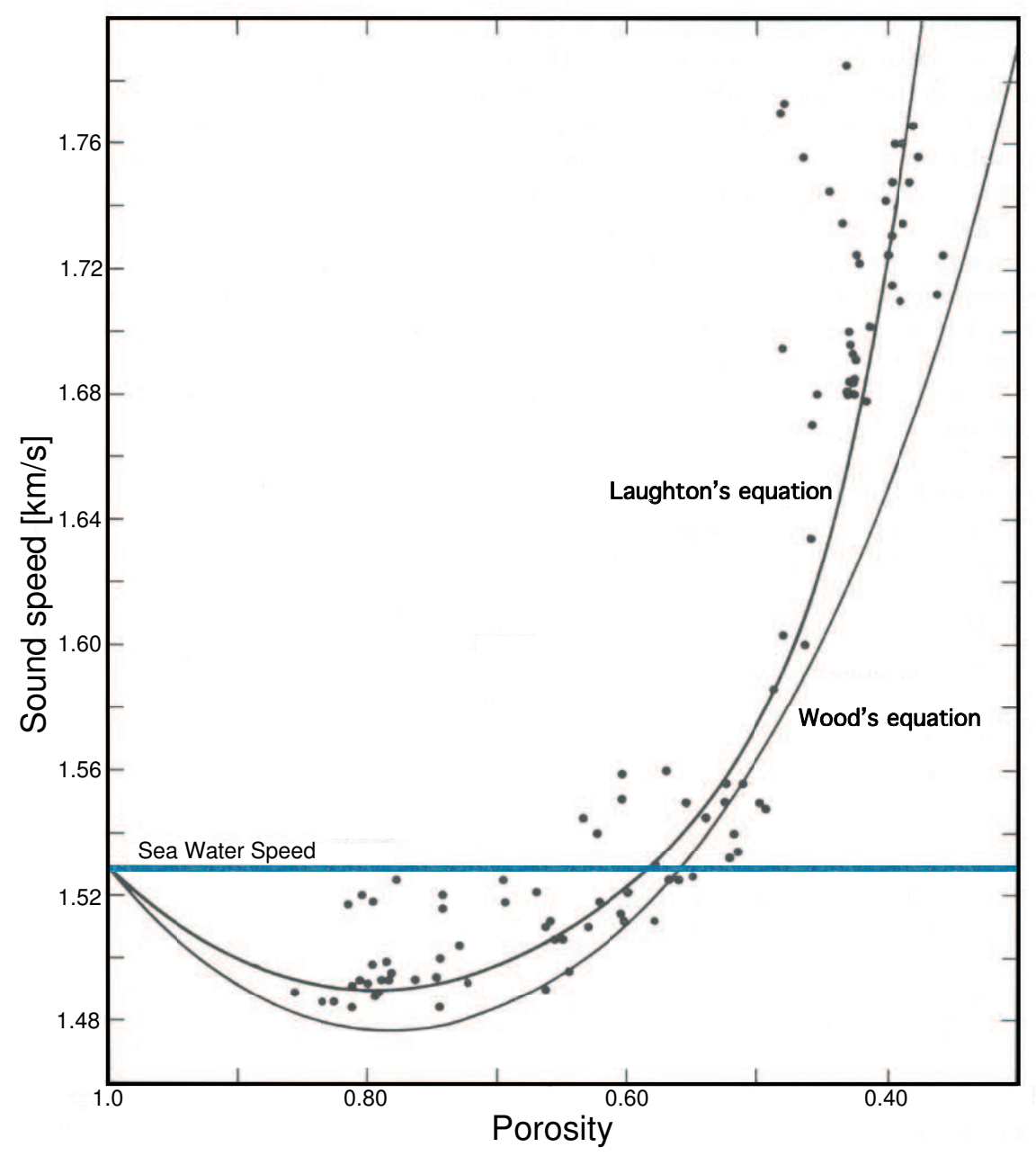

Fig. 2.1. Compressional wave speed versus porosity for unconsolidated marine sediments. Data for 110 samples were used to show the relationship of porosity to compressional wave speed (Shumway, 1960). 
Data for 110 samples were used to show the relationship of porosity to compressional wave speed, shown in Figure 2.1. The mean temperature at which these samples were measured was $23^{\circ} \mathrm{C}$, and the standard deviation of temperatures from this mean was $2.1^{\circ} \mathrm{C}$. The compressional wave speed in sea water of $35 \%$ salinity at this temperature was $1529 \mathrm{~m} / \mathrm{s}$. More than one third of the sediment speed values lie below this water value. Measured speed ranged from $1484 \mathrm{~m} / \mathrm{s}$ to $1785 \mathrm{~m} / \mathrm{s}$. Measured porosity ranged from 0.36 to 0.86 . Results in Figure 2.1 show that although compressional speed increases as porosity decreases, the relationship is not linear.

The theoretical estimation of compressional wave speed using Wood's equation with quartz as the mineral in water can be observed in Figure 2.1. This equation predicts a parabolic curve with a minimum compressional wave speed at a porosity of about 0.80 . This minimum speed is about three percent below the speed in water alone. Shumway (1960) mentions that the curve in Figure 2.1 lies significantly below the measured values of the sands, in the porosity range 0.35 to 0.45 , because rigidity has been neglected. The curve lies significantly below the measured values of clays and finer silts, in the porosity range 0.65 to 0.85 , because the sediment particles consist mainly of clay minerals rather than quartz, and the compressibility of clay minerals is smaller than the value for quartz.

Another important fact about equation (2.3) is that the compressional wave speed variation is similar if other minerals are considered (Hamilton, 1956). Anderson and Hampton (1980a) made a similar estimation using a suspension of kaolinite clay in water. The results are shown in Figure 2.2.

The density ratio of kaolinite to water is 2.71 , whereas the compressibility ratio is 0.02. At low mineral concentrations, the suspension bulk density increases faster than the compressibility decreases, and the next effect is a decrease in the sound speed. At concentrations of about $20 \%$, the suspension bulk compressibility decrease becomes comparable to the density increase. Further increases of mineral concentration decrease the compressibility more than they increase the density, and thus compressional wave speed increases.

For sediments, in general, as median, mean or mode grain size diameter increases, porosity decreases. Shumway (1960) showed that this relationship is approximately linear when grain size is measured in $\phi$ units. The $\phi$ scale, proposed in 1934 by W. C. Krumbein, is a logarithmic transformation of the C. K. Wentworth grade scale based on the negative logarithm to the base 2 of the particle size. Equation (2.4) shows the 


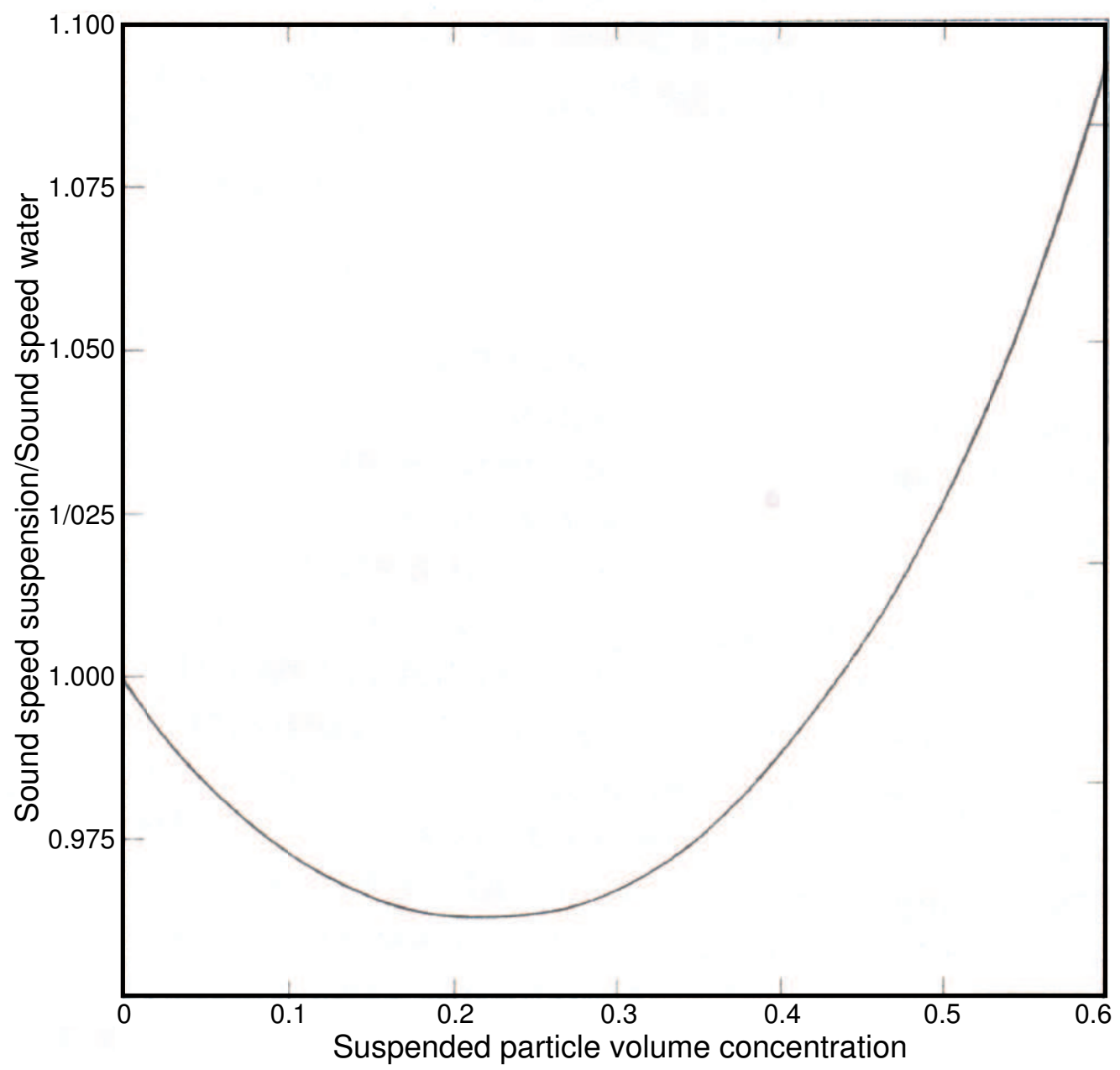

Fig. 2.2. Compressional wave speed versus suspended particle volume concentration. In this case the estimation was made using a suspension of kaolinite clay water (Anderson and Hampton, 1980a). 
relationship between $\phi$ units and the diameter of the particle in $\mathrm{mm}$, where $d_{m m}$ is the diameter of the particle in $\mathrm{mm}$ :

$$
\begin{aligned}
\phi & =-\log _{2} d_{m m}, \\
d_{m m} & =2^{-\phi} .
\end{aligned}
$$

Figure 2.3 shows the relationship between porosity and mean particle diameter for unconsolidated sediments without gas bubbles. The porosity and median diameter relationship has a correlation coefficient of 0.95 .

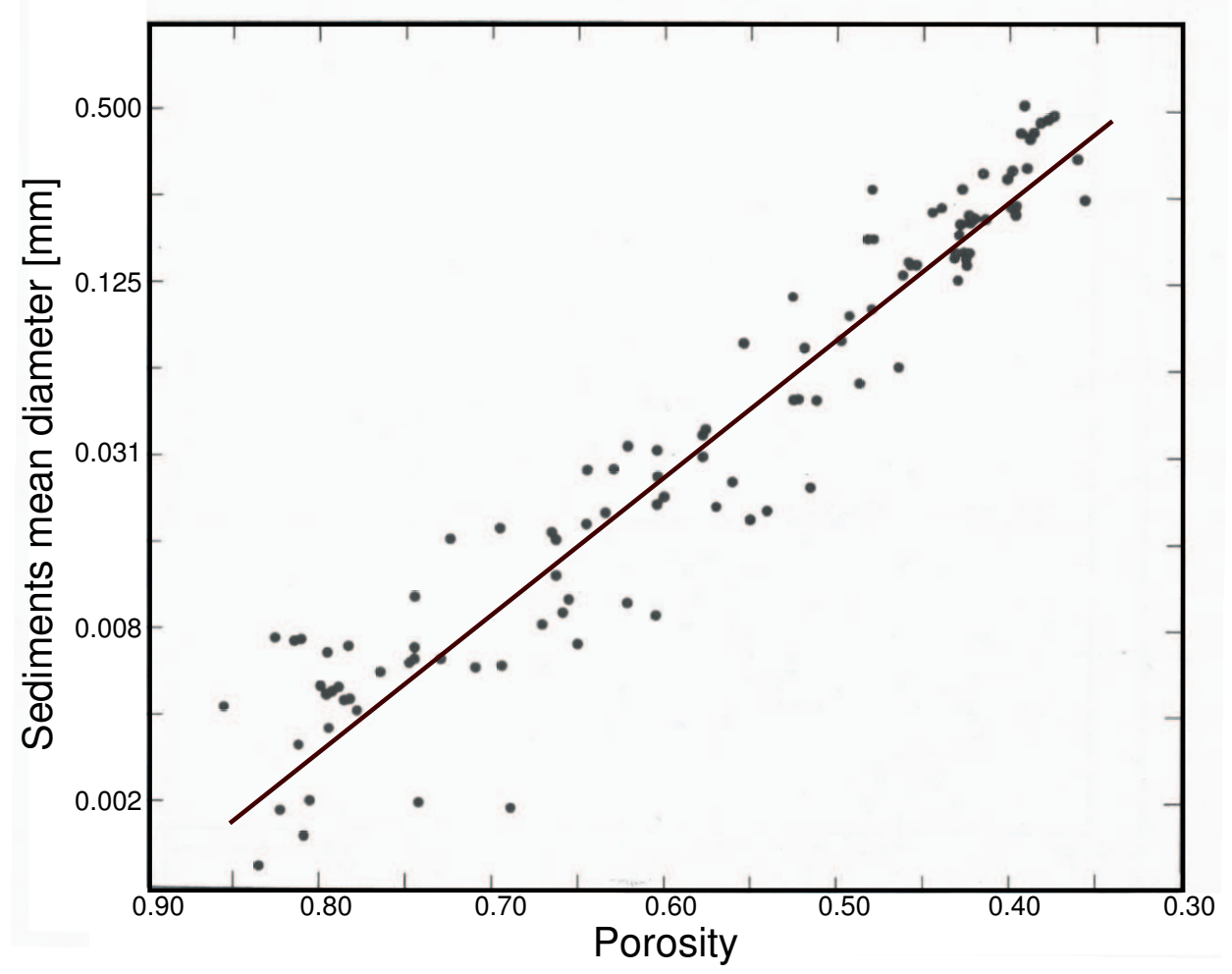

Fig. 2.3. Relationship between mean particle diameter and porosity for unconsolidated marine sediments without gas bubbles. The correlation coefficient is 0.95 (Shumway, 1960).

Shumway (1960) mentions that as a result of this correlation between compressional wave speed and mean grain diameter, a distinct relationship between compressional wave speed and mean grain diameter is found. This can be observed in Figure 2.4. Now, it may be said that, in general, the smaller the median or mean diameter, the lower the sound speed. The relationship is not linear, for it is primarily 
due to the non-linear compressional wave speed and porosity relationship, Figures 2.1 and 2.2. Added to the porosity effect is the effect of the rigidity, which increases as porosity decreases and grain size increases.

The general sediment contains both particles in suspension and particles in contact. The effective rigidity of the sediment, therefore, should be a function of the volume fraction occupied by these particles in contact and their enclosed interstice. In general it is the large particles, that is, the sands and coarser slit particles, which contact one another, while the finer particles, many of which are in the colloidal state, remain effectively in suspension (Shumway, 1960).

\subsubsection{Consolidated Sediments}

For consolidated sediments, equation (2.3) predicts the minimum value of the compressional wave speed. The general function of the compressional wave speeds versus porosity data follows equation (2.3), and the behavior can be observed in Figures 2.1 and 2.2. As shown in Figure 2.1, measurements of sound speed in marine sediments have a compressional wave speed lower than the overlying water. Hamilton (1972) used Wood's equation to explain these low compressional wave speeds. In this case, Hamilton (1972) cautions that most marine sediments exhibit rigidity $(\mu>0)$ and, therefore, would have compressional wave speeds higher than equation (2.3) predicts. The increase in compressional wave speed produced by the sediment shear rigidity is the maximum for fine sands, and is the minimum for clays. The finite shear modulus in marine sediments is the result of a framework or skeleton formed within the sediment. As interparticle forces come into effect, either via grain to grain contact in sands or via electrostatic and London-Van Der Waals forces in slits and clays, an elastic framework is formed that modifies the elastic properties of the aggregate. This elastic framework raises the shear modulus above zero and also increases the bulk modulus.

Anderson and Hampton (1980a) explain several considerations for determining the influence of frame bulk modulus. This research shows that frame bulk modulus is small for clays, and becomes increasingly significant as the sediment particle size increases or the porosity decreases. In several studies, the shear bulk modulus and bulk modulus are considered together, because their effects are difficult to separate in consolidated media. These two factors for a given sediment increase as it consolidates, 


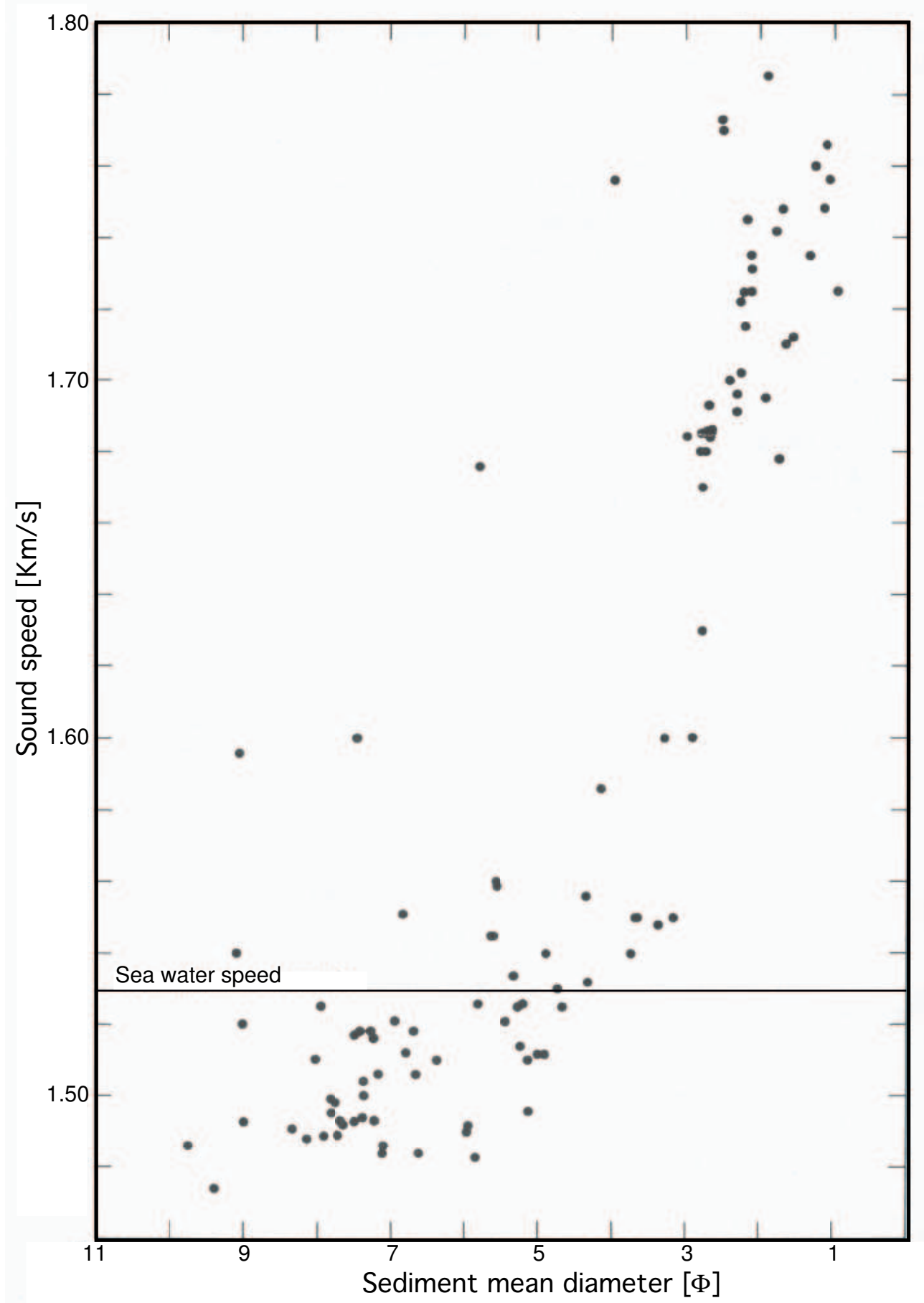

Fig. 2.4. Relationship between sediment particle diameter and compressional wave speed for unconsolidated marine sediments without gas bubbles (Shumway, 1960). 
loses water and develops intergranular cementation. These changes take place as sediment ages and becomes buried under younger materials.

Two additional parameters affecting compressional wave speed in consolidated sediments are temperature and pressure. Shumway (1960) showed that compressional wave speed in sediments is related to temperature in the same way that compressional wave speed in sea water is related to temperature. This research shows that the effect of temperature on compressional wave speed is considerable, amounting to an increase of $10 \%$ to $15 \%$ in going from $\mathrm{O}^{\circ} \mathrm{C}$ to $60^{\circ} \mathrm{C}$. Shumway (1960) attributes this to the important role of sea water compressibility in the composite compressibility of the sediment and to the fact that the change of sea water compressibility with temperature is large compared to the change of mineral compressibility with temperature. In this way, it is possible to infer that the dynamic shear modulus and dynamic frame bulk modulus must also be relatively insensitive to temperature. However, in Lake Maracaibo the effect of temperature is less important than in sea water depth, because the maximum depth in Lake Maracaibo is $50 \mathrm{~m}$.

There are several pressures which must be considered when ascertaining the dependence of the compressional wave speed in consolidated sediments on pressure. Anderson and Hampton (1980a) mention that the pore fluid pressure and the frame pressure are of interest. For superficial sediments, pore fluid pressure will be equal to the hydrostatic pressure in the sea water. Frame pressure is due to the buoyed weight of mineral grains. Therefore, the frame pressure is zero at the water/sediment interface. Compressional wave speed in consolidated sediments is affected by overlying water depth through changes of the sea water bulk modulus with temperature and pressure (Shumway, 1960).

As sediments are buried by additional sediments, the frame pressure increases due to the weight of the added material. This causes a decrease of porosity. Because of compression of the frame, the pore pressure also increases, but pressure gradually decreases as pore water is forced out of the interstitial spaces of sediment. The net effect of these actions is a decrease of porosity and an increase of bulk density with depth burial. Anderson and Hampton (1980a) mention that contraction of the sediment frame may also result in increased dynamic shear modulus and dynamic frame bulk modulus. In samples of clay and mud, compressional wave speed increases with increasing consolidation pressure. This agrees with observations of marine sediments in-situ. 


\subsubsection{Gassy Sediments}

It is well known that the presence of a small volume of gassy sediment can greatly alter the compressional wave speed and increase the attenuation factor of the acoustic wave as the wave is traveling through this medium. The reduction of the compressional wave speed in gassy sediment is similar to the reduced compressional wave speed for propagation through a cloud of gas bubbles in water (Anderson and Bryant, 1990). For low frequency compressional waves, the composite compressibility of the gas bubble/water mixture is essentially reduced to the compressibility of the gas alone. This low bulk compressibility results in the low propagation speed of the compressional waves through the mixture. Similar behavior of gas in sea floor sediments is observed for low frequency compressional wave propagation and for sediments with low shear strength. As sediment shear strength increases, the bulk compressibility effects of gas bubbles in the sediment are reduced.

An expression similar to equation (2.3) can be written for the compressional wave speed in gassy sediment. Anderson and Hampton (1980a) mention that this is possible if each component contributes to the sediment bulk compressibility and bulk density in proportion to its fractional volume concentration in the mixture. The compressional wave speed can be estimated in several steps. First, the density is given by:

$$
\rho=n_{g} \rho_{g}+(1-n) \rho_{m}+\left(n-n_{g}\right) \rho_{w},
$$

where $\rho$ is the aggregate bulk density, $\rho_{g}$ is the density of the gas, $\rho_{m}$ is the density of mineral particles, $\rho_{w}$ is the density of interstitial water, and $n$ is defined as the fractional porosity. The gas porosity, $n_{g}$, is equal to gas volume over the total sediment volume. Next, the bulk modulus of the interstitial fluid, containing both water and gas, is computed as

$$
K_{w}^{o}=\frac{K_{w} \gamma P_{o}}{n_{g}^{o} K_{w}+\left(1-n_{g}^{o} \gamma P_{o}\right)} .
$$

Here $K_{w}^{o}$ is the bulk modulus of the interstitial water modified by gas, $K_{w}$ is the unmodified bulk modulus of interstitial water, and $\gamma$ is the ratio of specific heats of gas. $\gamma$ is related to the period of oscillation, the mass, the diameter of the gas bubble, the volume and the pressure. $P_{o}$ is defined as the ambient hydrostatic pressure and $n_{g}^{o}$ is is the fraction of the sediment pore space occupied by gas. If the $n_{g}^{o}$ is expressed as 
a percentage, then this is equal to $100 \%$ minus the water saturation of the sediment. The modified water bulk modulus, $Q^{o}$, due to changes in the compressibility of the gas in the sediments is:

$$
Q^{o}=K_{w}^{o}\left[\frac{K_{m}-K_{f}}{n\left(K_{m}-K_{w}^{o}\right)}\right]
$$

where $K_{w}^{o}$ is the modified water bulk modulus defined in equation (2.6), $n$ is the sediment fractional porosity, $K_{m}$ is the mineral particle bulk modulus and $K_{f}$ is the sediment frame bulk modulus. The final expression for the aggregate bulk modulus of the sediment sample is then:

$$
K=K_{m}\left[\frac{\left(K_{f}+Q^{o}\right)}{\left(K_{m}+Q^{o}\right)}\right] .
$$

Given these preceding results, the expression to estimate the compressional wave speed in gassy sediment is then:

$$
\alpha_{g}=\left(\frac{K+\frac{4}{3} \mu}{\rho}\right)^{1 / 2},
$$

where $\rho$ is the bulk density of the mixture of sediment particles, water and gas given by (2.5), and $K$ was defined in equation (2.8). This equation can be used to estimate the compressional wave speed in gassy sediment with either large or small gas content, because the shear rigidity $\mu$ and frame bulk modulus $K_{f}$ were included in the expression, and the shear rigidity and the frame bulk modulus will modify the compressional wave speed in gassy sediment medium (Anderson and Hampton, 1980b).

Large gas content will cause sediment expansion, and therefore reduction of grain contacts and reduced strength. This can cause sediments to collapse. Equation (2.9) assumes that the greater compressibility of gas increases the interstitial water compressibility, changing the mineral compressibility, frame compressibility or shear rigidity.

Anderson and Hampton (1980b) estimated the compressional wave velocity with four different sediments, using equation (2.9). The parameters used to compute the compressional wave speed were obtained from previous works (Anderson and Hampton, 1980a). Figure 2.5 shows the estimation of the compressional wave velocity in gassy sediment. The decrease in total sediment bulk modulus is greatest in clay and least in coarse sand. In all of the sediment types, for low gas content, the compres- 
sional wave speed decreases with increasing gas content, until the gas content exceeds $1 \%$ of pore space, where the decrease in density causes a small increase in sound speed with increasing gas content. This compressional wave velocity represents the upper bounds in the velocity estimation, because equation (2.9) assumes that structural strength is unmodified by the addition of gas to a saturated sediment.

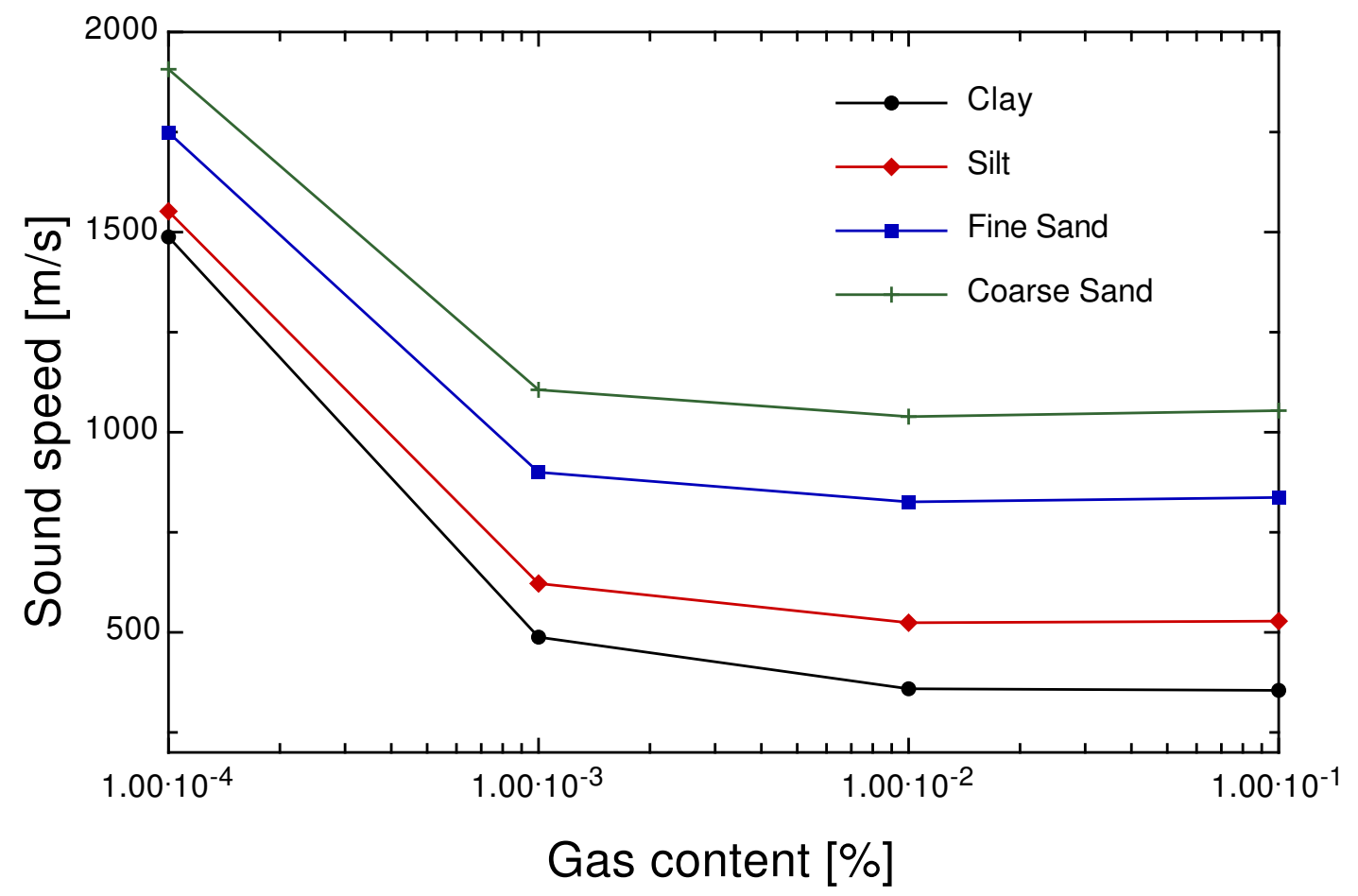

Fig. 2.5. Compressional wave speed versus gas content, in percentage, using one atmosphere pressure. The porosity was between 0.35 to 0.85 . The frame elastic properties were included (Anderson and Hampton, 1980b).

Equation (2.9) should be used for estimating the compressional wave speed in gassy sediment for frequencies less than the fundamental resonance frequency of the bubbles. Three distinct frequency ranges can be identified, each with different acoustical behavior: frequencies below the fundamental resonance, frequencies in the vicinity of the fundamental resonance and frequencies above the fundamental resonance.

The fundamental resonance frequency is defined as the frequency match between the natural frequency of the system and the external forced vibration frequency. If the frequency of a period force applied to a body is the same as, or very near to, its natural frequency, that body will vibrate with very large amplitude due to the force. At 
frequencies below the bubbles resonance frequency, the phase velocity is substantially decreased by the presence of the bubbles. Near to the bubble resonance frequency, the attenuation increases markedly; but above the bubble resonance frequency, the phase velocity and the attenuation approach the values which they would have if no bubbles were present.

Anderson and Hampton (1980b) propose an expression for the compressional wave speed at and above the bubble resonance frequency. This equation was used to calculate compressional wave speeds for an ocean bottom slit sediment and for a harbor mud sediment. Figure 2.6 shows the result obtained. In this case, the bubbles resonance effects are much less apparent in the ocean slit sediment. This is because the bubble vibration is damped by the ocean bottom sediment internal friction. For both sediments, the compressional wave speed increases with frequency near resonance. Above resonance, the compressional wave velocity first exceeds, then approaches the values in sediments without gas bubbles.

In Figure 2.6, $c_{o}$ represents the compressional wave velocity in the saturated sediment, $c$ is the compressional wave speed in gassy sediment and $f_{o}$ is the fundamental resonance frequency. It is evident from the equation analysis used to estimate the compressional wave velocity in gassy sediments that the sediment compressional wave speed should be approximately equal to the saturated sediment value for frequencies somewhat higher than the resonance frequency. Therefore, the exact shape of the curve above resonance is a function of gas volume concentration.

Several measurements have been made and reported by researchers confirming that the gas volume concentration must be known to predict the compressional wave velocity in a gassy sediment. Additionally, for frequencies below resonance, a $3 \%$ volume concentration of bubbles will produce compressional wave speed below the consolidated sediment value (Anderson and Hampton, 1980b).

\subsection{Attenuation of Compressional and Shear Waves}

Is well known that the amplitudes of the seismic data depend on different factors such as source strength and coupling, random noise, spherical divergence, reflection coefficient, attenuation by scattering and/or absorption in sediments without gas bubbles, viscous losses between the particles and the fluid, solid friction losses between the particles, frame friction absorption associated with bubble motion, scattering by 


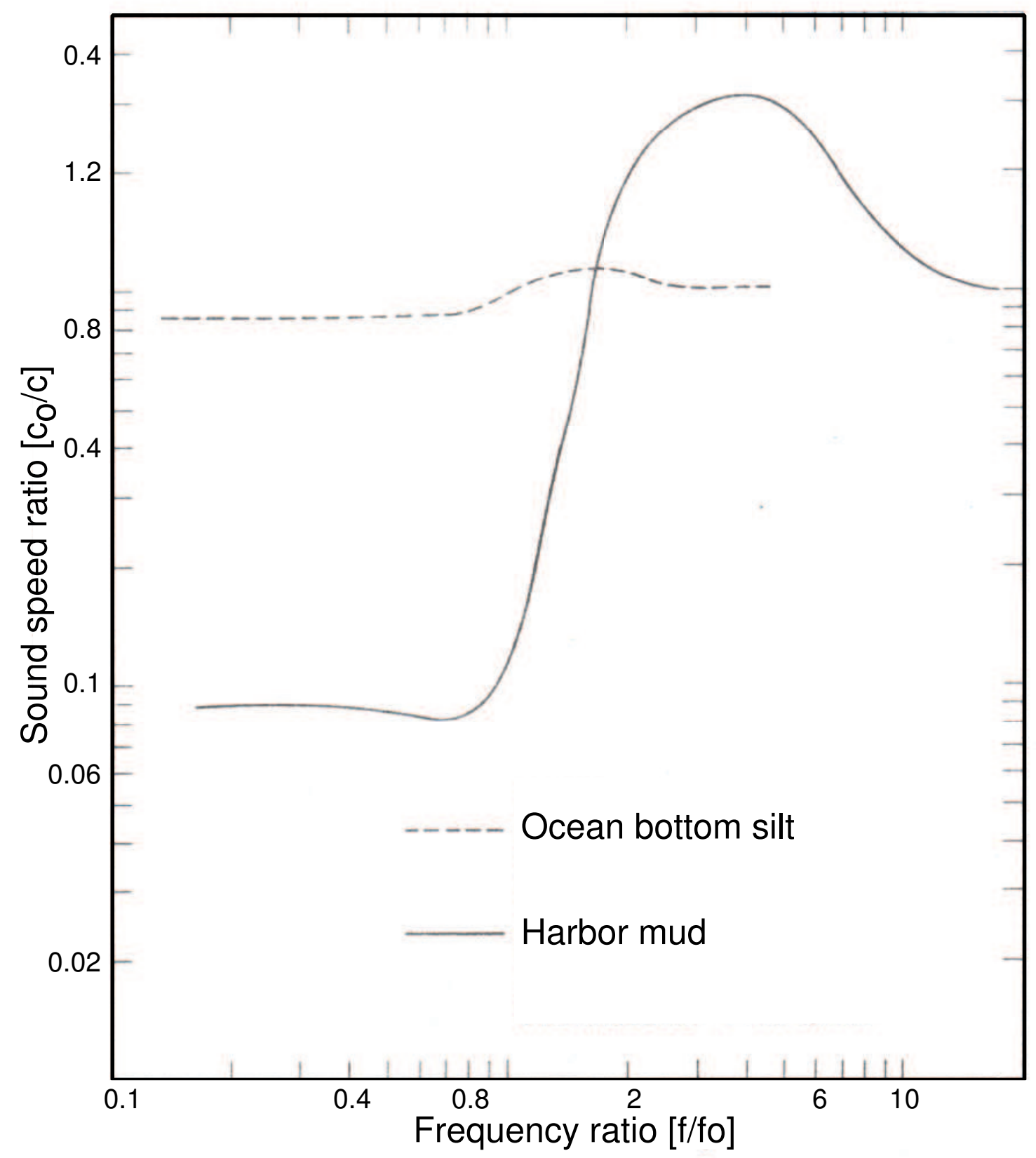

Fig. 2.6. Compressional wave speed ratio in gassy sediment versus frequency ratio. Ocean bottom silt sediment and harbor mud sediment were used to calculate compressional wave velocity (Anderson and Hampton, 1980b). 
bubbles, etc. Several of these factors can be controlled. The effects of others can be determined or estimated, and then compensated for. However, the effect of other factors cannot be estimated, and therefore cannot be compensated for in seismic data acquisition. A wave can experience intensity reduction because of attenuation, and this causes a reduction in amplitude or energy, due to the physical characteristics of the transmitting media. This implies that the amplitude decreases when the distance from the source increases.

The attenuation is a decrease in seismic signal amplitude as waves propagate from the seismic source. Different factors can produce the attenuation in the compressional wave, but the scattering and the absorption are the most important ones to consider. If the anomalous region is small relative to the compressional wavelength or is large and irregular, the intercepted energy will be re-radiated in different directions from the propagation direction of the primary wave, and the wave will be scattered. If scattering centers are distributed throughout a medium, a compressional wave is attenuated as it propagates.

In addition, energy can also be removed from a propagating compressional wave by absorption. There are many absorption conditions, but all of them remove energy, which is converted into heat while passing through a medium. This means that the energy associated with the wave motion is gradually absorbed by the medium, and this process along with others is responsible for the eventual disappearance of the wave motion. This absorption varies with the frequency. This decrease of the amplitude is exponentially related to the distance. The difference between the absorption and the scattering is that the losses by scattering are more important than the losses by absorption for low frequencies and short distances from the sources. As the frequency and the distance increase, absorption losses increase and eventually become dominant (Sheriff and Geldart, 1995).

In the above discussion of the attenuation, two principal attenuation conditions were identified: the irregular and diffuse dispersion of energy by scattering and the energy conversion into heat by absorption. However in sediments without gas, other conditions contribute to compressional wave attenuation. These include viscous losses between the particles and the fluid, and the solid friction losses between the particles (McCann and McCann, 1969; Gibson and Toksöz, 1989). This is important to consider because the velocity in consolidated sediment is less than the velocity of sound in the water just above the bottom. Hamilton (1956) showed that the different is 
between $2 \%$ and $3 \%$ and this change in the velocity represents, in the general case, an increase in the wave attenuation.

The attenuation in gassy sediments is caused by the frame friction absorption associated with bubble motion and scattering by bubbles. Anderson and Hampton (1980a) showed that the attenuation can be very high in gassy sediments for frequencies of resonance. If a distribution of bubble sizes is present, the attenuation will be large over a greater range of frequencies. In this way, a small quantity of gas that produces changes of compressional wave speed can significantly increase the attenuation.

The lake floor, along with the following factors, affects underwater compressional waves: forward scattering; reflection loss and absorption; interference and frequency effects; attenuation for unconsolidated, consolidated or gassy sediments; noise generation at lower frequency due to seismic activity and other operations; back scattering and bottom reverberation, etc. Although the sea floor affects the compressional wave in ways similar to that of the sea surface, the return of the compressional wave from the bottom is more complex than from the surface for several reasons:

- The bottom is more variable in composition.

- The bottom is often stratified with density, compressional and shear wave speeds varying gradually or abruptly with depth.

- The bottom characteristic, composition and roughness can vary over relatively short horizontal distances.

- The compressional wave can propagate through a sedimentary layer, and either be reflected back into the water by sub-bottom layers or be refracted back by the large compressional and shear wave speed gradients in the sediments.

These mechanisms can be incorporated into mathematical models through the specification of appropriate boundary conditions. The complexity of these boundary conditions depends on the level of known detail concerning the composition and structure of the sea floor. Additionally, acoustic interactions with highly variable sea bottom compositions necessitate the inclusion of both compressional and shear effects, particularly at lower frequencies. This is especially important for high concentrations of unconsolidated sediments, consolidated sediments and gassy sediments. 
The most commonly used measures of attenuation are: the attenuation coefficient $\alpha$, which is the exponential decay constant of the amplitude of a plane wave traveling in a homogeneous medium; the logarithmic decrement $\delta$; and the quality factor $\mathrm{Q}^{-1}$, which is the reciprocal specific dissipation function (Toksöz and Johnston, 1981). These values are related by the following expressions:

$$
\frac{1}{Q}=\frac{\alpha v}{\pi f}=\frac{\delta}{\pi}
$$

where $v$ is the velocity and $f$ is the frequency. Both the velocity and attenuation are associated with a particular mode of wave propagation. In equation (2.10), for a lossy material, a second-order term must be included, but under the low loss assumption this term is insignificant and may be dropped. The quality factor $Q$ is the most common measure of the attenuation and it is an intrinsic property of the rock. $Q$ is defined as the ratio of stored energy to dissipated energy. Now, if a volume of material is cycled in stress at a frequency $\omega$, then a dimensionless measure of the internal friction is:

$$
\frac{1}{Q_{(\omega)}}=\frac{\Delta E}{2 \pi E}=\frac{2 \pi W}{\Delta W},
$$

where $E$ is the peak strain energy stored in the volume and $\Delta \mathrm{E}$ is the energy lost in each cycle due to imperfections in the elasticity of the material. $W$ is the elastic energy stored at maximum stress and strain, and $\Delta W$ is the energy loss, per cycle, of a harmonic excitation. Toksöz and Johnston (1981) mention that the quality factor $Q$ may differ under some conditions from $Q$ values derived from processes such as wave propagation. Aki and Richards (1980) mention that the definition shown in equation (2.11) is rarely of direct use, because only in special experiments is it possible to drive a material element with stress waves of unchanging amplitude and period. However, it is possible to observe the following situations:

- The temporal decay of amplitude in a standing wave at fixed wavenumber.

- The spatial decay in a propagating wave at fixed frequency.

Now, with the assumption that the attenuation is approximately a linear phenomenon almost linearly dependent on the frequency, it is possible to apply the Fourier analysis to the attenuation problem and obtain the correct effect of attenuation on actual seismic signals. For a medium with a linear stress-strain relationship 
and using either the first or second above statements, the amplitude $A$ is proportional to the square root of the energy, $\mathrm{E}^{1 / 2}$, where the value of $Q$ is assumed to be larger than one, $\mathrm{Q} \gg 1$, allowing that successive peaks have almost the same strain energy. In this case, the amplitude $A$ may represent a maximum particle velocity, or a stress component in the wave. For this reason, it is possible to obtain equation (2.12):

$$
\frac{1}{Q_{(w)}}=\frac{1}{\pi} \frac{\Delta A}{A}
$$

With equation (2.12) the amplitude fluctuations due to attenuation can be obtained. Thus for the two different situations, temporal and spatial decay, the equations can be written as follows:

$$
\begin{aligned}
& A(t)=A_{o} \exp \left[\frac{w t}{2 Q}\right], \\
& A(x)=A_{o} \exp \left[\frac{w x}{2 Q c}\right],
\end{aligned}
$$

where $A(t)$ and $A(x)$ are the amplitudes of the seismic signal of frequency $w$, after traveling for a length of time $t$ and distance $x$, respectively. $A_{o}$ is the starting amplitude, $w$ is the angular frequency, $c$ is phase velocity, $x$ is the spatial dimension and $t$ is the temporal dimension.

Any spatial decay due to geometrical spreading effects and geometrical dispersion due to material heterogeneity must be considered, because this geometrical dispersion involves differences between the quality factor $Q$ measured using equation (2.13) and $Q$ estimated by using equation (2.14) (Aki and Richards, 1980).

A variety of sediment attenuation units are commonly used, and the relationship among these units can become very confusing when attempting to specify values for propagation models. In general, the mathematical acoustic attenuation spatial expression is described in equation (2.14). The acoustic attenuation $\alpha$ can have units of nepers per unit distance. This acoustic attenuation can be converted to the units of $d B$ per meters using the relationship described in equation (2.15):

$$
\left(\frac{d B}{m}\right)=20(\log e)\left(\frac{n e p e r}{m}\right) \text {. }
$$


Attenuation can be measured in a variety of ways, including stress strain phase lag and dynamic methods using compressional and shear waves (Toksöz and Johnston, 1981). Different $Q$ values are obtained for different methods. In this way, the use of extensional stress strain phase lag results in loss parameter $Q_{e}^{-1}$, that is, the anelasticity of Young's modulus. The bulk loss $Q_{k}^{-1}$ can be obtained from hydrostatic stress and dilational strain. Compressional and shear waves provide values for $Q_{p}^{-1}$ and $Q_{s}^{-1}$. These quantities can be related to one another using the definition of $Q$ given in equation (2.16):

$$
\frac{1}{Q}=\frac{M_{I}}{M_{R}}=\tan \Phi
$$

The above equation establishes that for a nearly elastic or low loss linear solid, an alternative quality factor definition may be found from the stress strain relations (Toksöz and Johnston, 1981). Given sinusoidally varying stress relations, the strain response will also be sinusoidal. These two factors are related through equation (2.16), where $M_{I}$ and $M_{R}$ are the imaginary and real parts of the elastic modulus $M$ relating stress and strain, and the phase lag $\Phi$ of the strain caused by the stress.

Equation (2.16) results in an elliptical stress strain curve under sinusoidal loading. Toksöz and Johnston (1981) mention that the decomposition of $Q$ values into pure compressional and shear losses has proved to be a useful diagnostic aid in the analysis of attenuation mechanisms. Equation (2.16) involves only the complex modulus and it is dimensionless. Because the imaginary part of the modulus is zero for a nondissipative elastic medium, the quality factor $Q$ is infinite. By contrast, a zero quality factor implies an infinitely attenuated medium, without any transmission quality. The equivalence of the definitions of $Q$ between equations (2.12) and (2.16) arises from the fact that the area of the ellipse is proportional to the energy loss per cycle, and the energy stored is given by the total area under the loading curve. Another term commonly encountered in acoustic modeling is the attenuation coefficient $\alpha$, which is based on the concept that attenuation and frequency are related by a power law. Equation (2.17) shows this relationship:

$$
\alpha=k f^{n}
$$

where $\alpha$ is attenuation of compressional waves in $\mathrm{db} / \mathrm{m}, k$ is a constant for a particular 
sediment and is related to porosity and mean grain diameter, $f$ is the frequency dependence in $\mathrm{kHz}$ and gives the dependence of the attenuation upon the frequency. Typically, $n$ is assumed to be unity; but in the build of the synthetic seismogram, the value of $n$ was set between 0 and 1 . However, several experiments show that this value can be between 2 and 3 also (Hamilton, 1972).

\subsubsection{Unconsolidated Sediments}

The attenuation of the compressional waves in unconsolidated sediments is caused by rigid, incompressible particles that are free to move when subjected to pressure fluctuations by a passing wave. The solid particles in liquids retain their fluid character, without shear strength. When the compressional wave passes through groups of touching particles, the particles move and rub against each other. Losses are due to friction. The loss is proportional to particle displacement and does not depend on the particle velocity. The rigidity of the unconsolidated sediments depends on porosity. Because the rigidity depends on particle contact, the friction losses will be larger when the rigidity increases. The attenuation loss in the unconsolidated sediments reduces the reflection coefficient for signals reflected at the water/sediment interface, relative to the no loss case. Beyond the critical angle, the loss causes a reduction of the reflected energy because the compressional wave penetrated the sediments in the process of total reflection. Figure 2.7 shows a compilation of sediment attenuation measurements made by Hamilton (1980) over a wide frequency range. The attenuation in natural, saturated sediments and sedimentary strata is nearly proportional to the frequency.

There was a tendency for more dense sediments, such as compact sands, to have a higher attenuation than the less dense, high porosity sediments, such as muds. In this way, the attenuation decreases with increasing concentration, and this due to increasing transmission of energy through the suspended particles.

Urick (1948) shows, using equations (2.18) and (2.19), that the acoustic intensity attenuation coefficient, $\alpha$, in unconsolidated sediments of small spherical mineral particles of a single size, is obtained by finding the average rate at which work is being done over a large spherical surface surrounding the $n$ particles per unit volume:

$$
s_{1}=\frac{9}{4} r_{m}\left(\frac{2 \zeta}{\omega}\right)^{1 / 2}\left[1+\left(\frac{2 \zeta}{\omega}\right)^{1 / 2}\right],
$$




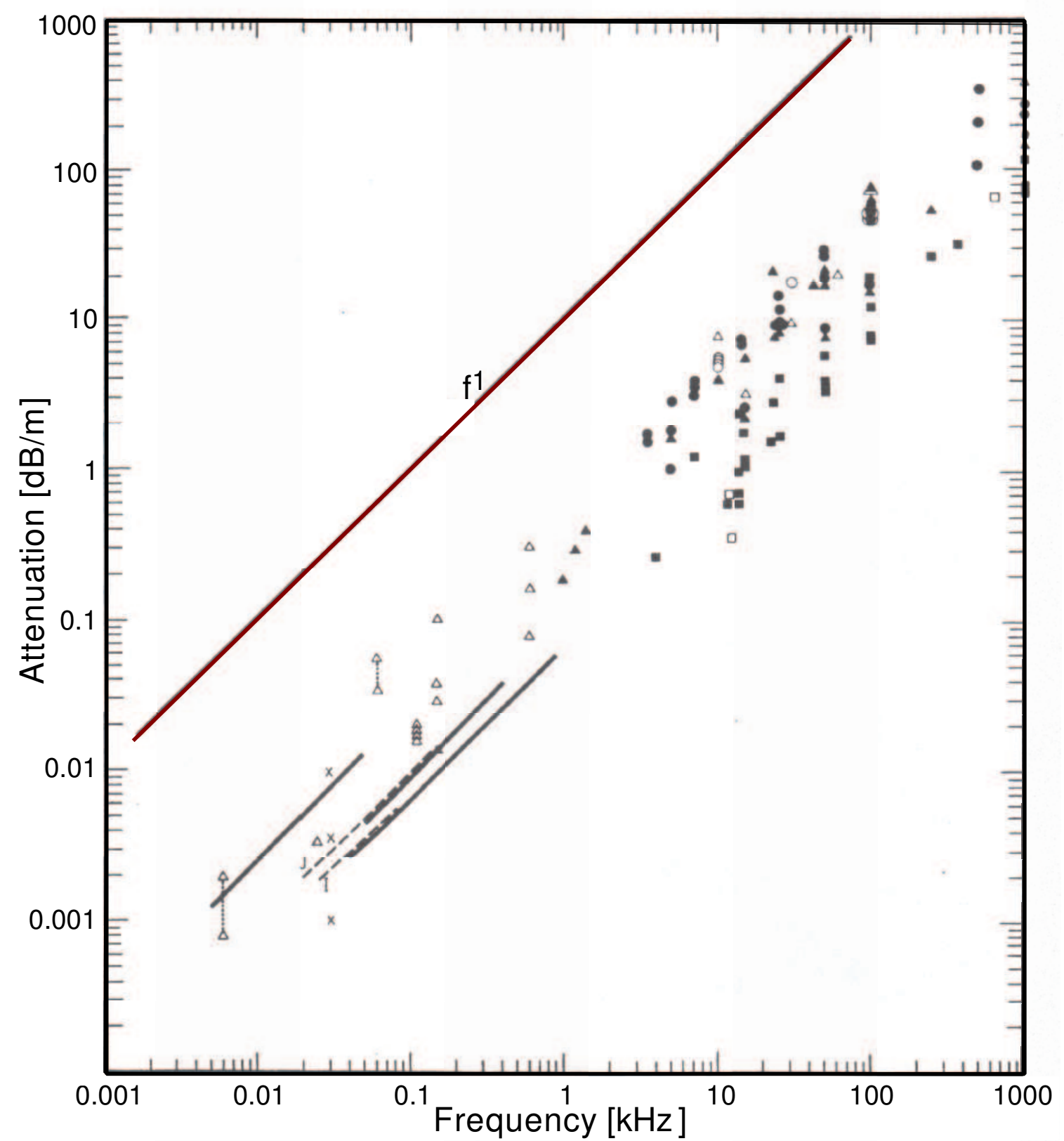

Fig. 2.7. Attenuation of compressional waves versus frequency in natural, saturated sediments and sedimentary strata. The line labeled $f^{1}$ indicates the slope of any line representing a first power dependence of attenuation on frequency (Hamilton, 1980). 


$$
\tau=\frac{1}{2}+\frac{9}{4} r_{m}\left(\frac{2 \zeta}{\omega}\right)^{1 / 2}
$$

where $\zeta$ is defined as the kinematic viscosity of water, $\omega$ is the angular frequency and $r_{m}$ is the radius of mineral spheres. Now, as a function of the above two equations, it is possible to write an expression for the acoustic intensity attenuation coefficient as follows:

$$
2 \alpha=v_{m}\left[\frac{1}{3} k^{4} r_{m}^{3}+k\left(\frac{\rho_{m}}{\rho_{w}}-1\right)^{2} \frac{s_{1}}{s_{1}^{2}+\left[\left(\frac{\rho_{m}}{\rho_{w}}\right)+\tau\right]^{2}}\right],
$$

where $\alpha$ is the acoustic intensity attenuation coefficient, $v_{m}$ is the fraction of the suspension volume occupied by solid, $k$ is the wavenumber, $\rho_{m}$ is the density of the mineral, $\rho_{w}$ is the density of the water, and $s_{1}$ and $\tau$ were defined in equations (2.18) and (2.19) respectively.

In equation (2.20) the first of the two terms on the right is the scattering of acoustic energy by suspended particles, or in other words, the scattering loss produced by small rigid spherical particles free to move in the acoustic medium. This represents a distribution rather than a dissipation of the energy of the primary beam (Urick, 1948).

The second term is a frictional loss or absorption due to the viscosity of the suspending fluid. This absorption is because particles in a sound field partake of the motion of the fluid to an extent determined by their mass, the frequency of the compressional wave and viscosity of the fluid. There will thus exist a velocity difference between fluid and particles, which for a viscous fluid results in a heat loss at the surface of each particle, and therefore a decrease in intensity of the acoustic medium.

Anderson and Hampton (1980a) mention that equation (2.20) assumes multiple interactions between the acoustic energy and the suspended particles, and predicts the viscous absorption will dominate for small values of $k r_{m}$ at low frequencies, whereas scattering will dominate for large values of $k r_{m}$. It also predicts a linear relationship between attenuation and concentration, and this relationship is shown in Figure 2.7. However, McCann and McCann (1969) mention that equation (2.20) shows a linear relationship between concentrations and compressional wave attenuation, when the particle concentration, in medium, is up to $5 \%$. Above this concentration, the linear 
relationship breaks down. The attenuation reaches a maximum at about $20 \%$ concentration and then decreases with further increases in the volume concentration of particles. In Lake Maracaibo the particle concentrations are larger than $5 \%$ and, in this case, it is possible to use equation (2.20).

\subsubsection{Consolidated Sediments}

The features of waves propagating in a consolidated sediment medium depend on the mechanical properties of the fluid, the discrete particles, and the skeletal frame formed by the assemblage of particles. Motion of fluid relative to the frame and the relative motion of contiguous particles are two potential sources of energy dissipation in this kind of medium. Basically, two mechanisms can explain the attenuation of the compressional and shear waves in unconsolidated sediments or consolidated sediments. These mechanisms are viscous losses between the particles and the fluid, and the solid friction losses between the particles (McCann and McCann, 1969).

The solid friction losses occur at the points of contact between the particles. McCann and McCann (1969) mention that it has been established that these losses are identical in nature with the losses in polycrystalline rocks. The solid friction losses are characterized by a linear variation of the attenuation coefficient to frequency and small velocity dispersion, over a frequency range from $1 \mathrm{~Hz}$ to $1000 \mathrm{~Hz}$, and more. The viscous losses of the compressional wave arise because of the acoustic velocity differential between the solid particles and the fluid in the sediment (Gibson and Toksöz, 1989).

For consolidated sediments, the studies and discussions of the dependence of the compressional wave attenuation on frequency must distinguish between sediment types. In mud and similar sediments, such as mud-stone, clay-stone and shale, both theory and experimental evidence indicate an approximate first order dependence of attenuation on frequency, from very low frequency, a few $\mathrm{Hz}$, to high frequency, a MHz. Stoll and Bryan (1969) show several results where it is feasible to obtain reasonable estimates for attenuation over a wide range of frequencies using the Biot's Theory for porous saturated media.

The theory predicts that attenuation is controlled by the solid friction losses at low frequencies, resulting in a linear dependence on frequency, whereas frequency dependent viscous losses dominate at the higher frequencies, producing a dependence 
on $f^{n}$, where $n$ first increases within the range of 1 to 2 and then decreases. Additionally, Stoll and Bryan (1969) found that the logarithmic decrement of shear waves in a consolidated sediment silt to be independent of frequency for low amplitude waves at frequencies between $43 \mathrm{~Hz}$ and $391 \mathrm{~Hz}$. This also indicates a first order dependence of attenuation on frequency for shear waves.

In a study of attenuation in consolidated sands, poorly sorted, in the frequency range from $1 \mathrm{~Hz}$ to $1000 \mathrm{~Hz}$, Hamilton (1980) mentions that the best evidence indicated that attenuation is an $f^{2}$ dependence in this range of frequencies. Stoll and Bryan (1969) mention that for the attenuation of shear waves in highly permeable sands at frequencies ranging from $30 \mathrm{~Hz}$ to $340 \mathrm{~Hz}$, viscous losses due to relative movement of pore water and mineral may cause attenuation to be nearer $f^{2}$ at low frequencies. However, Hamilton (1980) mentions another study of the attenuation of shear waves in diluvial natural sands in situ at frequencies between $30 \mathrm{~Hz}$ and $80 \mathrm{~Hz}$ where it was possible to observe no velocity dispersion with $Q$ independent of frequency, and attenuation proportional to the first power of frequency.

In conclusion, several studies agree that there is an approximate first power dependence of attenuation on frequency in mud, from low to high frequencies. The attenuation estimation of sands in situ, in the low frequency range, is between the first and second power dependence of attenuation on frequency.

Hamilton (1980) mentions that the energy losses due to relative movement of pore water and sediment mineral frame, leading to an $f^{2}$ dependence on attenuation at low frequencies, may be of potential importance only in compressional and shear wave propagation in sands with rather uniform grains and high permeability. This is the more important case in Lake Maracaibo in the mud layer zone, which includes several layers of sands. McCann and McCann (1969) mention that for sediments of mean diameter less than $6 \phi$ (see equation (2.4)) the attenuation may be computed from the equation (2.20) assuming that the particles and water together behave as a fluid of density $1.35 \mathrm{~g} / \mathrm{cc}$ and viscosity of 0.015 poise. The non-surface active particles are suspended in the fluid and viscous dissipation of compressional waves occurs. For sediments of mean diameter greater than $6 \phi$, the viscous mechanism may dominate; for sediments at depth of burial of $2 \mathrm{~m}$, the solid friction mechanism is dominant. 


\subsubsection{Gassy Sediments}

The attenuation in gassy sediments occurs by the large acoustic impedance mismatch between the gassy sediments and the consolidated sediments. In addition, the gassy sediments and water together form a mechanical vibratory system that exhibits resonances at certain excitation frequencies. These resonance frequencies are in part determined by the bubble size.

Within the sea floor, a boundary between consolidated sediments and the gassy sediment horizon produces high acoustic reflectivity. Anderson and Bryant (1990) call this enhanced reflection "bright spots". Although other impedance contrasts can also cause a bright spot, a phase reversal of the reflected pressure wave at the bright spot narrows the interpretation and is a strong indication of probable gassy sediments. In some shallow water cases with very gassy horizons at shallow depths in the sea floor, most of the energy of a downward propagating acoustic wave will be reflected by the gassy layer. This reflected energy can then propagate upward to be re-reflected at the sea surface. Many repetitions of these low loss reflections can occur at the water/air and water-saturated sediment/gassy sediment interfaces. In the acoustic record many multiples of the initial string reflection from gassy horizons are observed. This is known as ringing.

Levin (1962) mentions that a similar behavior was noted in acoustic sounding records from Lake Maracaibo, and this was referred to as a "singing seismogram". In summary, the attenuation in gassy sediments is caused by frame friction absorption, and as for consolidated sediments, the absorption is due to loss mechanisms associated with bubble motion and scattering by the bubbles. Anderson and Hampton (1980b) show that the attenuation due to losses associated with bubble motion can be estimated using equations (2.21) and (2.22):

$$
\alpha=\frac{\pi c f}{c_{o}^{2}} \frac{K Y_{1}}{\gamma P_{o}+\frac{4}{3} G},
$$

where $c$ is the compressional wave velocity in gassy sediments, $K$ is the saturated sediment bulk modulus given by equation (2.8), $Y_{1}$ is defined in equation $(2.22), c_{o}$ is the compressional wave speed in the saturated sediment, $\gamma$ is the ratio of specific heats of gas, $G$ is the sediment rigidity modulus and $P_{o}$ is the ambient hydrostatic pressure. Anderson and Hampton (1980b) used equation (2.21) to obtain the attenuation curves 
shown in Figure 2.8:

$$
Y_{1}=\frac{n_{g}\left(d\left[\frac{f}{f_{o}}\right]^{2}\right)^{2}}{\left(1-\left[\frac{f}{f_{o}}\right]^{2}\right)^{2}+\left(d\left[\frac{f}{f_{o}}\right]^{2}\right)^{2}}
$$

where $n_{g}$ is defined as the gas porosity and is equal to the gas volume over the total sediment volume, $d$ is the damping of bubble motion in sediments, $f_{o}$ is the bubble resonance frequency, and $f$ is the frequency.

In Figure 2.8 both curves indicate an attenuation maximum near the bubble resonance frequency. Calculated attenuations are much higher in mud. These results indicate that attenuation can be very high in gassy sediments for frequency of resonance. If a distribution of bubble sizes is present, the attenuation will be large over a greater range of frequencies. A small quantity of gas can significantly increase the attenuation and the decrease the acoustic velocities. The gassy sediment attenuation predicted by equation (2.8) can explain several observations taken several years ago.

\subsection{Attenuation Estimation}

Lake Maracaibo presents a very complex system because the lake has the following: gassy sediments, viscous losses, solid friction losses, low resolution, hard reverberation or ringing, linear noise, guide waves and mud waves, etc. In addition, scattering and absorption are present due to the mud layer. Table 2.2 shows attenuation factors or energy damping of compressional and shear waves that are found in Lake Maracaibo in unconsolidated, consolidated and gassy sediments.

Figure 2.9 presents three different shot gathers obtained in Lake Maracaibo, and shows a change in the frequency from high to low when the seismic section is located in the mud area. The loss of frequency is evident in the mud zone. To illustrate this, the first shot gather is located in an area without the mud layer problems and the reflections between $3 \mathrm{~s}$ and $4 \mathrm{~s}$ are clear. However, the other two lines are located in different areas, with mud layer problems. In this case the reflections between $3 \mathrm{~s}$ and $4 \mathrm{~s}$ are not evident due to the signal/noise ratio decreased.

Because the attenuation as given by equation (2.11) is based on energy loss per oscillation for a given travel time, the high frequencies undergo more oscillations 


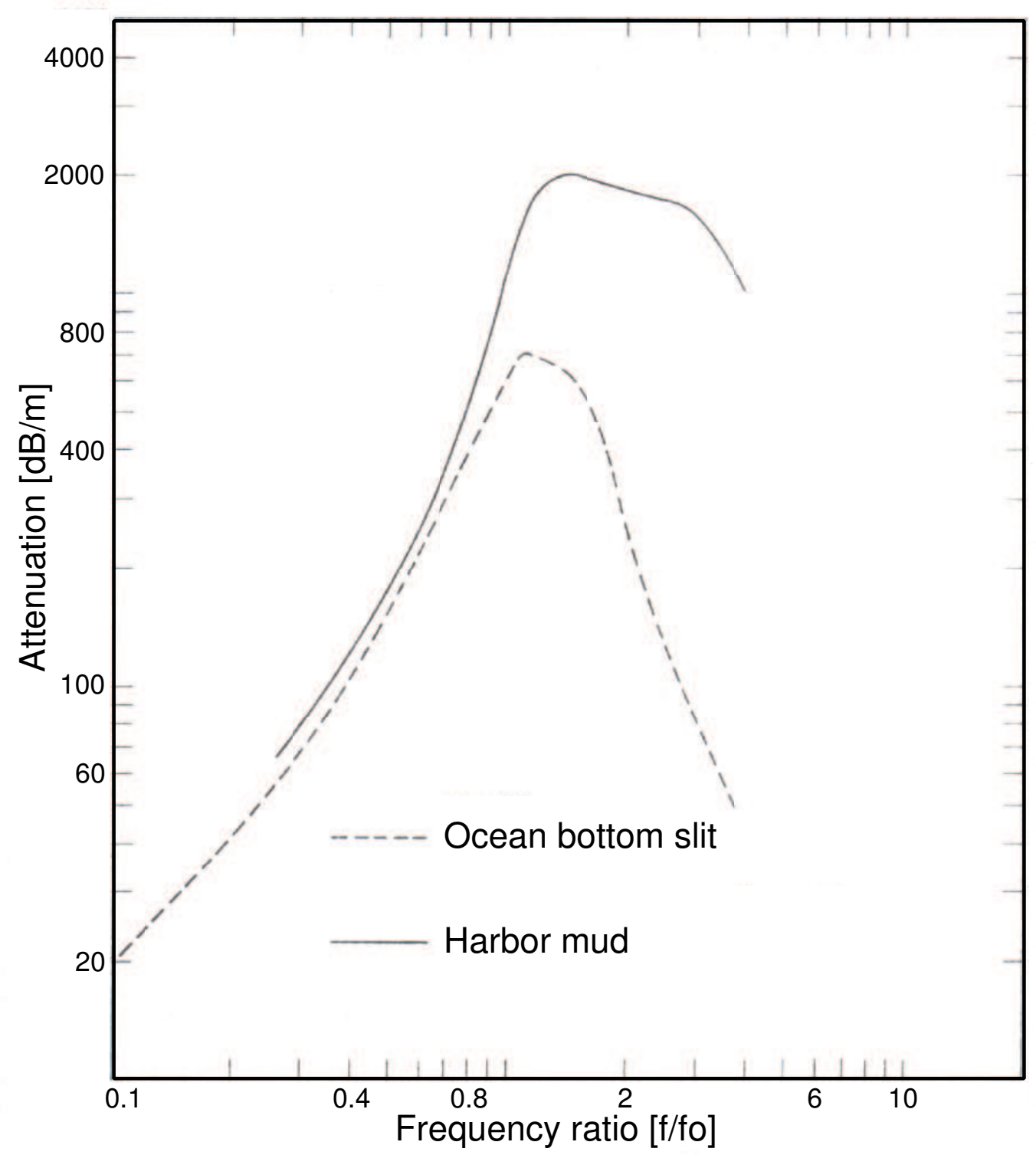

Fig. 2.8. Attenuation in gassy sediments versus frequency ratio. This frequency ratio is the frequency over the bubble resonance frequency. In this case two different sediments were used (Anderson and Hampton, 1980b). 




Fig. 2.9. Three different shot gathers from Lake Maracaibo. These seismic sections are from different seismic lines located in three different areas. The first location is an area without mud layer problems and the other two are in a zone with mud layer problems. Amplitude gain control was applied. 
Table 2.2. Principal attenuation factors in unconsolidated sediments, consolidated sediments and gassy sediments observed in Lake Maracaibo due to the mud layer zone.

\begin{tabular}{|c||c||c||c|}
\hline $\begin{array}{c}\text { Attenuation } \\
\text { Mechanism }\end{array}$ & $\begin{array}{c}\text { Unconsolidated } \\
\text { Sediments }\end{array}$ & $\begin{array}{c}\text { Consolidated } \\
\text { Sediments }\end{array}$ & $\begin{array}{c}\text { Gassy } \\
\text { Sediments }\end{array}$ \\
\hline \hline Geometrical Spreading & $\sqrt{ }$ & $\sqrt{ }$ & $\sqrt{ }$ \\
\hline Scattering & $\sqrt{ }$ & $\sqrt{ }$ & $\sqrt{ }$ \\
\hline Absorption & $\sqrt{ }$ & $\sqrt{ }$ & $\sqrt{ }$ \\
\hline Viscous losses & $\sqrt{ }$ & $\sqrt{ }$ & \\
\hline Solid friction losses & $\sqrt{ }$ & $\sqrt{ }$ & \\
\hline
\end{tabular}

than do the low frequencies. The higher frequencies are preferentially attenuated, and wavelet shape changes with time.

There are a variety of techniques by which $Q$ can be measured from field seismic data, all based on this phenomenon. For a source wavelet that is symmetric in time, the peak frequency is also the central frequency, and the instantaneous frequency from complex analysis may be used. Other methods include rise-time analysis, spectral ratios, inverse $\mathrm{Q}$ filtering and wavelet modeling in the time or frequency domain (Bourbie et al., 1987).

A significant obstacle in modeling $Q$ using any of these methods is the interference between layers that are separated by less than a pulse length in arrival time. This interference produces a composite signal with a frequency content lower than that of the individual contributing signals. The magnitude of this effect depends on the relative reflection strengths and layer thicknesses, and is generally unpredictable.

Edrington and Callowat (1984) showed a procedure to calculate the compressional wave attenuation. The method is based on the difference between the amplitude spectra of the signals at two hydrophones located at different positions from the source. Following Edrington and Callowat (1984), the compressional wave attenuation can be written as follows:

$$
k\left(d_{b}-d_{a}\right) f+C_{a b}=20 \log \left(\frac{S_{a}(f)}{S_{b}(f)}\right),
$$

where the coefficient $k$ is a constant that has units of $\mathrm{dB} / \mathrm{kHz}-\mathrm{m}$, and this has been 
evaluated for saturated sediment attenuation data by Hamilton (1972). $d_{a}$ and $d_{b}$ are the respective propagation path lengths; $S_{a}$ and $S_{b}$ are the spectra of the signal for the hydrophones; and $C_{a b}$ is a constant that accounts for spreading losses and calibration errors. The objective is to estimate $k$ under the assumption that attenuation is indeed proportional to frequency. Edrington and Callowat (1984) used this procedure to get the compressional wave attenuation in the Mississippi Delta. The measurements were taken in soft to very soft dark gray clay sediments with a water content of $70 \%$ to $90 \%$ and cohesive shear strength generally less than 300 psf. Gas was reported at depths below $6 \mathrm{~m}$, the water depth was $22 \mathrm{~m}$, and the temperature at the mud line was $24.8^{0}$ C. Edrington and Callowat (1984) estimated a value for $k$ about $1.4 \mathrm{~dB} / \mathrm{kHz}-\mathrm{m}$ that was an order of magnitude greater than typical values of $k$ for non-gassy sediments. Consequently gassy sediments may quickly approach acoustic opacity as thickness or frequency increases.

\subsection{Gas Content Estimation}

Many sediments contain organic matter, in addition to the inorganic particles. The particles of organic matter usually have a specific gravity only slightly greater than water. However, some organic matter is in the form of fatty or amino acids adhering to clay particles. Organic material will tend to be deposited in areas of low water movement energy. These areas are also where the smaller inorganic material is deposited.

Anderson and Hampton (1980a) mentioned that the organic material in sediments may be decomposed by bacteria. If oxygen is available, aerobic decomposition will prevail, producing water and carbon dioxide. However, if oxygen has been depleted, anaerobic decomposition occurs. Although aerobic decomposition is most efficient, a centimeter or less below the sediment/water interface, oxygen is almost always absent and thus anaerobic decomposition occurs.

In synopsis, the output of the bottom gassy sediment chemical system consists of gaseous small organic molecules that dissolve in the interstitial aqueous medium. In a situation in which gases are continually produced by bacteria, the gaseous product concentration will continue to increase in the interstitial water until saturation is reached. Once the initial bubbles are formed, other gases in solution will diffuse across the bubble boundaries until diffusion equilibrium is reached. 
Anderson and Hampton (1980a) mentioned that the acoustic gassy sediments can be described as a function of the three different parameters: size distribution, internal pressure and the ratio of specific heats of the gases. The average of the ratio of specific heats of the gassy products is 1.30 . The internal pressure of the bubbles will equal the interstitial water pressure plus bubble surface tension, and is thus a function of the overall water depth. The minimum bubble size may be estimated by considering the minimum size of bubbles in water. The internal pressure of a bubble of equilibrium size is equal to the external hydrostatic pressure plus the pressure resulting from surface tension at the bubble/water boundary.

Anderson and Hampton (1980a) showed that for a spherical bubble of radius $r$ and surface tension $T_{s}$, the surface tension pressure is given by $2 T_{s} / r$. In this context, the pressure increases as the bubbles' radius decreases, thus the pressure tends to drive gas in the bubble into solution as the bubble size decreases.

Edrington and Callowat (1984) described a study where the speed and attenuation of compressional waves in gassy marine sediments in the Mississippi Delta area were measured by means of small explosive charges buried $30 \mathrm{~m}$ below the mud line. In this study, the Sandia National Laboratories tested a gun-launched instrumented sea bed source system that provided a capability to emplace payloads at depths of at least $50 \mathrm{~m}$ in soft marine sediments. The data set information from the source was telemetered to the surface by means of acoustic pulses generated by small explosive charges.

These instruments provided a means of measuring the propagation properties of the medium between the source and receiver. The characteristics of the received signal can then be used to make inferences about the speed and attenuation of sound in marine sediments. The means size of particles at a depth of $30 \mathrm{~m}$ was between 12 $\mu \mathrm{m}$ and $23 \mu \mathrm{m}$. The average speed of propagation in the sediments above the source was determined by measuring the time intervals between the detonation of the first signal charge and the first arrivals of sound at the hydrophones.

Edrington and Callowat (1984) showed that the compressional wave velocity estimate can be used to make an inference about gas content through Gassmann's equation. There are several conditions for using this method to get the gas content in the study area.

The first requirement is that the shear modulus of the sediments has to be much smaller than the bulk modulus. The second is that the presence of gas has a negligible 
effect on bulk density. In this way, the ratio of the compressional wave velocity in the gassy sediments to that in a non-gassy but otherwise similar sediment is approximately the square root of the ratio of the respective bulk modulus.

When the stress and strain relations are written in terms of the Lamé's constants, it is possible to get the velocity wave equation for compressional and shear waves. When the shear modulus $\mu$ is equal to zero, only a single wave propagates, and only the compressional wave is observed. The ratio of the propagation velocity between gas and fluid can be written as follows:

$$
\begin{aligned}
\frac{\alpha}{\alpha_{o}} & =\sqrt{\frac{K \rho_{o}}{K_{o} \rho}}, \\
\rho_{o} & \approx \rho, \\
\frac{\alpha}{\alpha_{o}} & \approx \sqrt{\frac{K}{K_{o}}}
\end{aligned}
$$

where $\alpha$ and $\alpha_{o}$ are the compressional wave velocities in gassy sediments and fluid respectively. $K_{o}$ is the fluid phase bulk modulus and $K$ is the bulk modulus of the gas phase. For partially saturated rocks at frequencies less than $100 \mathrm{~Hz}$, it is possible to obtain an effective modulus for the pore fluid that is an average of the moduli of the liquid and gaseous phases. Gassmann (1951) obtained an expression relating the total sediment bulk modulus, $K_{s}$, to the mineral bulk modulus, $K_{m}$, the interstitial water bulk modulus, $K_{w}$, and the sediments frame bulk modulus, $K_{f}$. In this expression if the frame bulk modulus is set equal to zero, it is possible to get the compressibility of the gassy sediment equation, which can be written as follows:

$$
K^{-1}=n K_{g}^{-1}+(1-n) K_{o}^{-1},
$$

where $n$ is the gas content and $K_{g}$ is the bulk modulus of the gas phase. For the typical pressure variations for the wave propagation, the bulk modulus of the gas can be written as:

$$
K_{g}=\gamma P_{o},
$$

where $P_{o}$ and $\gamma$ are the pressure of the gas and the ratio of specific heat of the gas, respectively. In the equation (2.25), it was assumed that the expansion has no net gain or loss of heat in the system. The Gassmann's expression reduces to the suspension bulk modulus equation when the frame bulk modulus is set equal to zero and is used 
to make an assumption about gas content and can be written as follows:

$$
\frac{\alpha}{\alpha_{o}} \approx\left(1+n\left(\frac{K_{o}}{\gamma P_{o}}-1\right)\right)^{-1 / 2} .
$$

Table 2.3 shows the effective gas content estimated using the gassy sediment velocities observed in the mud layer area in Lake Maracaibo. The conclusion is that the sediments are not homogeneous and there were some layers with considerably more than $1.89 \%$ gas, and others, especially near to the lake surface, with considerably less than $0.18 \%$ gas.

Table 2.3. Typical values for the bulk modulus of the fluid, hydrostatic pressure, fluid velocity and gassy sediment velocity. The effective gas content, when the gassy sediment velocities are $300 \mathrm{~m} / \mathrm{s}$ and $700 \mathrm{~m} / \mathrm{s}$, are $1.89 \%$ and $0.18 \%$ respectively (Levin, 1962).

\begin{tabular}{|c||c||c||c||c||c||c|}
\hline$K_{o}$ & $P_{o}$ & $\gamma$ & $C_{o}$ & $C$ & $N_{300}$ & $N_{700}$ \\
\hline \hline $3 x 10^{9} \mathrm{~N} / \mathrm{m}$ & $1 x 10^{6} \mathrm{~N} / \mathrm{m}$ & 1.3 & $1500 \mathrm{~m} / \mathrm{s}$ & $300 \mathrm{~m} / \mathrm{s}$ & $1.89 \%$ & \\
\hline $3 x 10^{9} \mathrm{~N} / \mathrm{m}$ & $1 x 10^{6} \mathrm{~N} / \mathrm{m}$ & 1.3 & $1500 \mathrm{~m} / \mathrm{s}$ & $700 \mathrm{~m} / \mathrm{s}$ & & $0.18 \%$ \\
\hline
\end{tabular}

In Lake Maracaibo, the gassy sediment reduces the compressional wave velocity from $1500 \mathrm{~m} / \mathrm{s}$ to between $300 \mathrm{~m} / \mathrm{s}$ and $700 \mathrm{~m} / \mathrm{s}$. By solving equation (2.27) with the typical values of the bulk modulus and velocity for the non-gassy sediments and estimated hydrostatic pressure, it is possible make an estimate of the gas content in Lake Maracaibo. 


\section{CHAPTER III}

\section{LAKE MARACAIBO FEATURES}

The Maracaibo Basin is the second most petroliferous basin in the world. Approximately 17,000 wells have produced 37 billions barrels since 1914. Remaining recoverable reserves are nearly 20 billion barrels, and the estimated original total of oil and gas in place is as high as 320 billions barrels and 90 TCF (trillion cube feet), respectively. The area evolved from a Cretaceous to Eocene (transtensional) back-arc basin, to a (transpressive) forehand basin during post-Eocene time. Hydrocarbon, mainly from the Upper Cretaceous source rock, is now found in fractured Cretaceous carbonates (6\%), Eocene clastics (50\%), and Miocene clastics (44\%). Most oil accumulation in the west and northwest are related to faulted anticlines, while in the east, structures are generally homoclinal and accumulation is largely due to truncation and lenticularity of the Miocene reservoir beds, and a tar seal at the Miocene outcrop. Often oil is trapped in the faulted and truncated Eocene. The predominant mud sediments are very loosely deposited, and are therefore of rather low density. The average mud density is $1.28 \mathrm{~g} / \mathrm{cc}$ and the porosity is between $40 \%$ and $80 \%$.

\subsection{Geographical Location and Geology}

Figure 3.1 shows the location of Lake Maracaibo. The largest lake in South America is a huge inlet of the Caribbean Sea in the northwestern corner of Venezuela. Covering an area of approximately $13,350 \mathrm{~km}^{2}$, it is about $210 \mathrm{~km}$ long by $120 \mathrm{~km}$

at its widest. Situated in a basin between the Sierra de Perija range in the west and the Cordillera de Mérida range in the east, the oval-shaped lake is nearly landlocked. The lake is quite shallow, with a maximum depth of less than $50 \mathrm{~m}$. It is connected to the sea by the Straits of Maracaibo, a narrow canal about $60 \mathrm{~km}$ long and $15 \mathrm{~m}$ deep. Recently deposited marine sediments in the bay have reduced the depth almost everywhere to less than $45 \mathrm{~m}$. Figure 3.2 shows the location of the mud layer zones and the three seismic lines.

The Maracaibo Basin is located within a triangular tectonic block bounded by three major strike slip faults. These faults are the result of northeast compression 


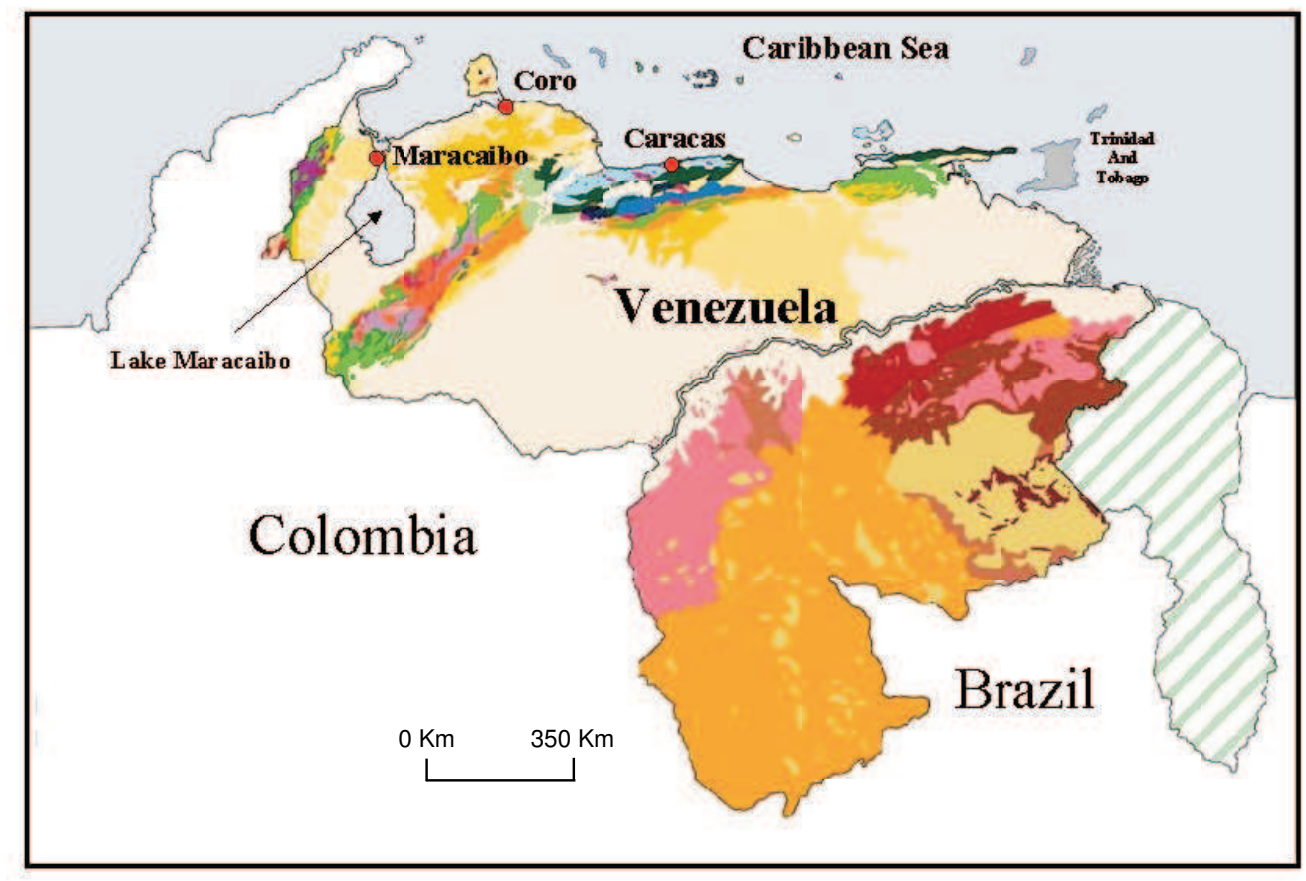

Fig. 3.1. Geological map from Venezuela. Lake Maracaibo is in the northwestern corner of Venezuela.

exerted by the Nazca Plate against northwestern South America, and dextral movement between the Caribbean and South American Plates. Within the Maracaibo Basin are a series of sinistral wrench faults along which transpression has occurred. The main stream is the Catatumbo River, the delta of which is a prominent feature of the southwestern corner of the lake. Overall the lake bottom is nearly flat, although it has irregularities, and several studies show that these bottom features consist of fairly large elongated depressions, smaller nearly circular holes and small mounds (Sarmiento and Kirby, 1962).

The geological history of the recent sediments begins with the Wisconsin glacial period, where water covered a very small portion of the present lake. Probably the ancient lake area consisted mainly of swamps, a wetland often partially or intermittently covered by water, where peats were accumulating. Lake Maracaibo was drained through the Maracaibo Straits and Tablazo Bay, and the form of the straits suggests a river valley carved under semiarid conditions. Sarmiento and Kirby (1962) mention that the deepest peat found indicates that the minimum lake level was at least $45 \mathrm{~m}$ below the present sea level. The sea level at the close of the glacial period, as 


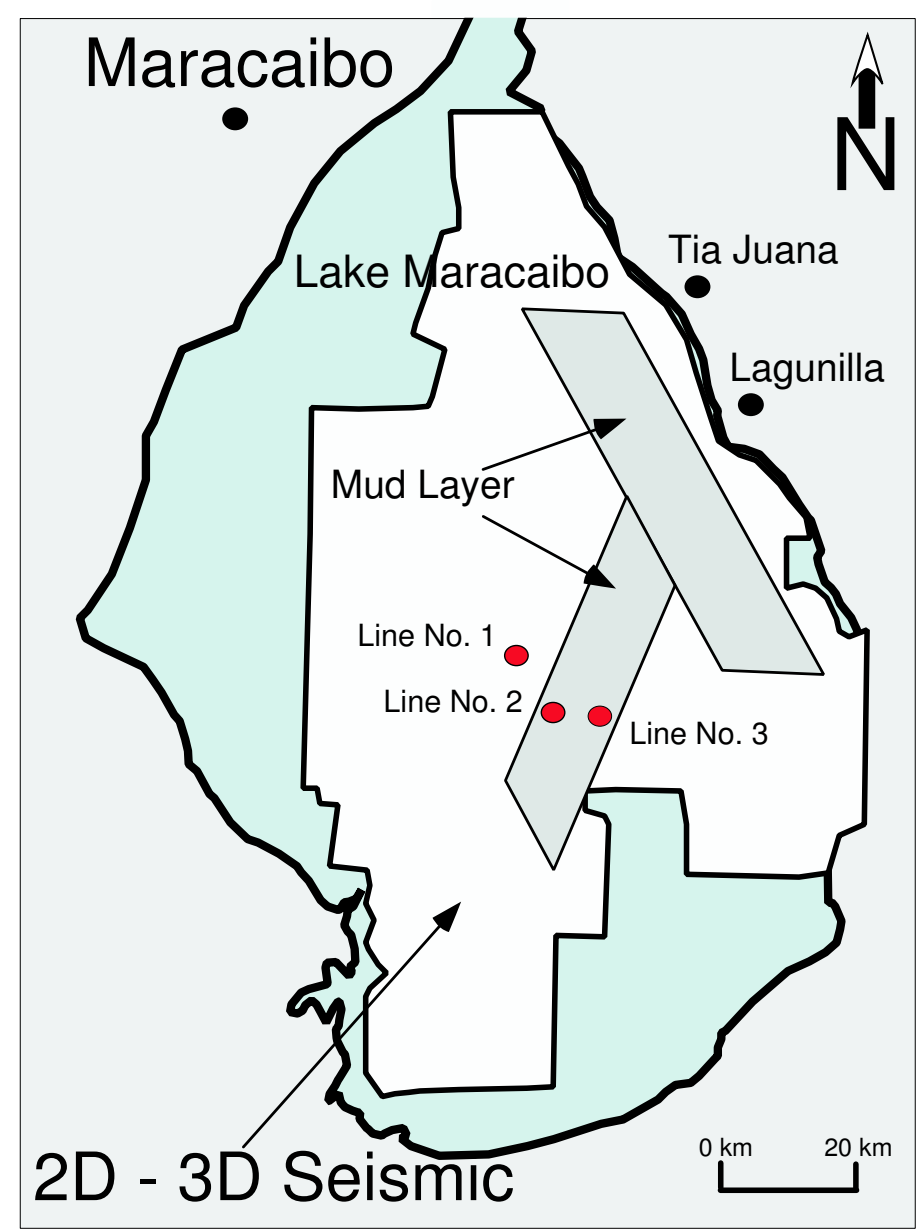

Fig. 3.2. Lake Maracaibo map. It is possible to observe the location of the three seismic lines used in mud layer study. The relative location of the mud layer zones are shown.

interpreted from Pleistocene geology, was correspondingly lower. As shown by the age of succeedingly higher peats, the lake level slowly rose. Thus, several studies of different peat samples indicate that the lake level rose at a rate of about $0.5 \mathrm{~cm}$ per year, between 9,270 years ago and 7,370 years ago.

During the early stages of the rising sea level, Tablazo Bay was flooded. This transgression started the deposition of the marine sediments that today constitute the main barrier defending the lake from the sea water. During the early postglacial epoch, the lake was fresh but, as indicated by the diatomite, eventually the barrier of marine sediments of Tablazo Bay did not keep pace with the rising sea level, thus permitting appreciable quantities of salt water to enter the lake. The absence of pelagic foraminifera and other marine remains within the diatomite deposits suggests 
brackish rather than marine conditions (Sarmiento and Kirby, 1962). After the lake attained maximum salinity, it became progressively less saline until it reached its present state.

The recent sediments of Lake Maracaibo attain a thickness of as much as $60 \mathrm{~m}$, depending on location, and consist predominantly of very fine-grained, loosely deposited, dark greenish gray muds. Median grain diameters are in the order of the 1 micron (Sarmiento and Kirby, 1962). Mud densities vary between $1.20 \mathrm{~g} / \mathrm{cc}$ and $1.40 \mathrm{~g} / \mathrm{cc}$, and the porosities vary between $40 \%$ and $80 \%$. Interbedded with the mud layers is a widespread layer of diatomite. Peat is found in the lower part of these sediments, and fine sands, generally micaceous, are in some of the near shore shallow areas. The lake bottom is nearly flat over two-thirds of its surface. The flat bottom covers the central and eastern portions, and corresponds to water depths of about $45 \mathrm{~m}$. A narrow, relatively steep shoreward rising of the lake bottom characterizes the eastern and southern borders.

Paleontological evidence shows that between 7,000 and 10,000 years ago the lake changed from a fresh to a brackish body of water. Several studies show that gassy sediment, most of which is derived from organic material, occurs widely in the lake. This is to be expected in such an environment, where the decay of the abundant organic matter produces methane, carbon dioxide and nitrogen. This organic matter arrives at the lake floor from both local lake productivity and terrigenous sources. If oxygen is available, oxidation reactions descompose the organic material. The balance of oxidation rates, supply of organic material and sedimentation rates determines the final sedimentary organic matter content after oxygen has been depleted. Another factor in this balance is the extent of bioturbation, and thus reoxygenation of the sediment. At shallow depths of burial, but in the absence of oxygen, decomposition of organic material is primarily mediated by anaerobic bacteria. Figure 3.3 is a Lake Maracaibo near surface cross-section, a general view of the different layers in this zone. The zone described as mud, in Figure 3.3, represents the area where the largest concentration of gassy sediments is found. The mud layer changes in thickness laterally, and the concentration distribution and quantities of the gas are unknown.

High organic carbon content is found in several areas in Lake Maracaibo, with the content found in both the center and in the southern part of the lake. However, it is possible to find high organic carbon content in the deeper central area. Mud sediments from the shallower areas, the northwest portion of the lake, northeast 


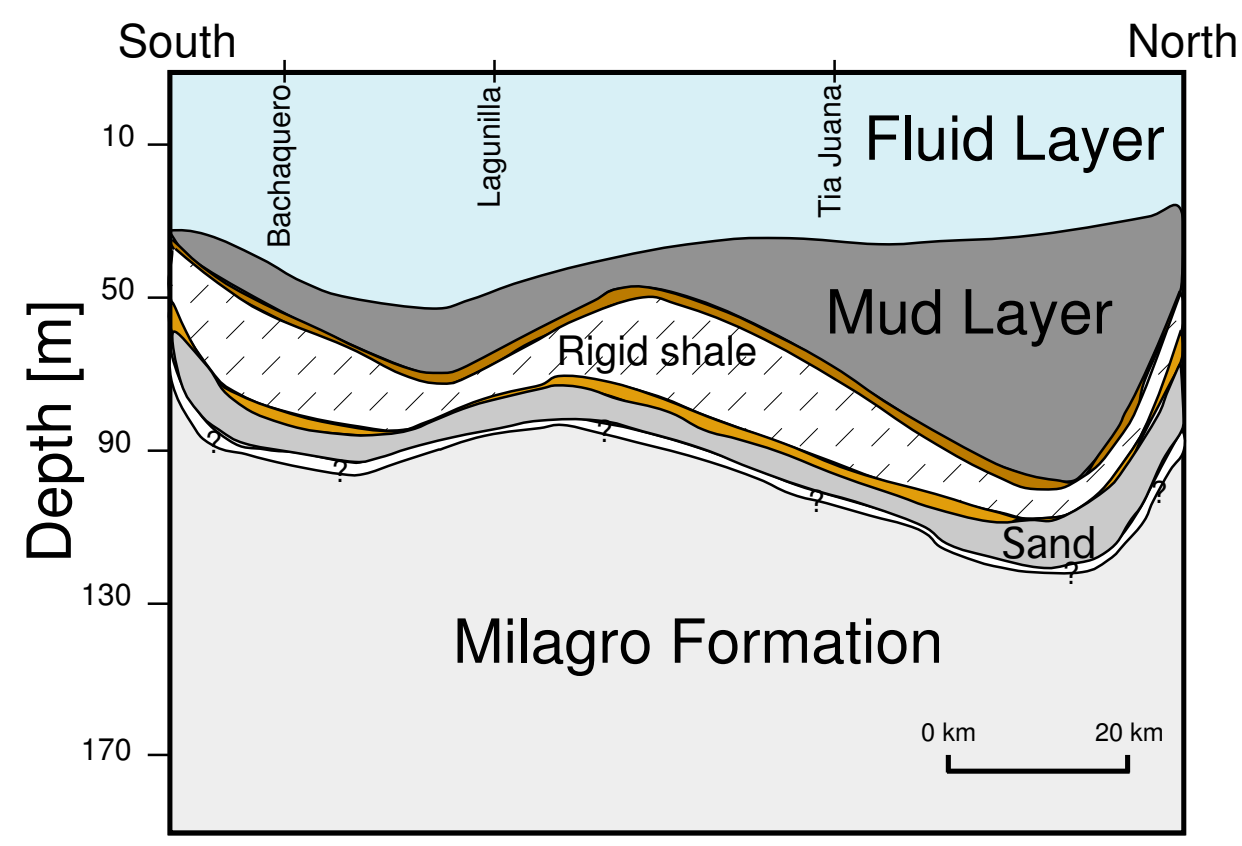

Fig. 3.3. Near surface cross section of Lake Maracaibo. This is a simplification of the different strata observed in the area. The gassy sediments are principally in the mud layer (Michelena et al., 1998).

portion of the lake and southeast part of the lake show about half the carbon content of the deeper muds. The Milagro clays contain the lowest amount of organic carbon. The hydrocarbon concentration is low in almost all the lake, between $0.1 \%$ and $0.4 \%$. These low values are characteristic of peats, coals and similar continental sediments (Sarmiento and Kirby, 1962).

Sarmiento and Kirby (1962) published Table 3.1, which represents the results of the gas analysis on an air free basis. This study showed that the gas content in Lake Maracaibo varies widely and that the content is enough to reduce the compressional wave velocity below the value for the consolidated sediment. Consequently this causes an increase in the attenuation of the compressional wave propagating through this area. This result is to be expected in such an environment, where decay of abundant organic matter produces methane, $\mathrm{CH}_{4}$. Carbon dioxide, $\mathrm{CO}_{2}$, and nitrogen, $\mathrm{N}_{2}$, are probably derived from bacterial action.

The measurement of ultrasonic velocities of the lake bottom sediments and estimation of velocities from pile records show a range of velocities between $300 \mathrm{~m} / \mathrm{s}$ and $1440 \mathrm{~m} / \mathrm{s}$. Vertical and horizontal variation in the mud layer is observed in the center lake area, producing an attenuation of the high frequency in the compressional wave. 
Table 3.1. Gas content in the recent mud sediments. The samples were taken in the northeast portion of the lake [Core No. 1] and in the center of the lake [Core No. 2] (Sarmiento and Kirby, 1962).

\begin{tabular}{|l||r||r|}
\hline Sample gas & Core No. 1 & Core No. 2 \\
\hline \hline Depth of sample & $21 \mathrm{~m}$ & $31 \mathrm{~m}$ \\
\hline Total volume & $800 \mathrm{ml}$ & $800 \mathrm{ml}$ \\
\hline Total average of $\mathrm{CH}_{4}$ & $58 \mathrm{ml}$ & $63 \mathrm{ml}$ \\
\hline Total average of $\mathrm{CO}_{2}$ & $77 \mathrm{ml}$ & $77 \mathrm{ml}$ \\
\hline Total average of $\mathrm{N}_{2}$ & $9 \mathrm{ml}$ & $13 \mathrm{ml}$ \\
\hline \hline Total gas by volume & $144 \mathrm{ml}$ & $154 \mathrm{ml}$ \\
\hline
\end{tabular}

Figure 3.4 shows a shear wave velocity plot estimated from the pile records. This figure is an example of the velocity change in depth over hard stratum just below the mud layer for three different locations in Lake Maracaibo.

The variation in the shear velocity in these strata can be observed. These velocities were estimated using correlations between shear wave velocity measurement in core and pile records obtained in layers from the eastern section of Lake Maracaibo next to coast (Michelena et al., 1998).

Sarmiento and Kirby (1962) studied the sediments of Lake Maracaibo. This study included forty cores ranging in length from 2 to $5.5 \mathrm{~m}$ in six different areas. This distribution of the areas gave poor coverage of the eastern half of the lake. Figure 3.5 shows a generalized stratigraphic column. The substratum, unit $A$, is believed to belong to the upper part of the Milagro Formation and is probably of Pleistocene Age. It is a mottled silty clay that generally shows brownish or yellowish iron oxide coloration. The average density of the clay is $2.0 \mathrm{~g} / \mathrm{cc}$ and its water content by volume is about $40 \%$.

Unit $B$ is a lower sequence of the recent sediments that is of varying lithology. It is made up of soft, medium dark gray clay, micaceous sand and silt, with occasional peat layers. There is no lithologic difference between units $C_{1}$ and $C_{2}$. They have been split because of the existence of a diatomite, unit $D$, interbedded between the recent muds. The muds are dark greenish-gray, dark olive gray, and grayish olive green, and were deposited very loosely. They are extremely fine-grained, being pre- 


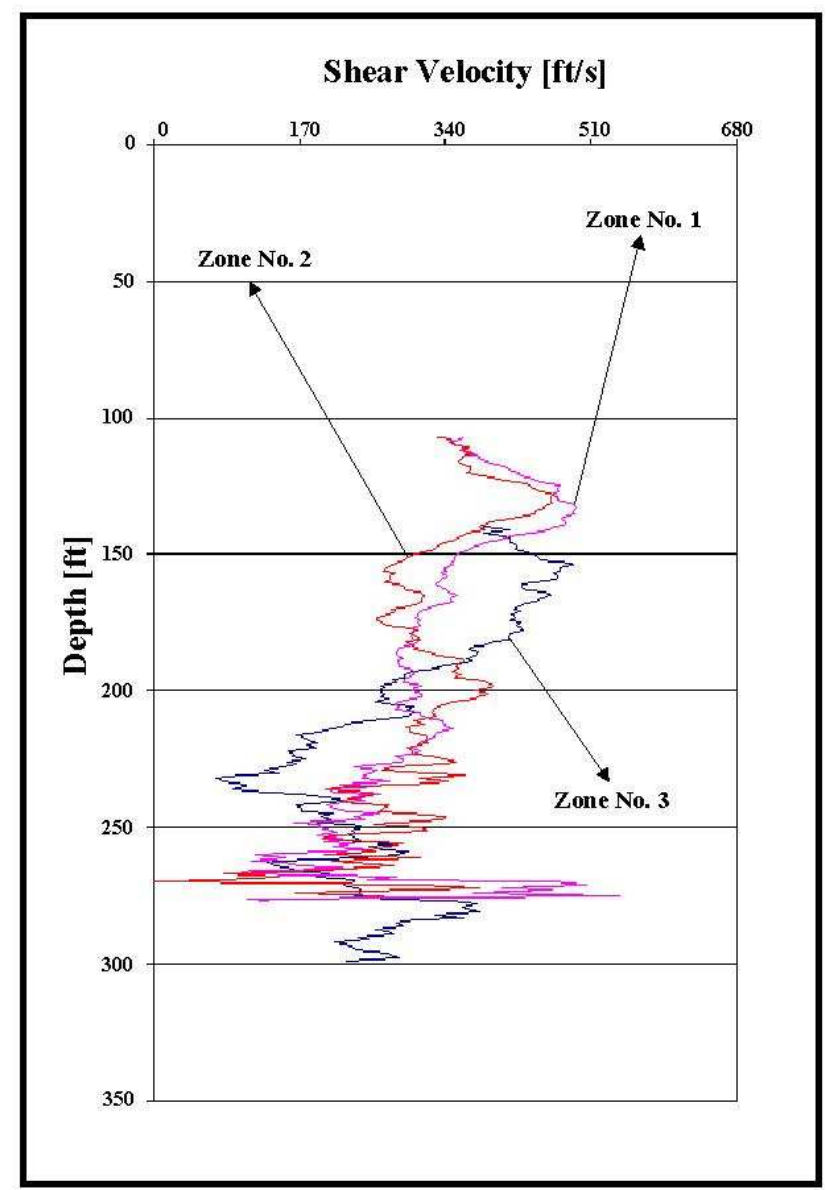

Fig. 3.4. Estimation of the shear velocity in depth from the pile records. These velocities are averages for three different areas in Lake Maracaibo, where the mud layer is present.

dominantly clay muds and silty clay muds with median diameters between $5 \times 10^{-4}$ and $3 \times 10^{-3} \mathrm{~mm}$. These units have densities varying between $1.20 \mathrm{~g} / \mathrm{cc}$ and $1.53 \mathrm{~g} / \mathrm{cc}$. Unit $D$, interbedded with the recent muds, is an olive gray diatomaceous earth sequence, which is typically very thinly layered. The contact of the diatomite with the overlying and underlying muds are transitional, grading into banded diatomaceous muds.

The contact between the recent sediment, mud layer and the substratum, Unit $A$, is unconformable, and this unconformity is one of the most interesting features of the Quaternary geology of Lake Maracaibo. The sediments in the lake are extremely fine-grained; the average median diameter of the recent muds is $2.81 \phi$ and vertical size variations were observed. 


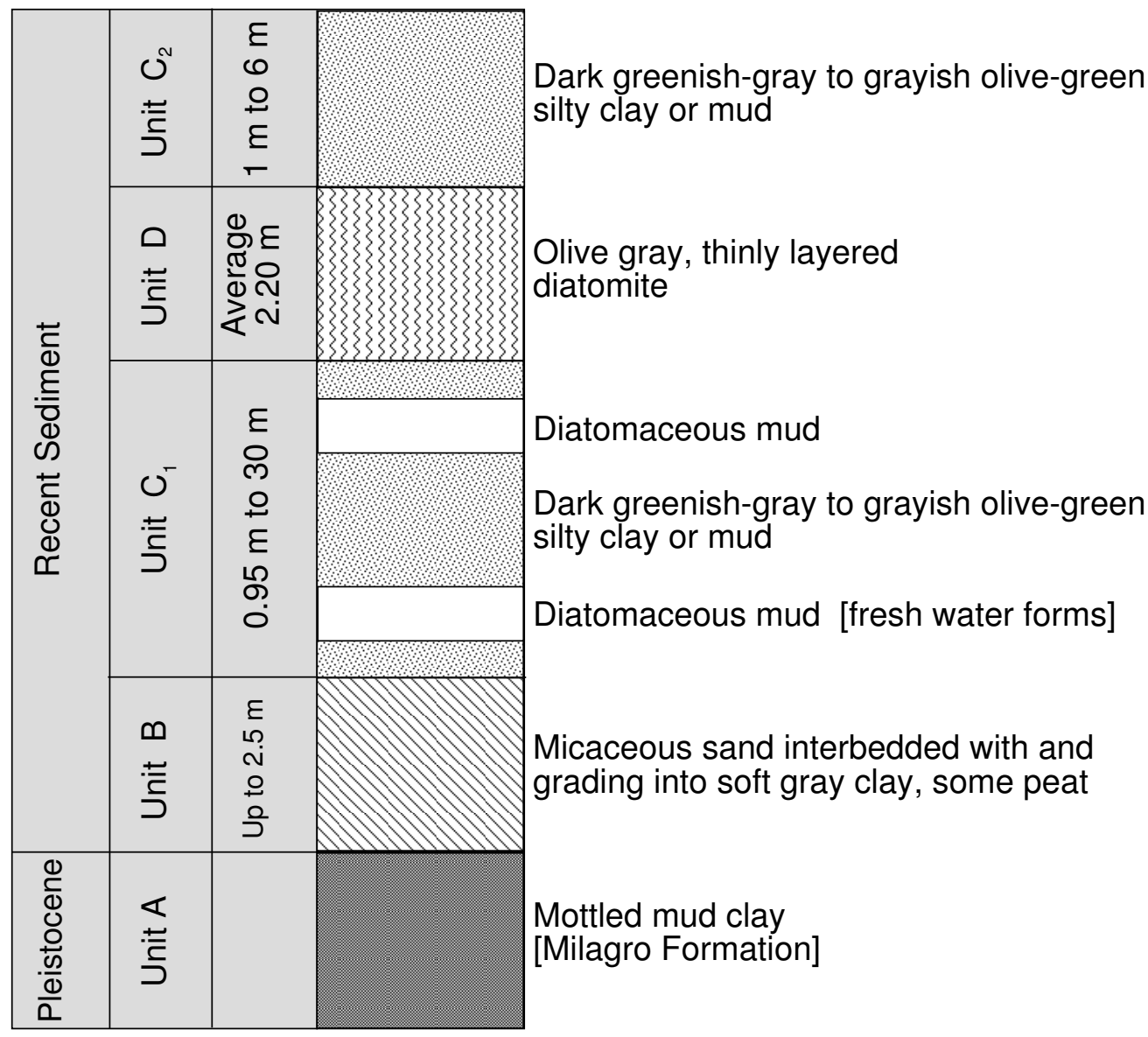

Fig. 3.5. Generalized stratigraphic column of recent sediments of Lake Maracaibo (Sarmiento and Kirby, 1962). The sediment studied included a clay substratum [Unit A], probably Pleistocene, and the recent sediments divided into four stratigraphic units.

The unconsolidated sediments, mud, slits and sands, show a close relationship between porosity and grain size, similar to what is shown in Figure 2.3. On the other hand, the effect of grain size on porosity is very minor in the more consolidated clays of the Milagro Formation. The relationship between bulk densities and porosities of consolidated sediments is almost linearly inverse, where the density decreases when the porosity increases.

Is well known that porosity is the most important factor affecting velocity in unconsolidated sediments (Table 2.1). The relationship between compressional wave velocity and porosity, in Lake Maracaibo unconsolidated sediments, shows a clear decrease of velocity with increasing porosity, similar to what is observed in Figure 2.1. The mud sediments, without gas bubbles, have compressional wave velocities 
below that of water, and porosity of more than 70\%. Additionally, the acoustic impedance analysis of Lake Maracaibo sediments shows that since both density and velocity decrease as porosity increases, the impedance also decreases. Sarmiento and Kirby (1962) mention that, in Lake Maracaibo, the relationship between impedance and porosity shows less scatter than the relationship between velocity and porosity, where differences were found among unconsolidated sediments, sands and clays.

In summary, the bottom sediments in Lake Maracaibo are loosely deposited and consist mainly of very fine-grained muds. The thickness of this mud layer changes as a function of the position, where the maximum thickness is approximately equal to $40 \mathrm{~m}$, with an average of $25 \mathrm{~m}$. These recent muds rest upon an uneven surface formed by a tough clay. There is a sharp density contrast between the mottled clay, with a density of about $2.0 \mathrm{~g} / \mathrm{cc}$, and the unconsolidated muds lying above, which have an average density of $1.28 \mathrm{~g} / \mathrm{cc}$. The mottled clay is often overlain by a micaceous sand. Peat layers were also locally developed above the clay layer. Above the sands, characteristic muds occur. They are of dark greenish-gray to grayish olive green color and are so loosely deposited that their porosity averages approximately equal $80 \%$.

These muds are extremely fine-grained where the median diameter of the particles that form them is close to $3 \phi$. The mud layer contains appreciable quantities of methane, carbon dioxide and nitrogen. The measured compressional wave speed was between $300 \mathrm{~m} / \mathrm{s}$ and $700 \mathrm{~m} / \mathrm{s}$. This change in the velocity in the mud is due to the gassy sediment localized in the mud layer zone. This low mud compressional wave speed results in a large reflection coefficient at the mud/water interface, with a phase reversal. In general, the sands are denser, $1.8 \mathrm{~g} / \mathrm{cc}$, and less porous, $52 \%$. The porosities and densities of the lake sediments are related by a nearly linear function. In the unconsolidated sediments, porosity generally increases as grain size decreases. There is less scattering in the correlation of acoustic impedance and porosity than in that of velocity and porosity.

Figure 3.6 shows a layer configuration used to represent the first 110 meters in the Lake Maracaibo zone. These configuration layers attempt to simulate the viscous losses, solid friction losses and gassy sediments zones with extreme velocity change and hard sediments layer in the mud layer. To establish the parameters in the mud layer zone, several synthetic seismograms with different attenuation factors will be utilized. 


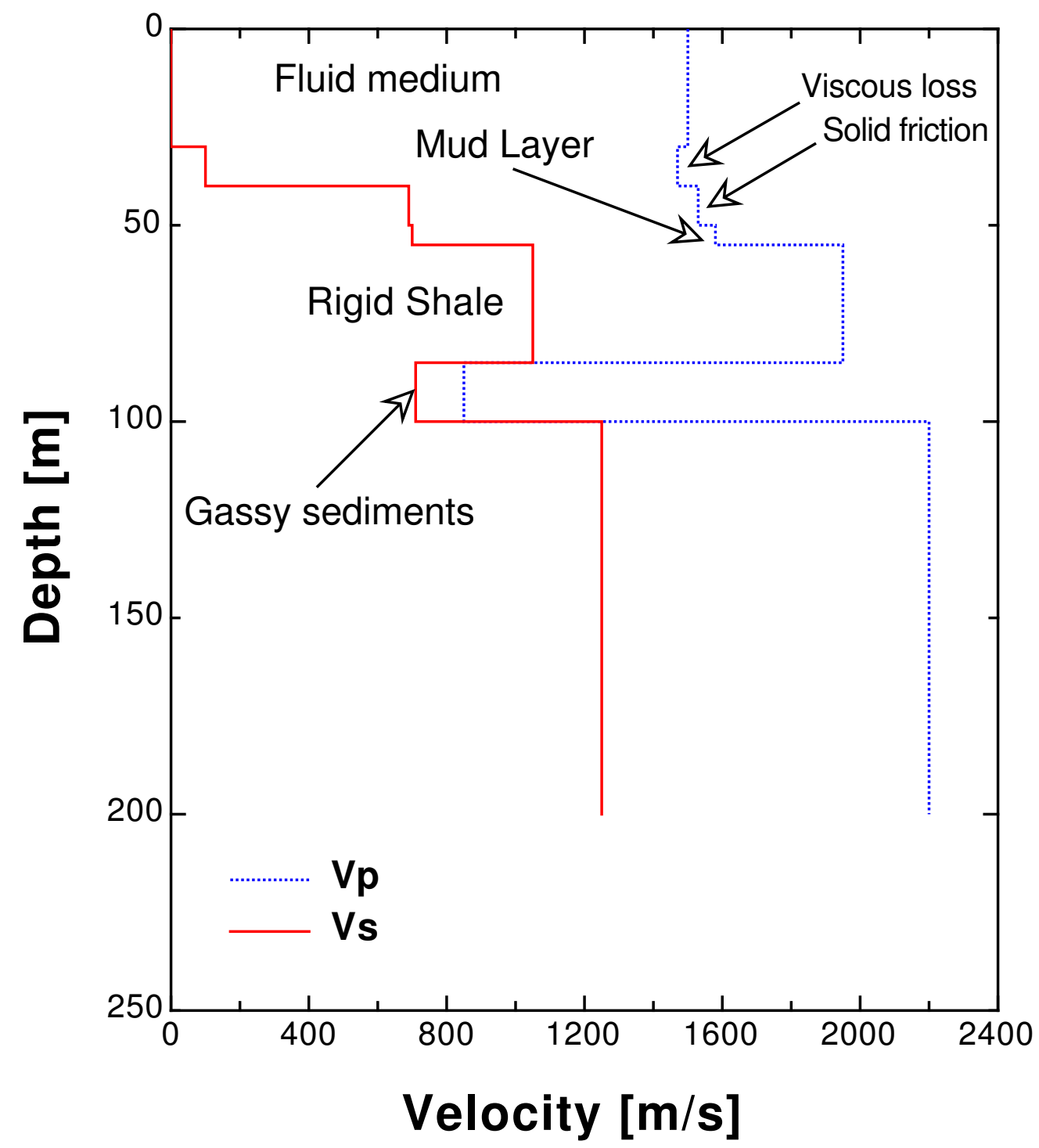

Fig. 3.6. Velocity profile used to build the synthetic seismogram in Lake Maracaibo for the mud layer zone. Three different kinds of attenuation mechanisms are considered: viscous losses, solid friction losses and gassy sediment effect. 


\subsection{Seismic Features}

The quantity of gas in sediment depends on the production rate, which in turn is a function of several variables. Bacteria that perform decomposition are present in virtually all natural aqueous media. Although mineralization of organic matter reduces the amount available for decomposition, very small quantities of organic matter produce large quantities of gas. In Lake Maracaibo, the highest concentration of gas is due to carbon dioxide, but it is possible to find methane, nitrogen and other gasses. All of these gasses originate from oxidation reactions that proceed to decompose the organic material, biogenic activity and anaerobic bacteria. The gas from biogenic activity and anaerobic bacterial processes is primarily within the top few meters of sediments. Lake Maracaibo is an area with rapid sedimentation, and gas is buried in depths below where it was generated.

In Lake Maracaibo the gas can occur in the lake bed in two ways: in solution in the pore water and undissolved in the form of gas filled voids. In these two situations, the gas will affect the acoustic properties of the lake bed and can produce change in the compressional wave speed, high attenuation of the compressional waves propagating through this medium, increases in the acoustic reflection, etc. Additionally, all these acoustic properties exhibit frequency dependent variation. However, other seismic phenomena can be observed, such as decreased resolution as depth increases, linear noise, hard reverberation or ringing, guide waves or channel waves, etc.

In Lake Maracaibo the amount of gas in the mud layer is sufficient to give, at low frequencies, compressional wave velocities much less than those of either water or water-saturated sediments. Levin (1962) showed that samples taken $4 \mathrm{~m}$ below the bottom contained enough gassy sediments to produce sound velocities between $260 \mathrm{~m} / \mathrm{s}$ and $670 \mathrm{~m} / \mathrm{s}$. These low mud sound velocities result in a large reflection coefficient at the mud/water interface. This high acoustic reflectivity produces localized enhanced reflections, or bright spots, in amplitude.

In marine seismic operations, the water/air interface is a flat, strong reflector, with a reflection coefficient close to -1 . Additionally, the water/bottom interface is also a strong reflector. This situation produces an energy trap between these two boundaries. A pulse generated in the trap, or entering the trap from below, will be successively reflected between the two interfaces, with a time interval equal to the twoway travel time, and an amplitude decay dependent on the reflection coefficients. As 
a result, valid primary reflections from depth are obscured by previously established reverberations. In other words, interference between the upgoing and downgoing reflections results in the ringing seismograms typical of fluid covered areas. Sheriff and Geldart (1995) mention that two kinds of boundary conditions can produce this situation:

- The impedance contrast is so great that the reflection coefficient is very large, nearly unity. This situation can be observed in Lake Maracaibo where the reflection coefficient is close to -0.90 . Where this is observed, a polarity reverses.

- Waves within the waveguide are incident on the boundary at an angle greater than the critical angle, so that total reflection occurs and little energy leaks through the boundary. In Lake Maracaibo, the critical angle between the fluid and the mud layer, with gassy sediments, is between $12^{\circ}$ and $28^{\circ}$.

A common example of the first case can be observed in the interface between the fluid layer and the surface of the fluid layer. The second case can be observed in the interface between the fluid layer and the sea floor. Normal mode propagation in the water layer produces seismograms showing waves which could easily be mistaken for a long series of reflections from deep horizons. In Lake Maracaibo, in the zone without and with gassy sediments, the compressional record shows many multiples and ringing of the initial strong reflection due to these two situations (Levin, 1962). Figure 3.7 shows this situation in Lake Maracaibo.

The reverberation problem can be observed between $3 \mathrm{~s}$ and $4 \mathrm{~s}$ in the seismic line, which may at first give the erroneous impression that these are regular reflection records. Further examination shows that almost every detail of trace movement is repeated period after period for this window of time. This line is located in the area without gassy sediments problems. Additionally, it is possible to observe in Figure 3.7 low frequency and low velocity ground roll, less than $14 \mathrm{~Hz}$ and $700 \mathrm{~m} / \mathrm{s}$ respectively, ambient noise often in burst or spikes, low dominant frequency less than $30 \mathrm{~Hz}$, and industrial noise mostly from crew boats or the recording vessel. This boat noise consists of broad band direct arrivals at the near offset, of which the high frequency component persists over longer distances.

With the guided waves, in the general case, for most of the angles, there is destructive interference between the different waves. However, for certain angles, 




Fig. 3.7. Shot gather from Lake Maracaibo. This seismic section is from the area without gassy sediment in the mud layer. Multiple reflections can be observed between $3 s$ and $4 s$, due to the reverberation produced by the bottom and the surface of the lake. 
there is constructive interference and consequently a strong buildup of energy reflected at these angles (Sheriff and Geldart, 1995). Additionally, the compressional wave propagation in shallow water is strongly dependent upon the depth and compressional wave speed profile. If the profile has a negative gradient from the top to the bottom, propagation is particularly dependent upon the bottom configuration, and bottom and sub-bottom properties. This is because rays are refracted toward the bottom. If the profile has a positive gradient, surface roughness becomes the important factor.

Burg et al. (1951) mention that the high frequency of first energy arrivals associated with long range direct waves in shallow water comprises one manifestation in the guided waves'identification. Seismic observations reveal that this initial high frequency wave marks the beginning of a band of waves whose frequency rapidly decreases. These waves of continuously lower frequency persist until terminated by the air phase and these reflections involve rays whose angle of incidence at the bottom lies between the critical angle and the grazing angle. In this way, repetitive arrivals of waves, bouncing back and forth at different angles, can be observed in these circumstances. In order to explain this situation, in Figure 3.8 a shot point source at $\left(o, d_{1}\right)$ is considered in a coordinate system $(x, z)$, with $z=0$ representing the surface of the water and $z=h$ representing the level bottom.

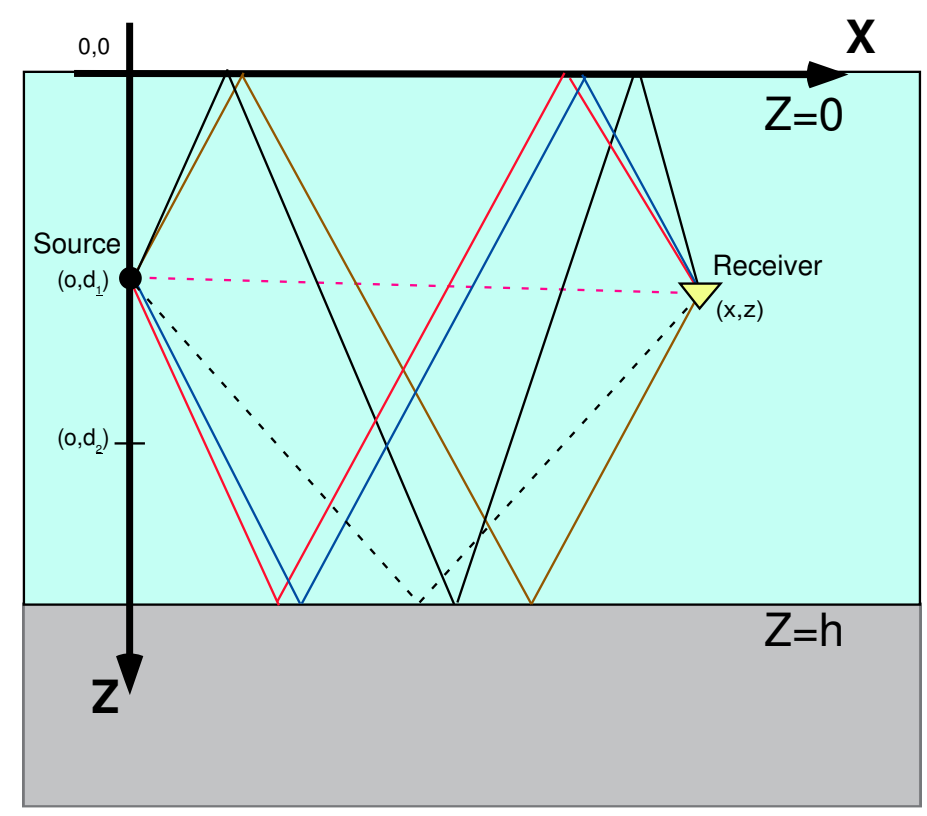

Fig. 3.8. Different types of ray paths from the source $\left(o, d_{1}\right)$ to $(x, z)$. These waves are bouncing back and forth within the layer because of nearly perfect reflectivity of the boundaries. 
Along $x=o$, a vertical line through the shot point, normal incidence applies upon the reflection of the waves at the boundaries of the water layer. It follows from the law of reflection that through successive reverberations the ray paths of the waves are retraced repeatedly. A particular point $\left(o, d_{2}\right)$ receives the wave alternately from above and below until energy attenuates. Another point $(x, z)$ is also struck by a succession of wave arrivals from a single explosion, but the mechanics are somewhat different. In this way, the different paths of waves provide multiple arrivals in direct relation to the different number of reflections off the free surface. Successive ray paths to $(x, z)$ are indicated in Figure 3.8. In this case, when the source and receiver are at some depth below the surface, it is possible to find at least four different rays for each order of bottom-reflected rays.

Burg et al. (1951) mention that if $n$ denotes the number of reflections a given ray experiences off the top surface, it is apparent that the angle the ray makes with the vertical can be associated with the discrete number $n$ and can be defined with equation (3.1):

$$
\theta_{n}=\sin ^{-1}\left(\frac{x}{v t}\right)=\tan ^{-1}\left(\frac{x}{2 m h-d_{1}+z}\right)
$$

where $v$ is the constant velocity of propagation, $t$ is the travel time and $\theta_{n}$ is the incident angle. In this case, $v t$ represents the path length. Now, it is generally assumed that the lower boundary condition is characterized by a compressional speed and medium density increase moving from water into the sediments. In this case, a critical angle exists so that the reflection coefficient is unity for angles from grazing up to the critical angle, and the phase shift decreases in a regular manner from $180^{\circ}$ at grazing to $0^{0}$ at the critical angle. From the critical angle up to normal incidence, the reflectivity decreases from one to a finite value, while the phase shift remains zero.

The condition at the surface is described by the free surface boundary condition, for which reflection is total and the phase shift is $180^{\circ}$ at all angles. In this way, the basic form of the propagation depends on the compressional and shear wave speed structure. Therefore, three different profiles can be considered: the isovelocity profile, the negative gradient profile and the positive gradient profile. In Lake Maracaibo the best profile that fits the model of the structures found in this area is a negative gradient profile. 
For the case of a negative compressional and shear wave speed gradient, no single ray type can propagate indefinitely because each type of ray has a limiting ray beyond which that ray type cannot be found. This establishes the long range limit for a given ray type. The short range limit for a given type may be assumed to coincide with the propagation of the critical ray of that ray type.

Another seismic feature in Lake Maracaibo is the effect produced by the gassy sediment observed in the mud layer. Thus, one of the most widely noted acoustic indications of a gassy sediment layer in the lake floor is the total loss of acoustic returns from deeper interfaces for a segment of the sub-bottom lake floor profile. From the viewpoint of the Lake Maracaibo exploration geophysicist, the attenuation and ringing of the compressional wave are probably the principal phenomena associated with low signal/noise ratio in the seismic data.

Levin (1962) mentions that if Wood's equation for the compressional wave velocity in mixtures is valid for a gas mud complex, it is possible to note that as little as one part per thousand by volume of gassy sediment will drop the velocity from $1500 \mathrm{~m} / \mathrm{s}$ to $500 \mathrm{~m} / \mathrm{s}$. However, Bourbie et al. (1987) mention that the compressional and shear wave velocities are relatively unaffected by the presence of gas for saturations ranging from $5 \%$ to $95 \%$, and therefore constitute relatively poor saturation indicators. By contrast, acoustic attenuations are highly sensitive to the different saturations.

The negative reflection coefficient, observed in the gassy sediments zone, implies phase inversion, and this means that a compressional wave is reflected as a rarefraction. However, this situation varied from place to place in the lake, as is evident in Figure 2.9. In this figure it is possible to observe that the seismic data has less frequency, and the signal/noise ratio decreases from Line No. 1 to Line No. 2 and from the Line No. 1 to Line No. 3 due to the seismic acquisition located in different places, with different features.

The gas saturations for Lake Maracaibo range between $2 \%$ to $0.2 \%$. Studies by Sarmiento and Kirby (1962) show that the mud layer contained enough gas to produce compressional wave speeds between $700 \mathrm{~m} / \mathrm{s}$ and $300 \mathrm{~m} / \mathrm{s}$, with estimations for the shear wave speeds between $200 \mathrm{~m} / \mathrm{s}$ and $600 \mathrm{~m} / \mathrm{s}$. However, the velocity of the compressional and shear wave, in the mud layer, increased with depth, suggesting that the gassy sediment concentration decreases with depth. The compressional wave speed in the water is about $1500 \mathrm{~m} / \mathrm{s}$ with an average density of $1.10 \mathrm{~g} / \mathrm{cc}$, producing with the low mud compressional speed a large reflection coefficient at the mud/water 
interface. Levin (1962) mentions that the reflection coefficient in Lake Maracaibo is between -0.8 to -0.7 in normal incident.

In Line No. 1 the seismic acquisition was located in the area without gassy sediment in the mud layer, but for the Line No. 2 and Line No. 3, the seismic acquisition was located in the area with gassy sediment in the mud layer. Levin (1962) mentions another phenomenon which may be related to the features of the layers configuration. When charges were colocated and detonated into the mud layer, a low frequency and a low velocity wave was generated. This wave was dispersive with a group velocity lower than the phase velocity and with lower frequencies corresponding to group velocity. The phase velocity shows a range between $800 \mathrm{~m} / \mathrm{s}$ and $625 \mathrm{~m} / \mathrm{s}$ and in general, drops as the frequency increases. The group velocity was of the order of $330 \mathrm{~m} / \mathrm{s}$. Levin (1962) states that the mud wave frequency generated depended most strongly on the position of the shot relative to the layer being detonated because the velocity of the compressional and shear wave in the mud layer increased with depth.

This work shows that the ringing and the attenuation, along with other equally important phenomena, due to the gassy sediment located in the mud layer, reduces the seismic data quality, and is a function of the gassy sediment concentration and the mud layer thickness. 


\section{CHAPTER IV}

\section{SEISMIC MODELING}

A synthetic seismogram is the result of one of many of different forms of forward modeling used to predict the seismic response of the earth. Synthetic seismograms are computed by assuming a mathematical model with a particular geometry of the source and velocity layering that approximates an elastic or anelastic earth.

Solutions of varying approximations to the wave equation in the geometry of the model result in theoretical amplitude versus time of arrival times, or wavefield seismograms. These may be used for studying wave propagation through various media, calculating amplitude and waveform responses to various sources in different models and comparison with observed seismograms to improve the model. The calculation of synthetic seismograms normally requires a solution to the wave equation for the geometry and characteristic parameters of the model. This entails application of appropriate boundary conditions exactly or numerically.

Models can be developed to address problems of structure and stratigraphy prior to acquisition of seismic data and during the interpretation of the data. Sheriff and Geldart (1995) mention that agreement between data and a model does not prove that the model is correct since there can be numerous models that agree with a given data set.

Important to seismic exploration and research are programs for creating synthetic data. Such programs find their use both in the practical problem of modeling real data, as well as in the testing of new processing programs. A processing program that will not work on idealized model data will likely not work on real seismic data. It is also important to note that many seismic processing algorithms used in modeling programs may be viewed as inverse processes. The first step in such an inverse problem may be to create a method to solve the forward problem, and then formulate the solution to the inverse problem as a back propagation of the recorded data to its position in the subsurface.

In order to understand the mud layer problem in Lake Maracaibo, several synthetic seismograms will be built with different layers configurations, velocities, thicknesses, etc. In this way, the information obtained from these models will be compared 
to the field seismic data from the lake and the different parameters will be changed in order to find the best match. In this section, it will shown that the zone without gassy sediments is very different in attenuation, lithology, thickness, etc. from the zone with gassy sediments in Lake Maracaibo.

\subsection{Modeling Without Gassy Sediment}

It is well known that the bottom sediments of Lake Maracaibo are loosely deposited and consist mainly of extremely fine grained sediments. The median diameter of the particles that form these sediments is close to $1 \mu$ and porosity averages $80 \%$. These sediments form a mud layer that is a few meters thick over much of the northwestern portion of the lake. The thickness increases rapidly southeastward to between $30 \mathrm{~m}$ and $40 \mathrm{~m}$. The mud layer is $20 \mathrm{~m}$ thick over much of the central deep and eastern areas. These sediments rest upon an uneven surface formed by a tough, mottled, silty clay, which generally shows brownish or yellowish iron oxide colorations (Sarmiento and Kirby, 1962). This mud layer has a density between $1.2 \mathrm{~g} / \mathrm{cc}$ and $1.3 \mathrm{~g} / \mathrm{cc}$. The compressional wave velocity of the lake mud layer varies from $1420 \mathrm{~m} / \mathrm{s}$ to $1600 \mathrm{~m} / \mathrm{s}$ in the area without gassy sediment.

In order to understand which condition(s) cause poor quality seismic data, synthetic seismograms were built to attempt to model the situation in Lake Maracaibo. The first step was constructing models of Lake Maracaibo that represent the mud layer zone characteristics, with a good approximation of the velocities, thicknesses, densities, etc., that can reproduce a good estimation of the problems observed in Figure 4.1. Figure 4.2 shows the frequency spectrum for Line No. 1. In this case, only the central traces were taken into consideration in the frequency spectrum analysis.

The central frequency is close to $30 \mathrm{~Hz}$. The seismic data set shown in Figure 4.1 has a basic processing that was applied to this seismic section. In this case, the Seismic Unix library, SU, was used and the processing applied was the following (Stockwell and Cohen, 2000):

- A bandpass filter with parameters equal to 2-4-50-65. The main function was limited to the bandwidth of the output signal in the low and high frequency components.

In order to build the synthetic seismogram to attempt to model the features of Lake Maracaibo, several parameters have to be considered. Therefore, different 


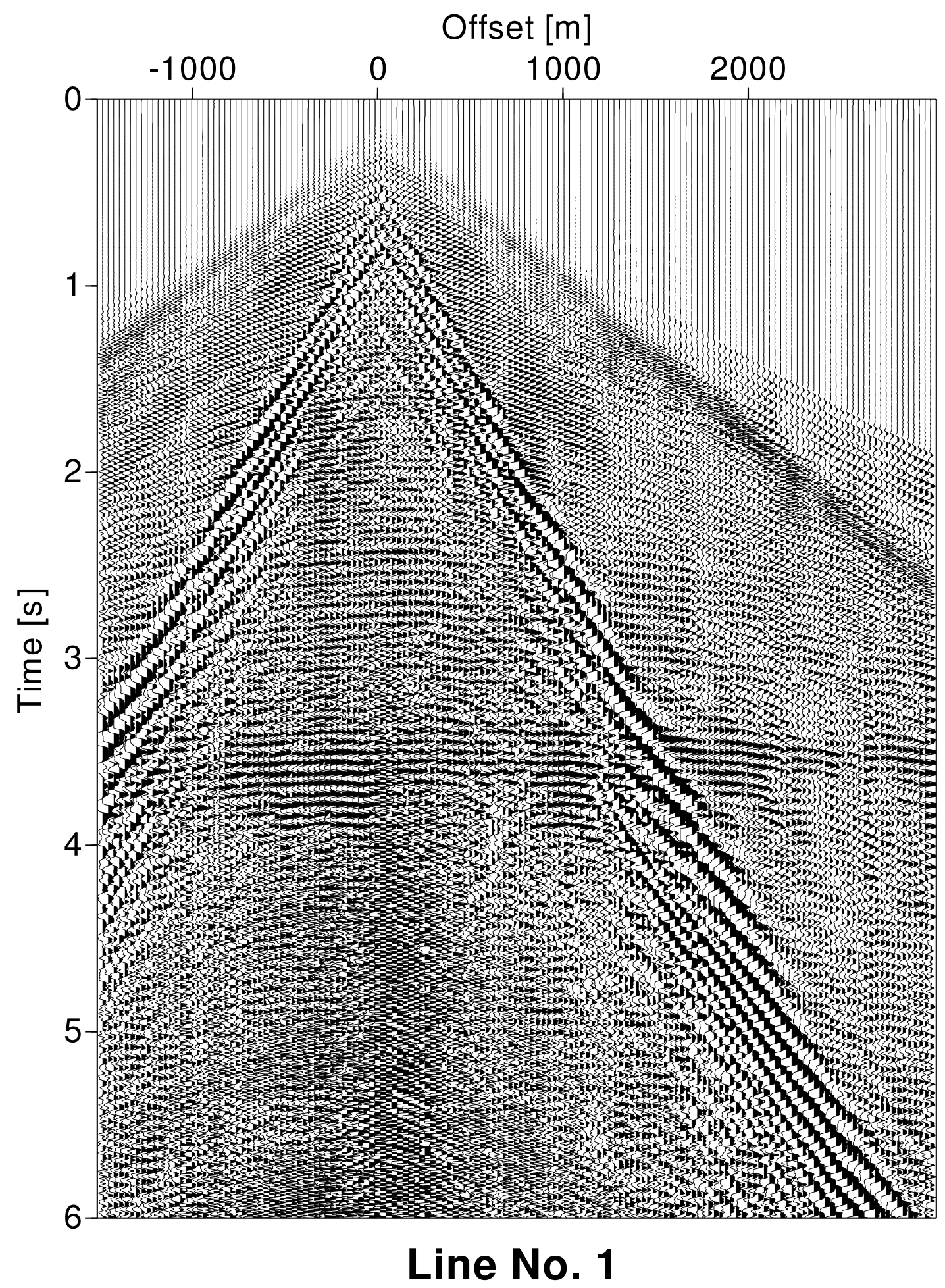

Fig. 4.1. Shot gather from Lake Maracaibo. This seismic section is from the area without gassy sediment in the mud layer. A basic processing was applied to the seismic data section that involved bandpass filter. 


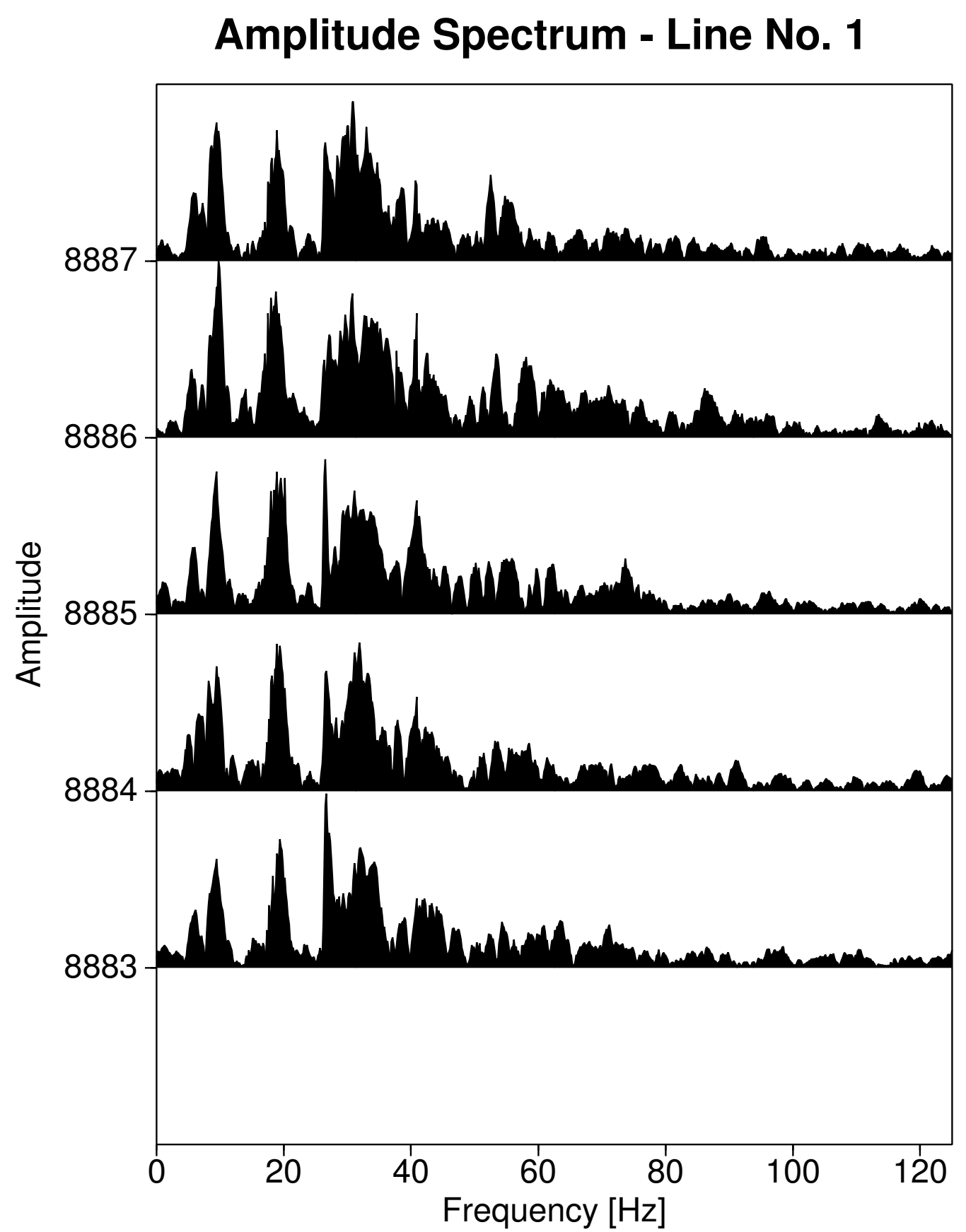

Fig. 4.2. Frequency spectrum for the raw data, Line No. 1. The results show that the central frequency is close to $30 \mathrm{~Hz}$. However, in the synthetic seismogram $25 \mathrm{~Hz}$ was used as the central frequency. 
numbers of layers, velocities, thickness distributions and attenuation factors will be used. Layers that try to simulate the viscous losses, solid friction losses, mud layers without gassy sediment with low shear wave speed between layers, and hard sediment below the mud layer will be used.

Probably the most important factor to be considered is the velocity function for this area, together with the depth of the different reflectors that are observed in the raw data, see Figure 4.1. The velocity function was established through the velocity analysis made with the raw data. In this case, the seismic section from Lake Maracaibo, without gassy sediment in the mud layer, was used.

Figure 4.3 shows both the common depth point (CDP) gather from the area without gassy sediment in the mud layer and the stacking velocity semblance for CDP gathers. A bandpass filter with parameters 7-15-55-70 was applied.

In the stacking velocity semblance for CDP gathers, the amplitudes are summed together along different hyperbolas described by the travel time equation. The summation is carried out for different values of time and velocity, and the result is contoured in the time-velocity space.

The correct values of the velocity semblance analysis will describe a hyperbola that coincides with the reflection event to give a large summation value. This characteristic can be observed between $2 \mathrm{~s}$ and $4 \mathrm{~s}$ in the CPD gather from Lake Maracaibo in Figure 4.3; otherwise, the summation values are small. This situation can be observed between $0 \mathrm{~s}$ and $2 \mathrm{~s}$ in the CDP gather, see Figure 4.3.

The depth of the different reflectors observed in the seismic data set, between $2 \mathrm{~s}$ and $4 \mathrm{~s}$, was estimated using well information from different locations in Lake Maracaibo. This was calibrated by trial and error through the construction of several synthetic seismograms. Figures 4.4 and 4.5 show a layer configuration used to represent the first $100 \mathrm{~m}$ and the deeper reflector in the Lake Maracaibo zone. These layers attempt to simulate the viscous losses, solid friction losses and mud layer effect, without gassy sediment with low shear velocities and hard sediment below the mud layer. To establish the parameters in the mud layer zone without gassy sediment, several synthetic seismograms with different attenuation factors and distinct velocity distributions will be utilized without changing the numbers of layers and thickness distributions. In this situation, the attenuation quality factor, $Q_{\alpha}$ and $Q_{\beta}$, used in the viscous losses medium was between 100 to 150 and 10 to 20, respectively. In the solid friction losses and the mud layer medium, the the attenuation quality factor, $Q_{\alpha}$ 

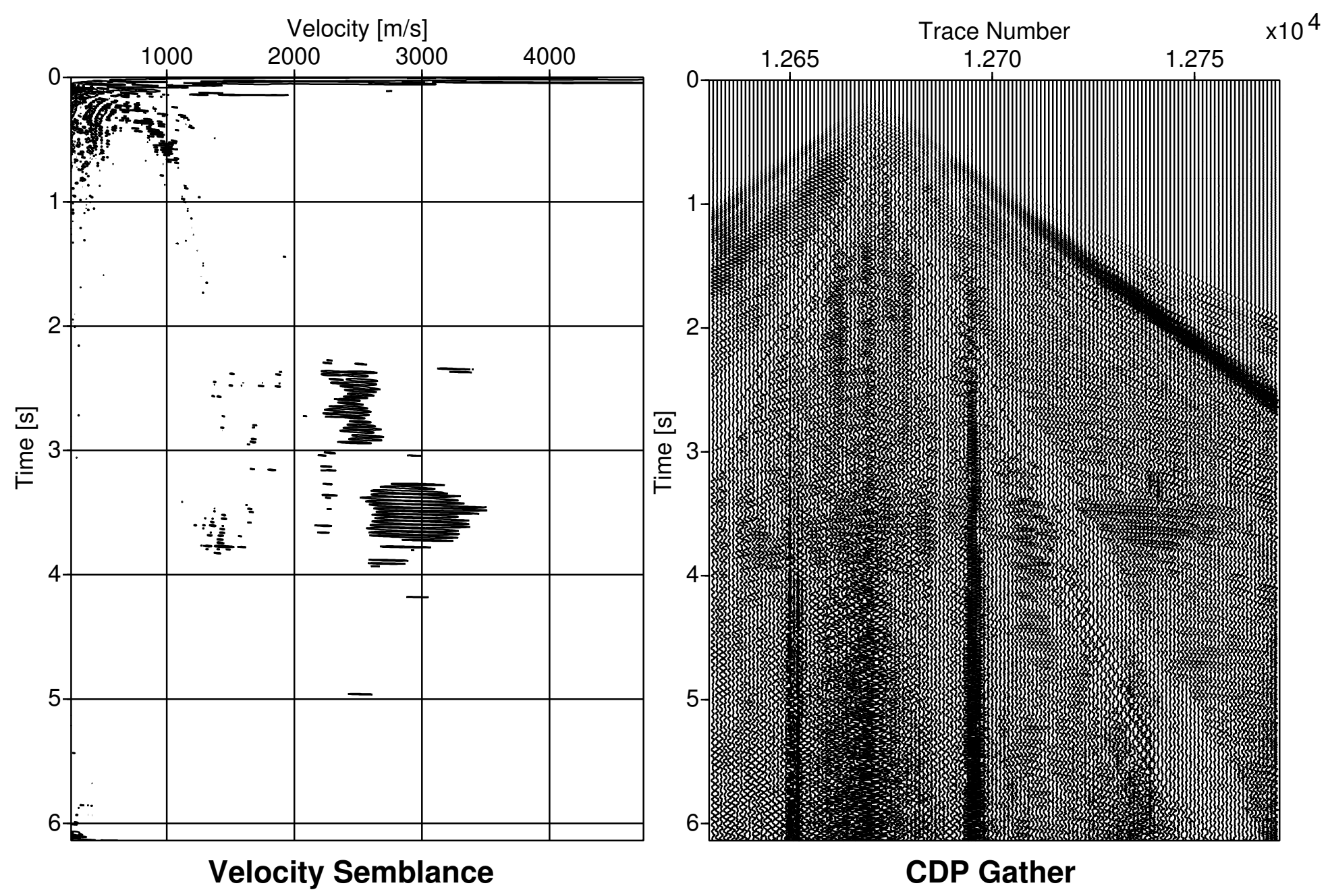

Fig. 4.3. CDP gather and velocity analysis made over the seismic section, from Lake Maracaibo, without gassy sediment in the mud layer. In this analysis the SU library was used. Only velocity information between $2 \mathrm{~s}$ and $4 \mathrm{~s}$ was available 


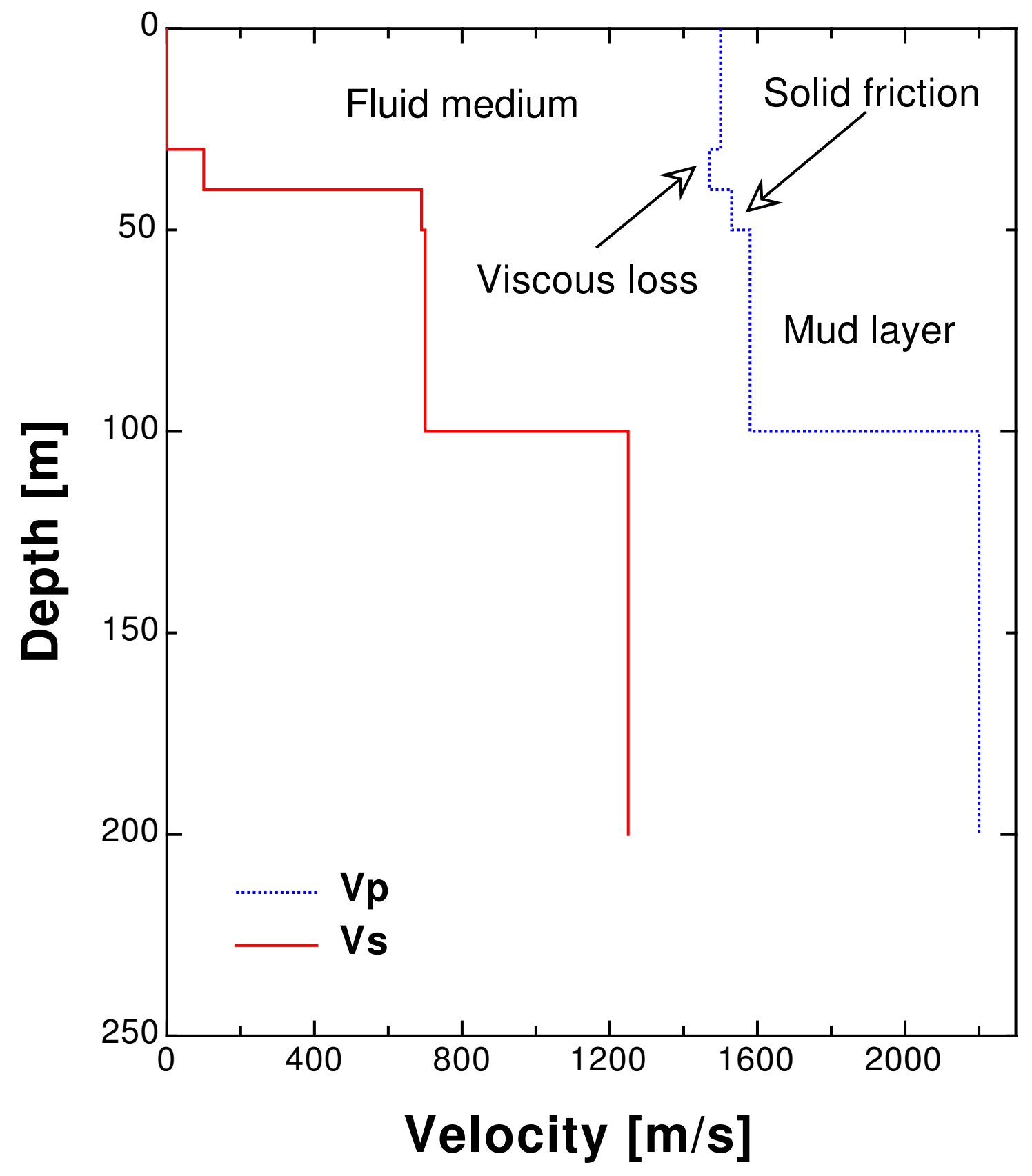

Fig. 4.4. Velocity profile used to build the synthetic seismogram in Lake Maracaibo for the mud layer zone. Three different kinds of attenuation mechanisms are considered: viscous losses, solid friction losses and mud layer effect, without gassy sediment. This measures only the first $100 \mathrm{~m}$. 


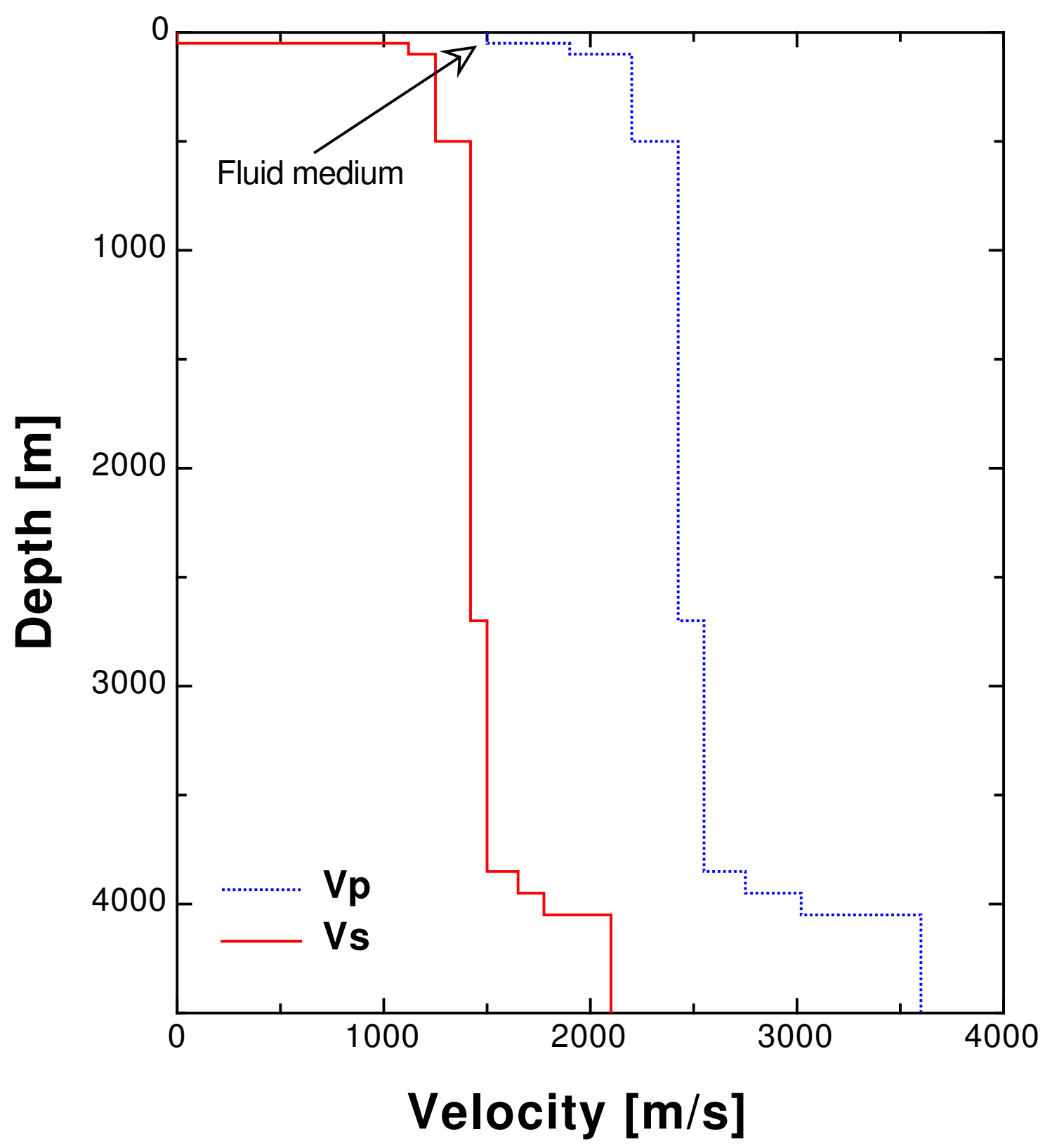

Fig. 4.5. Velocity profile used to build the synthetic seismogram in Lake Maracaibo for the mud layer zone. This configuration is for the deeper reflector. This configuration was used for both situations, the mud layer without gassy sediment and with gassy sediment, respectively. 
and $Q_{\beta}$, used was between 100 to 150 and 90 to 120, respectively (Lay and Wallace, 1995; Hamilton, 1972; Bowles, 1997).

Table 4.1 shows the relationship between the quality factor $Q$, for the compressional and shear wave, and the sediment types. These values were used to both control the accuracy in the synthetic seismogram built and improve the fit between the real data and the mathematical model. These values are just for the seismic frequency range between $10 \mathrm{~Hz}$ and $150 \mathrm{~Hz}$.

Table 4.1. Relationships between compressional wave quality factor $Q_{\alpha}$ and shear wave quality factor $Q_{\beta}$ versus sediment types. This relationship was used to control the quality factor $Q$ used in the different synthetic seismograms (Lay and Wallace, 1995; Hamilton, 1972; Bowles, 1997).

\begin{tabular}{|c||c||c|}
\hline Sediment type & $Q_{\alpha}$ & $Q_{\beta}$ \\
\hline \hline Mud & $30-150$ & $5-120$ \\
\hline Shale & $28-32$ & $8-12$ \\
\hline Sandstone & $56-60$ & $29-33$ \\
\hline Sand medium & $31-35$ & $13-16$ \\
\hline Sand very fine & $29-32$ & $12-14$ \\
\hline Gassy sediment & $5-90$ & $200-\infty$ \\
\hline
\end{tabular}

In Lake Maracaibo the attenuation of seismic data, in the zone without the gassy sediment problem, is due to the viscous losses between the particles and the fluid, the solid friction losses between the particles, and the mud layer effect. For the compressional and shear waves, the velocity can be observed to rise as the frequency increases. This can be explained by the fact that the inertial forces increase simultaneously. Indeed, these inertial forces, which are different for the fluid and the solid part, generate a differential movement between the fluid and the fluid/solid combination, and other combinations, due to permeability effects (Bourbie et al., 1987).

Hamilton (1980) mentions that different studies indicate an approximate first order dependence of attenuation coefficient $\alpha$ on frequency between a few $\mathrm{Hz}$ and several $\mathrm{Hz}$ in unconsolidated sediment and consolidated sediment, in sand, slit and mud for the compressional and shear waves in the frequency range between $15 \mathrm{~Hz}$ and $90 \mathrm{~Hz}$.

Stoll and Bryan (1969) found that attenuation coefficient $\alpha$, in unconsolidated 
sediment and consolidated sediment, is controlled by the frame losses, solid friction, at low frequency, resulting in a linear dependence on frequency. This study showed a first order dependence of attenuation on frequency for both compressional and shear waves. This analysis was made in the frequency range between $40 \mathrm{~Hz}$ and $390 \mathrm{~Hz}$.

Hamilton (1980) mentions that for computations and predictions of compressional and shear wave energy losses in natural, poorly sorted sands, silt-clays, mudstone, shale, unconsolidated sediment and consolidated sediment, it is recommended that attenuation of shear and compressional waves be considered to vary as the first power of frequency. This recommendation was followed in the modeling of the different synthetic seismograms built from the mud layer with gassy sediment and without gassy sediment. Additionally, studies with the attenuation coefficient $\alpha$ independent of frequency were made.

Several synthetic seismograms were built with the configuration described in Figure 4.4 and the seismic geometry shown in Figure 4.6. A split spread seismic configuration using 150 receivers per shot, with a separation of $30 \mathrm{~m}$ between receivers, and offset minimum equal to $15 \mathrm{~m}$ was used. The source depth was $5 \mathrm{~m}$ and the receivers depth was $15 \mathrm{~m}$.

The main objective, with this synthetic seismogram, was to verify if the configuration, shown in Figure 4.4, can be applied to Lake Maracaibo for the mud layer zone without gassy sediment and where viscous losses, solid friction and the mud layer effect are possible attenuation mechanisms.

The synthetic seismograms were built using several programs, which employed the reflectivity method to solve the $2 \mathrm{D}$ full wave equation for the general layered anisotropy medium. In the reflectivity method, the amplitudes of the waves propagating in all the layers are solved by one large system of equations. A point source of spherical waves is located in any position in a plane parallel-layered system, specified by a wider parameter list for each layer. The results of this methodology describe multiple reflections of any order. Therefore, the spherical waves developed by the source at constant frequency are represented by an equivalent set of plane waves (Mandal and Mitchell, 1986).

The different results obtained with the modeling, using the layer configuration described in Figure 4.4, were compared with the seismic data set shown in Figure 4.1, which is from Lake Maracaibo without gassy sediment in the mud layer. In this way, several synthetic seismograms were built with different parameters for: com- 


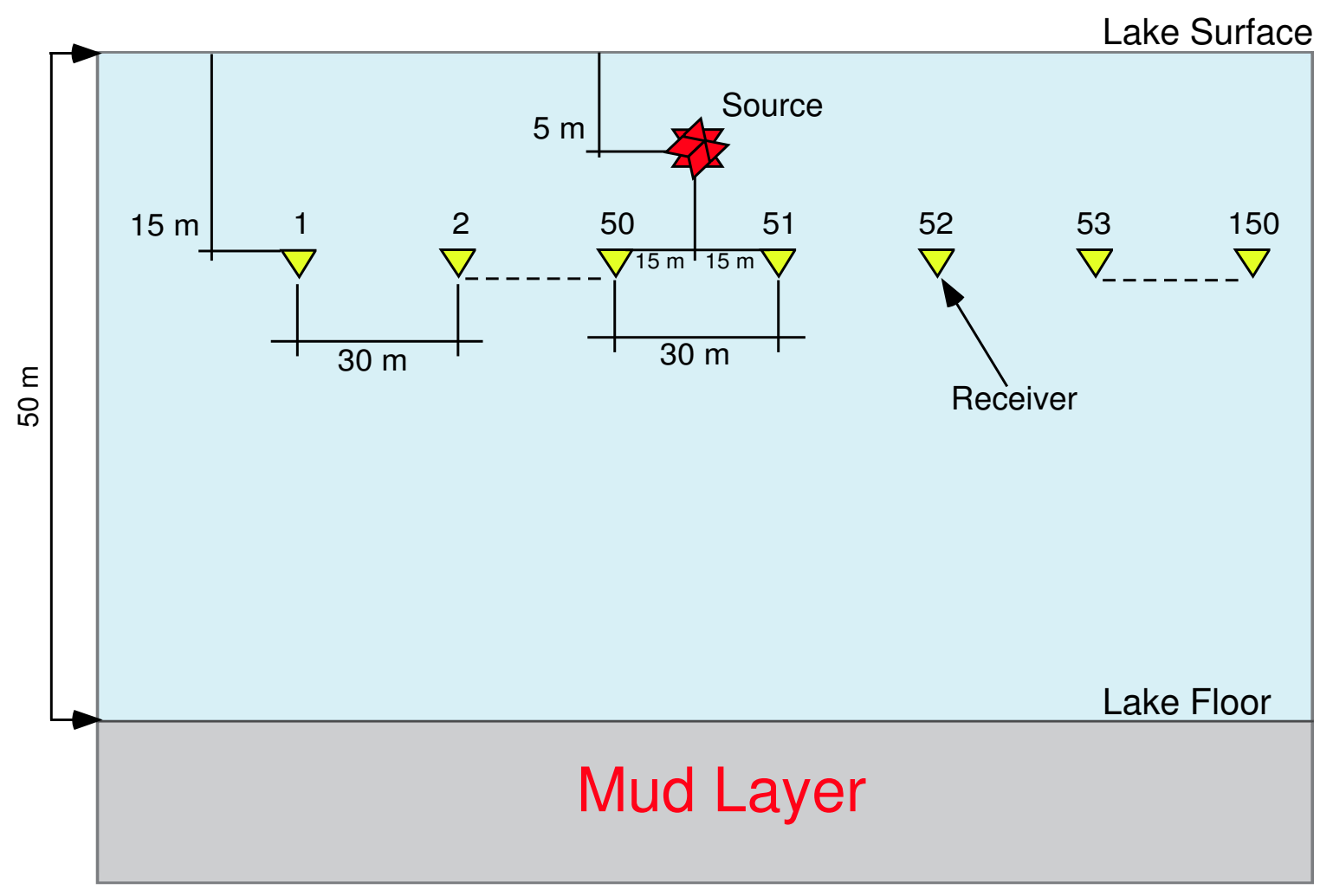

Fig. 4.6. Seismic acquisition configuration used to build the synthetic seismogram in Lake Maracaibo. This configuration was used in both cases; mud layer with the gassy sediment problem and without the gassy sediment problem, respectively. In this case, 150 receivers were used. An exaggerated vertical scale was used.

pressional and shear waves velocities, densities, thicknesses and quality factors, and were compared with the real data.

In order to find the best match between the synthetic seismogram and the seismic data set from Lake Maracaibo, several changes were made in the parameters described above. In this way, the match between the synthetic seismogram and the seismic data set was improved. Therefore, several combinations of compressional and shear waves velocities, densities, thicknesses and attenuation quality factors were used. This process was refined by trial and error.

In order to understand the attenuation effect over the seismic data from the zone without gassy sediment, several seismograms were compared to the already existing $2 \mathrm{D}$ seismic data to determine if the attenuation factor(s) is related to the poor quality data, due to the high signal/noise ratio, which can be observed in the raw data from this area. Therefore, several attenuation values and combinations of these parameters 
were used in the first $100 \mathrm{~m}$ of Lake Maracaibo. The best match, between the seismic data set shown in Figure 4.1 and the synthetic seismogram, is shown in Figure 4.7.

The most important factor considered in building the synthetic seismogram was the shear wave velocity that can be observed in the viscous losses and solid friction medium respectively, because these two media have enough rigidity to transmit shear wave. The shear wave is important, in water saturated media, because compressional waves can be partially converted to shear waves, and the energy is rapidly attenuated.

In the water saturated medium, it is possible to observe that there is little rigid elastic structure and the sediment particles primarily move in the sound field. Therefore, the velocity behavior will follow that of a suspension. It appears when a sediment has a concentration of mineral particles greater than 23\% (Hamilton et al., 1955). At this concentration of mineral particles, the sediment assumes a measurable rigidity. Its Poisson's ratio becomes lower than the value of 0.50 for fluids, and its compressibility is less than that of a more fluid mixture. In these cases the compressional wave is partially converted to shear wave.

It is possible to appreciate, in Figure 4.8, several similarities between the seismic data set from Lake Maracaibo and the synthetic seismogram. There are at least six different coincident points.

1. Seismic reflection: Seismic reflection is the return of some energy to the earth's surface from rock boundaries. Seismic refraction is the bending of waves as they pass from one rock layer to another. Both provide information about the earth's internal layers. This reflection is due to the layer located $2700 \mathrm{~m}$ in the subsurface. In this case, it is possible to observe the ringing produced for the fluid layer. Different multiples are produced for the different layers located in the real data and the model.

2. Seismic reflection: These reflections are produced by the layers between $3850 \mathrm{~m}$ to $4050 \mathrm{~m}$ in the subsurface. In this case, it is possible to observe the ringing produced by the fluid layer. The other different multiples are produced for the different layers.

3. Stoneley wave: This is a type of seismic wave propagated along an interface. This wave is always possible at solid/fluid interfaces. The Stoneley wave is of varying frequency, which depends on the elastic properties of the rock. This 




Fig. 4.7. Synthetic seismogram for Lake Maracaibo obtained using a layer configuration without gassy sediment and the seismic geometry acquisition split spread. The model configuration was composed with 13 layers. 

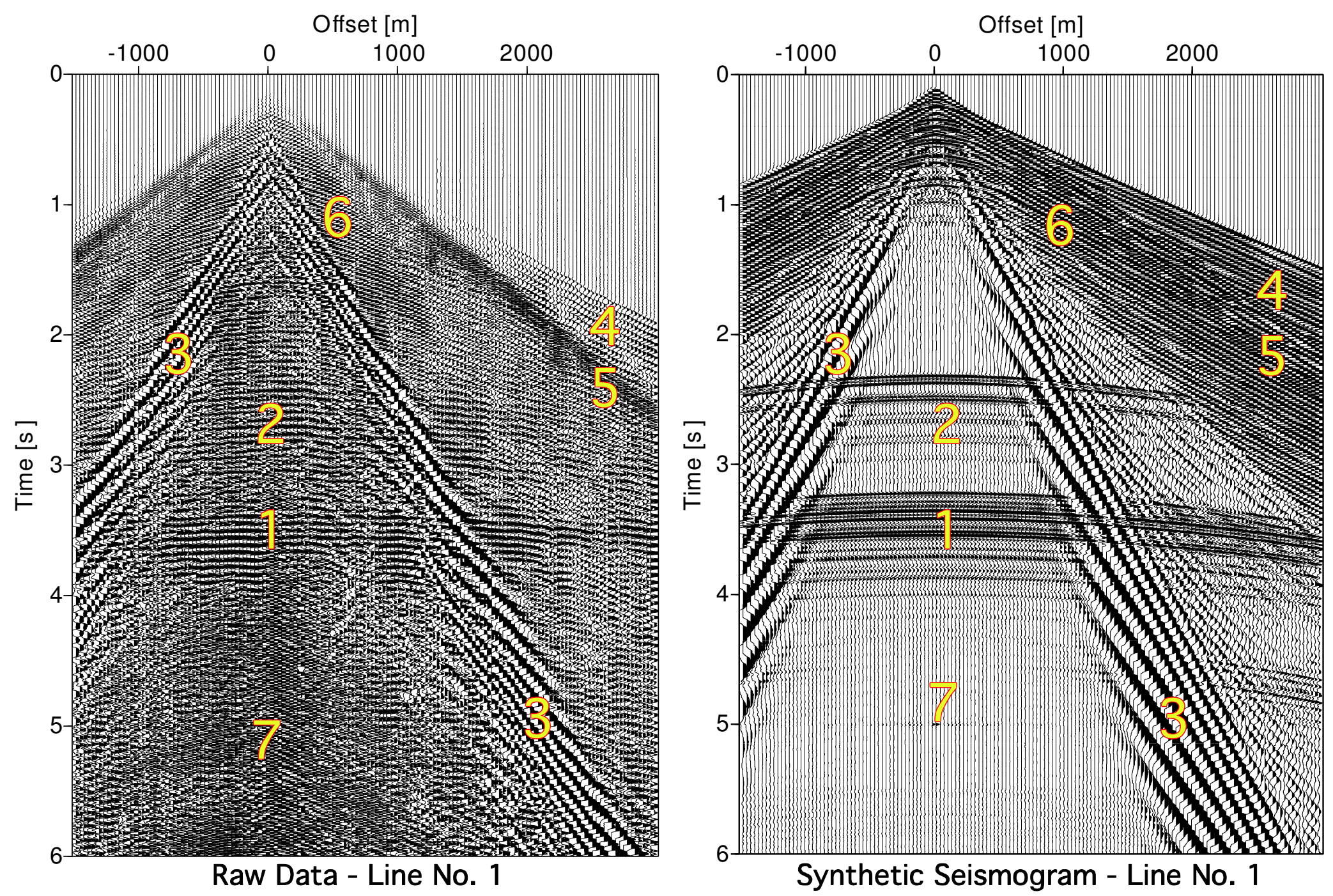

Fig. 4.8. Seismic data set from Lake Maracaibo in the mud layer area without gassy sediment and the synthetic seismogram built for this zone. There are at least six similarities. 
wave also has high amplitude, and its velocity is close to that of the compressional wave in fluid, or the shear wave in water saturated medium. This wave is produced by converting compressional wave to shear wave in the viscous losses and solid friction medium. For the case of a fluid/solid interface, the Stoneley wave always exists. This wave shows appreciable dispersion.

4. Head wave: This is a wave entering a relatively high velocity medium whose incident and refracted angle is the critical angle. Theoretically, it is a ray that moves away from a source along a horizontal interface until it encounters an irregularity, from which it reflects and returns toward the source. This event provides an easy velocity estimate. In Lake Maracaibo, the critical angle is between $30^{\circ}$ and $40^{\circ}$ in this zone.

5. Direct waves [water break]: The direct wave is the portion of the wave that travels from source to geophone directly by the shortest path. The arrival of the energy travels in the water directly from the the source to the detector. This wave shows appreciable dispersion, which is due to the strong vertical gradient that can be observed in this zone.

6. Spatial aliasing: This is defined as the distortion that is produced by sampling a signal below its Nyquist rate. Spatial aliasing means insufficient sampling of the data along the space axis. In this case, the spatial aliasing is due to the sampling interval between receivers, $30 \mathrm{~m}$.

7. Ambient noise and/or industrial noise: This refers to the background or random earth movements not related to the source. The noise is divided into coherent noise and random noise. The synthetic seismogram does not show this noise because it was not taken into account. However, it is possible to observe a small amount of numerical noise.

From the above analysis comparing the seismic data from Lake Maracaibo and the synthetic seismogram, it is possible to understand that three different attenuation mechanisms are present in the zone with the mud layer without gassy sediment. The first is the viscous losses between the particles and the fluid. The second relates to the solid friction losses between the particles. The third is due to the mud layer. In the mud layer case, the attenuation mechanism is similar to what is observed in the solid friction. 
With this modeling, it is possible to observe converted shear wave from the compressional wave in the viscous losses, solid friction and mud layer medium. This converted compressional energy is rapidly attenuated, but produces the Stoneley wave with a slow apparent velocity. The final phenomenon, observed in this area, is the ringing produced by the water layer, which can be observed in both the raw data and the synthetic seismogram.

The ringing occurs because the water/bottom interface has a very strong reflection coefficient and small critical angle. In this case, the ringing involves rays whose angles of incidence at bottom lie between the critical angle and the grazing angle. This ringing is easily observed in the deeper reflections, between $2 \mathrm{~s}$ and $3 \mathrm{~s}$, and between $3 \mathrm{~s}$ and $4 \mathrm{~s}$. Table 4.2 shows the different parameters used in the building of the synthetic seismograms in the mud layer zone without gassy sediments.

Table 4.2. The different parameters used in the building of the synthetic seismograms in the mud layer zone without gassy sediments in Lake Maracaibo.

\begin{tabular}{|c||c||c||c||c||c||c|}
\hline Layer & $Q_{\alpha}$ & $Q_{\beta}$ & $V_{p}[\mathrm{~m} / \mathrm{s}]$ & $V_{s}[\mathrm{~m} / \mathrm{s}]$ & $\rho[\mathrm{g} / \mathrm{cc}]$ & Thickness $[\mathrm{m}]$ \\
\hline \hline Fluid medium & 200 & - & 1500 & 0 & 1.10 & 30 \\
\hline Viscous medium & 110 & 20 & 1470 & 100 & 1.25 & 10 \\
\hline Solid friction medium & 90 & 80 & 1550 & 690 & 1.30 & 10 \\
\hline Mud layer & 90 & 80 & 1580 & 700 & 1.32 & 50 \\
\hline Milagro & 160 & 120 & 2200 & 1250 & 1.70 & 400 \\
\hline Layer A & 160 & 120 & 2425 & 1420 & 2.20 & 2200 \\
\hline Layer B & 160 & 120 & 2550 & 1500 & 2.30 & 1150 \\
\hline Layer C & 160 & 120 & 2750 & 1650 & 2.50 & 200 \\
\hline Layer D & 160 & 120 & 3020 & 1775 & 2.60 & 200 \\
\hline Layer E & 160 & 120 & 3600 & 2100 & 2.65 & Half space \\
\hline
\end{tabular}

In this case, the results show that the best match between the raw data and the synthetic seismogram was when the attenuation quality factor $Q$ was independent of frequency, in the frequency range between $1 \mathrm{~Hz}$ and $70 \mathrm{~Hz}$. When the value of $n$ was different than 0, the synthetic seismogram showed unrealistic results. Figure 4.9 shows two synthetic seismograms with the same layer configuration, velocities, thicknesses, 

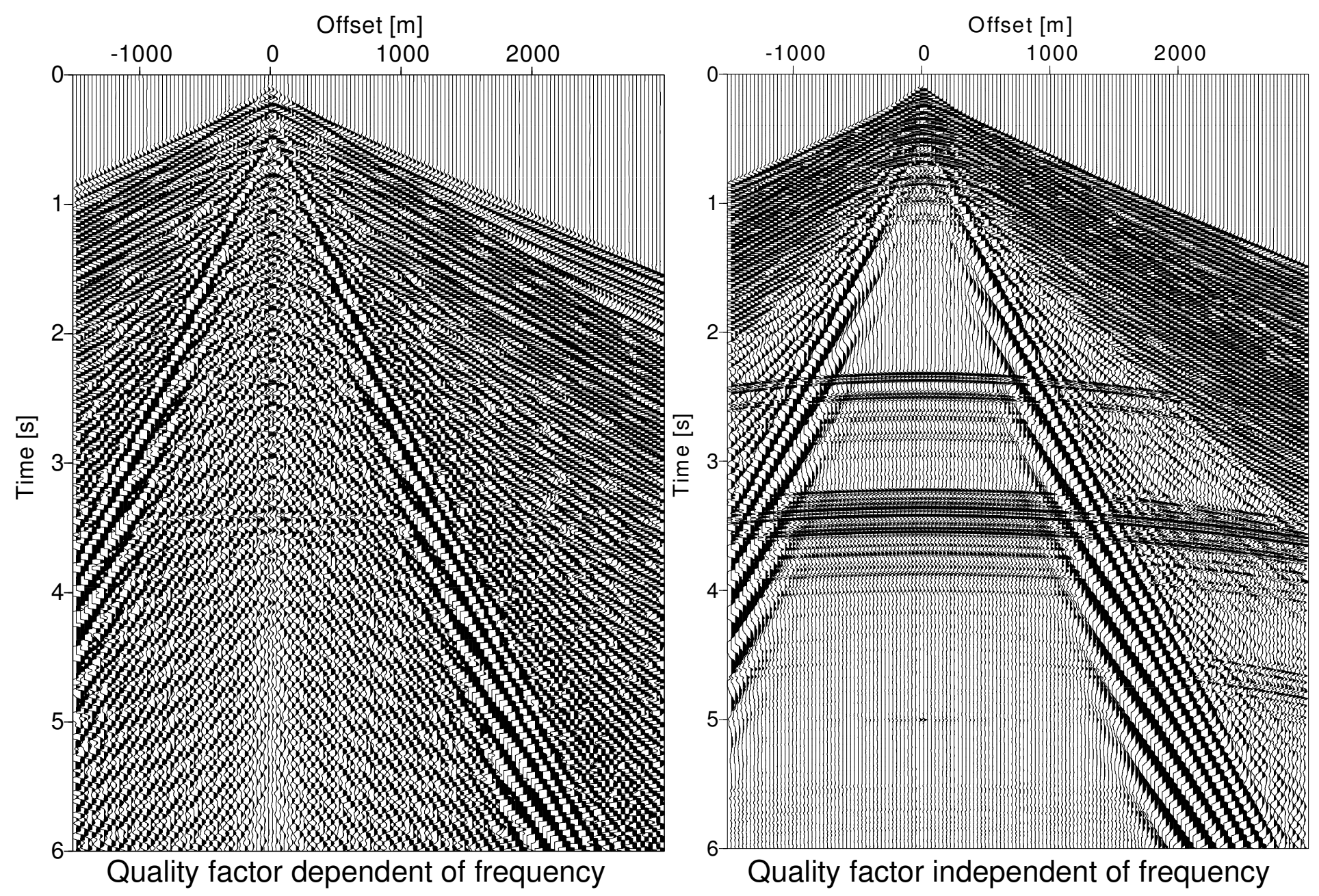

Quality factor independent of frequency

Fig. 4.9. Synthetic seismogram with the same layer configuration, velocities, thicknesses, densities, etc., but with different $n$ value, in the zone without gassy sediments. In the figure on the right, the value for $n$ was 1 ; on the left, the value for $n$ was 0. 
etc., but different $n$ values. It is possible to appreciate that when the $n$ value is different than 0 the results are unrealistic. Equation (4.1) shows the expression used in the build of the synthetic seismograms. This situation could be observed for $n$ values of 1 and 2. Figure 4.10 shows a synthetic seismogram built for the mud layer zone without gassy sediments, but with a different attenuation quality factor $Q_{\beta}$ than in the Figure 4.8 for the viscous losses zone. In this case, the $Q_{\beta}$ value was equal to 100. Therefore, when an appropiate value for the attenuation is not considered in the attenuation estimation, an unrealistic result is obtained.

$$
Q(f)=Q_{o} f^{n}
$$

where $Q(f)$ is the attenuation quality factor as a function of frequency, $Q_{o}$ is the initial attenuation quality factor value, $f$ is the frequency dependence and gives the dependence of the attenuation upon the frequency as a funtion of $n$.

\subsection{Modeling With Gassy Sediment}

Gasses found in sediments include carbon dioxide, hydrogen sulfide, ethane, nitrogen and methane. In Lake Maracaibo only the methane and carbon dioxide are found in considerable quantities. All the gasses originate from biogenic or petrogenic processes. Biogenic gas is derived from bacterial activity primarily within the top few meters of sediment. In areas of rapid sedimentation, gas accumulations can be buried to depths well below those at which they were generated. This is the situation observed in Lake Maracaibo. Petrogenic gas is produced from organic precursors at high temperatures and pressures, and consequently normally exists at depths greater than $1000 \mathrm{~m}$.

In Lake Maracaibo the gas in the lake bed is in solution in the pore water and undissolved in the form of gas-filled voids, which is more common. Gas in the pore water will have little effect on the physical properties of the lake bed unless the ambient pressures are reduced. When this occurs, the gas in the water increases the compressibility several orders of magnitude above that in gas-free water, thereby greatly reducing the velocity and increasing the attenuation of acoustic waves. However, this situation has not been observed in Lake Maracaibo. Undissolved gas affects the acoustic properties of the lake bed due to the compressibility, gas quantity, etc. In this situation, the boundary between fluid and gassy sediment horizons represents 


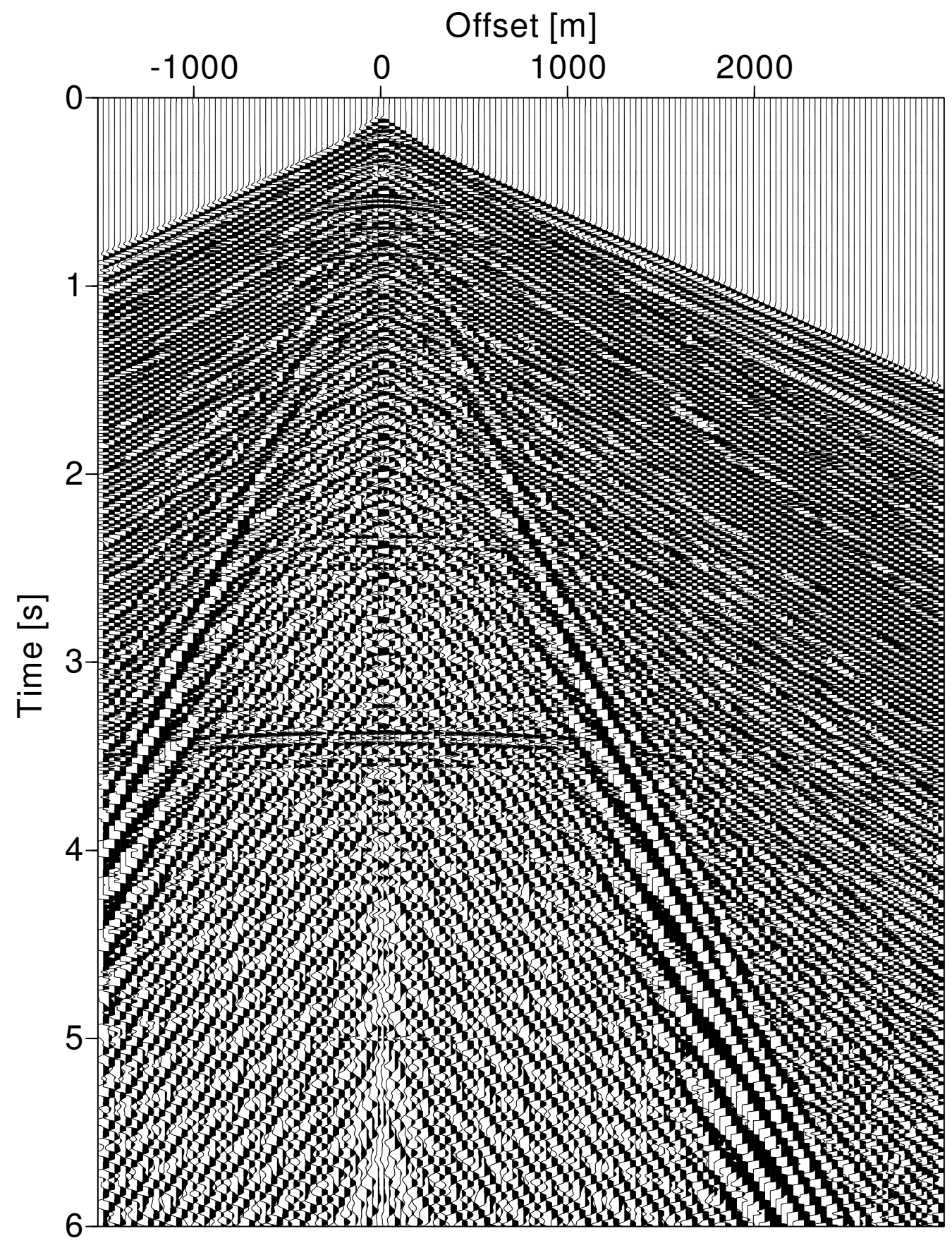

Fig. 4.10. Synthetic seismogram, in the zone without gassy sediments, with the same layer configuration, velocities, thicknesses, densities, etc., but with the attenuation quality factor $Q_{\beta}$ equal to 100 in the viscous losses zone. 
a significant acoustic impedance mismatch. High acoustic reflectivity is associated with such an impedance, in seismic sections.

Although other impedance contrasts can also cause enhanced reflections, a phase reversal of the reflected pressure wave, where this enhanced reflection can be observed, narrows the interpretation. It is a strong indication of probable free gas in the reflecting horizon (Anderson and Bryant, 1990). However, this feature is not present in the available seismic data set from Lake Maracaibo. Because the compressional wave velocity is reduced, at least, by an order of magnitude from the water saturated lake floor value, a very small critical angle is produced in Lake Maracaibo. This value is between $12^{\circ}$ and $28^{\circ}$.

As a result, most of the energy of a downward propagating acoustic wave will be reflected by the gassy sediment layer. The seismic record shows this by many multiples of the initial strong reflection from the gassy sediment layer. This ringing is seen in the seismic section from Lake Maracaibo with the gassy sediment layer, see Figures 4.11 and 4.13. However, this ringing cannot be appreciated easily in the seismic section with gassy sediments because this area has low frequency content and a very low signal/noise ratio due to the attenuation produced by the gassy sediment in the mud layer.

Figure 4.11 and Figure 4.12 show the raw data and the frequency spectrum from the Line No. 2. Figure 4.13 and Figure 4.14 show the raw data and the frequency spectrum from the Line No. 3. In this case, the frequency spectrum analysis was made over the central traces in both lines. In both lines, several features are present: a small signal/noise ratio, Stoneley wave with velocity between $900 \mathrm{~m} / \mathrm{s}$ and $1200 \mathrm{~m} / \mathrm{s}$, the head wave with velocity between $1600 \mathrm{~m} / \mathrm{s}$ and $1950 \mathrm{~m} / \mathrm{s}$, and the ringing with low frequency content. Additionally, it is possible to observe the ambient noise, industrial noise, aliasing and low frequency in almost all the seismic sections.

The most important feature observed in these two seismic sections, Line No. 2 and Line No. 3, is the attenuation of the compressional wave due to the gassy sediment in the mud layer. This attenuation is greater by several orders of magnitude than the attenuation observed in the zone without gassy sediment in the mud layer due to the viscous losses, solid friction losses and mud layer effect. Levin (1962) mentions that the compressional wave velocity is reduced by the presence of gas from decaying organic matter, between $300 \mathrm{~m} / \mathrm{s}$ and $700 \mathrm{~m} / \mathrm{s}$ when shear wave velocity is between $300 \mathrm{~m} / \mathrm{s}$ and $600 \mathrm{~m} / \mathrm{s}$. However, studies made using the synthetic seismogram 
show that the compressional and shear velocities are between $800 \mathrm{~m} / \mathrm{s}$ to $1000 \mathrm{~m} / \mathrm{s}$, and $700 \mathrm{~m} / \mathrm{s}$ to $900 \mathrm{~m} / \mathrm{s}$ respectively.

Several attenuation definitions are available, and one of them is that the attenuation is based on energy loss per oscillation for a given travel time. In this case, the high frequencies undergo more oscillations than do the low frequencies, so the waves at higher frequencies are more attenuated, and wavelet shape changes with time.

Mechanisms for intrinsic $Q$ include absorption, grain sliding, viscous flow of pore fluid or gas, and perhaps viscous relaxation. Another phenomenon, scattering, as from intrabed multiples, has nearly the same effect as intrinsic $Q$ and can be included with the intrinsic $Q$ into an effective $Q$. It is well known that the attenuation may be attributed to either phenomenon, absorption or scattering, depending on the geological model. In this study, effective $Q$ and a $Q$ independent on frequency will be considered.

Few quantitative reports of $Q$ in gas sands exist, and even fewer in gassy mud. Some of the lowest $Q$ values reported for fine-grained marine sediment may have unknowingly incorporated some gas, but they were not reported as gas bearing. Using sandstones, $Q$ was measured over a range of partial saturations and frequencies, and showed at $100 \mathrm{~Hz}$, a decrease in $Q$ from 50 at $100 \%$ saturation, to 20 at $90 \%$ saturation and back to 50 as saturation decreases. This rather interesting local minimum of $Q$ at partial saturation was attributed to peak loss of energy from pore fluids moving relative to the surrounding matrix (Bourbie et al., 1987).

Others report similar values, $Q_{\alpha}$ ranging between 10 and 100, for dry, as well as saturated sandstones in the lab and unconsolidated sediment in the field. Now, for the compressional wave case, it is possible to expect $Q_{\alpha}$ for gassy sediment to be between 5 and 90. For the shear wave case, it is possible to expect $Q_{\beta}$ for gassy sediment to be larger than 200. This means that the shear wave propagation is not affected by the gassy sediment in the medium.

The ratio $V_{p} / V_{s}$ of the compressional wave velocity to the shear wave velocity corresponds one-to-one with Poisson's ratio. The Poisson's ratio of a material influences the transmission and reflection of stress waves, the decay of the stress with distance, and the distribution of stress around holes and cracks. Equation (4.2) shows the relationship between the Poisson's ratio and the compressional and shear wave 




Fig. 4.11. Shot gather from Lake Maracaibo. This seismic section is from the area with gassy sediment in the mud layer, Line No. 2. A basic processing was applied to the seismic data section that involved bandpass filter. 


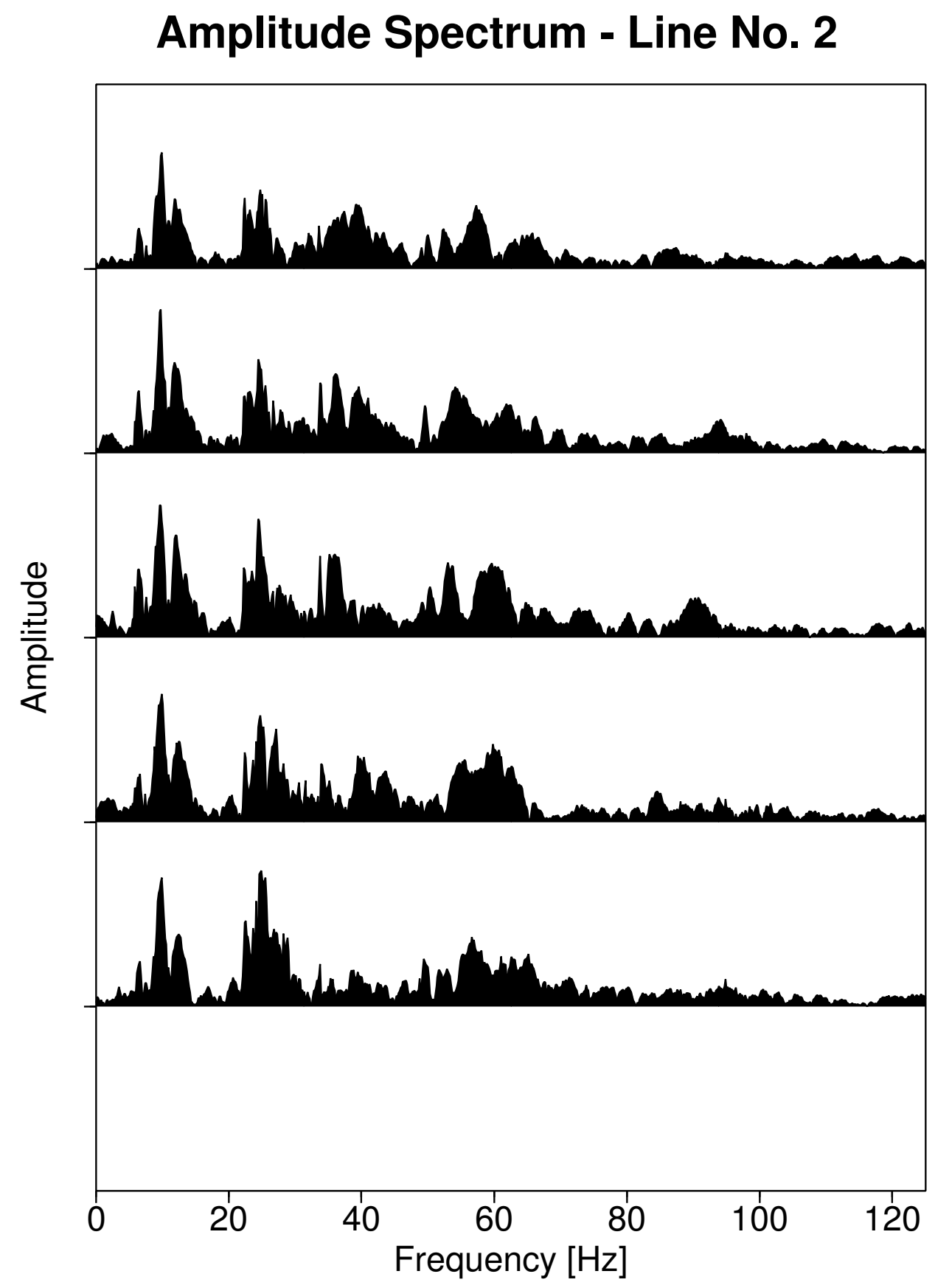

Fig. 4.12. Frequency spectrum for the raw data, Line No. 2. The results show that the central frequency is close to $25 \mathrm{~Hz}$. It is possible to observe the different frequency content between this line and Line No. 3. 


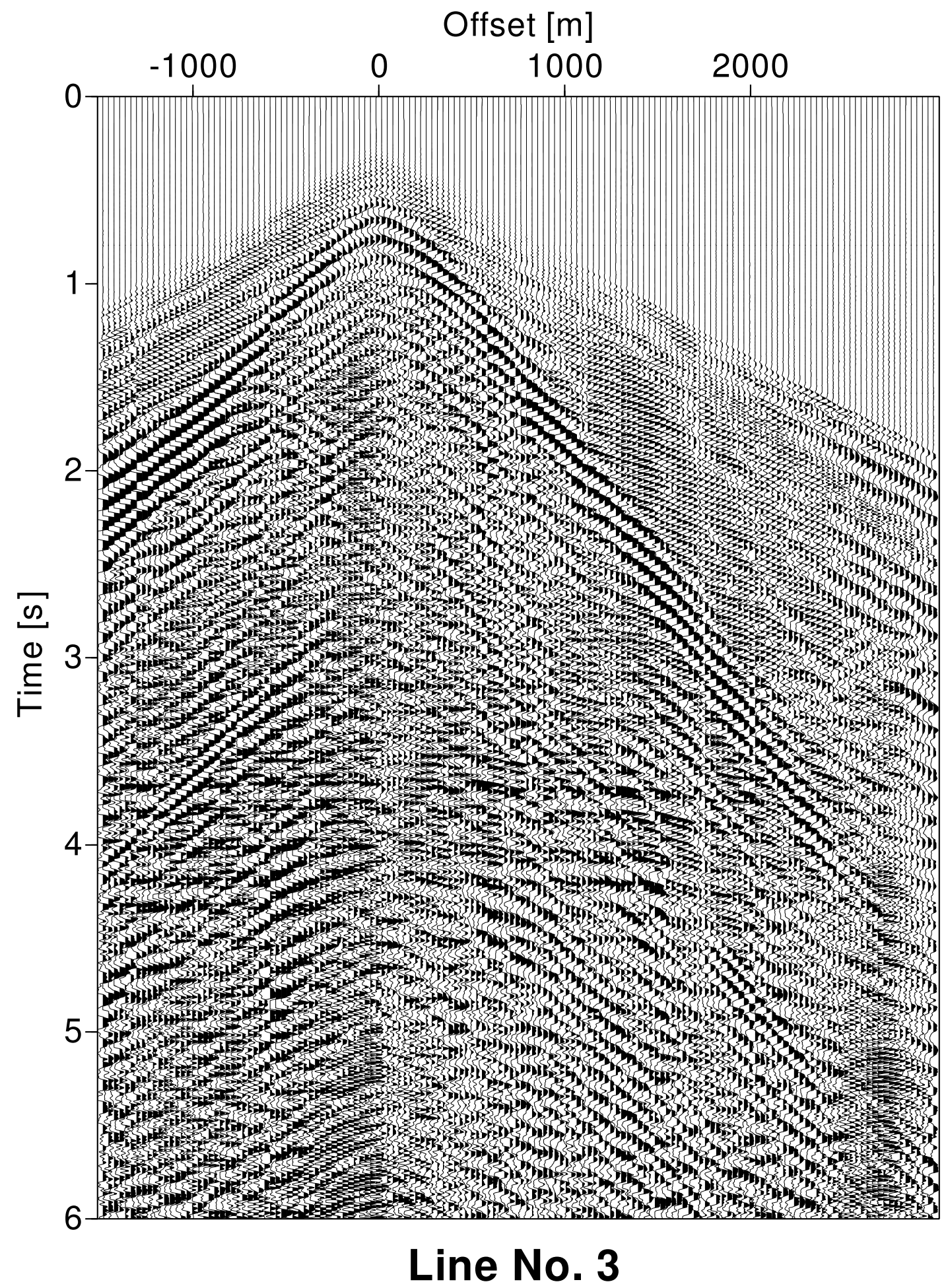

Fig. 4.13. Shot gather from Lake Maracaibo. This seismic section is from the area with gassy sediment in the mud layer, Line No. 3. A basic processing was applied to the seismic data section that involved bandpass filter. 


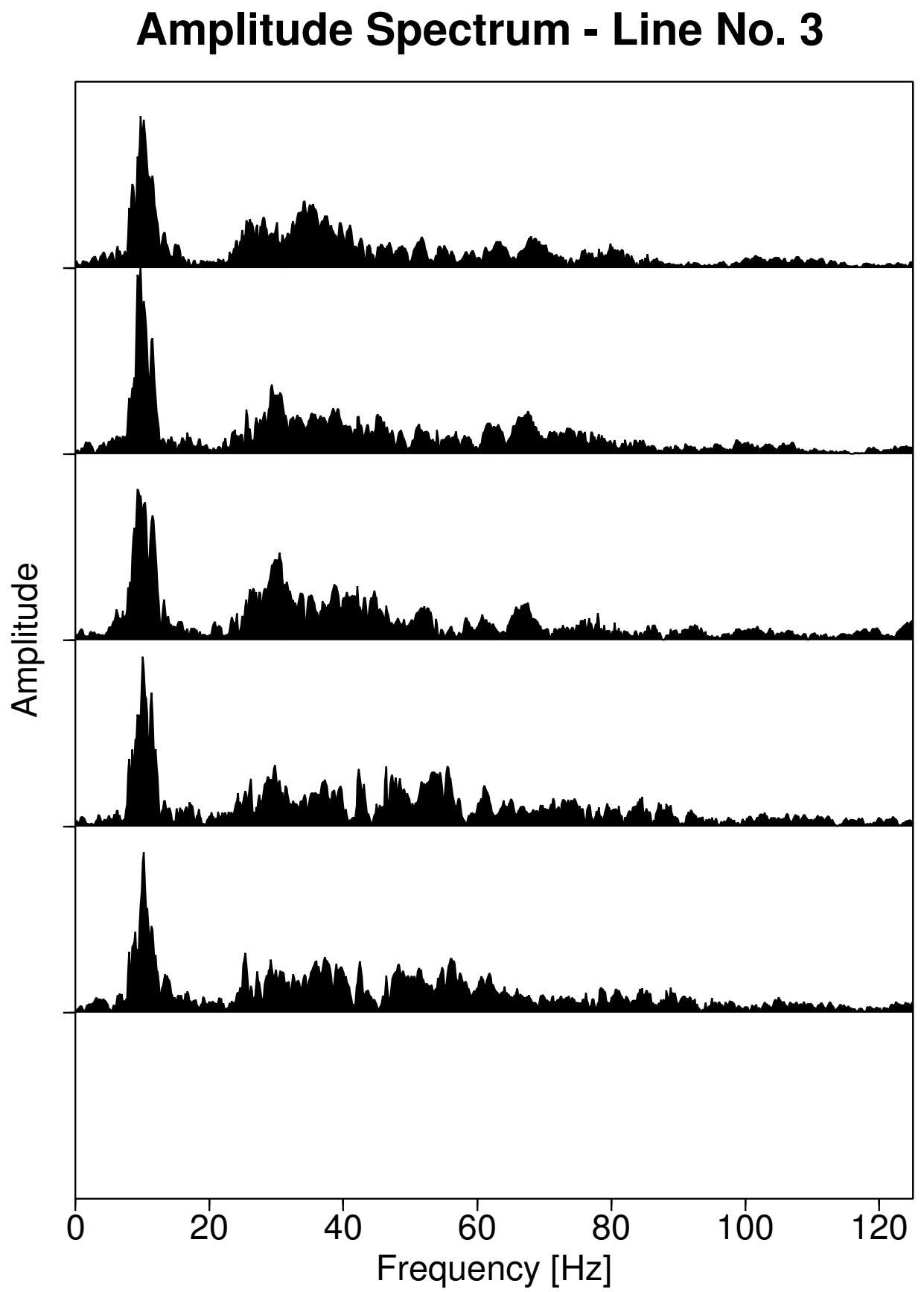

Fig. 4.14. Frequency spectrum for the raw data, Line No. 3. The results show that the central frequency is close to $15 \mathrm{~Hz}$. In the synthetic seismogram, the central frequency was $20 \mathrm{~Hz}$. 
velocities:

$$
\frac{V_{p}}{V_{s}}=\sqrt{\frac{2(1-\sigma)}{1-2 \sigma}},
$$

where $V_{p}$ is the compressional wave velocity, $V_{s}$ is the shear wave velocity and $\sigma$ represents the Poisson's ratio. High values of Poisson's ratio or a high $V p / V s$ ratio correspond to unconsolidated rocks. Compact rocks display Poisson's ratio between 0.2 and 0.35 , while gas sands have a very low Poisson's ratio, about 0.15 , or low $V p / V s$ ratio. In water-saturated sands, by contrast, Possion's ratio is about 0.4. In Figure 4.15, the foregoing correlations between the type of rock, saturation and Poisson's ratio are merely indicative values.

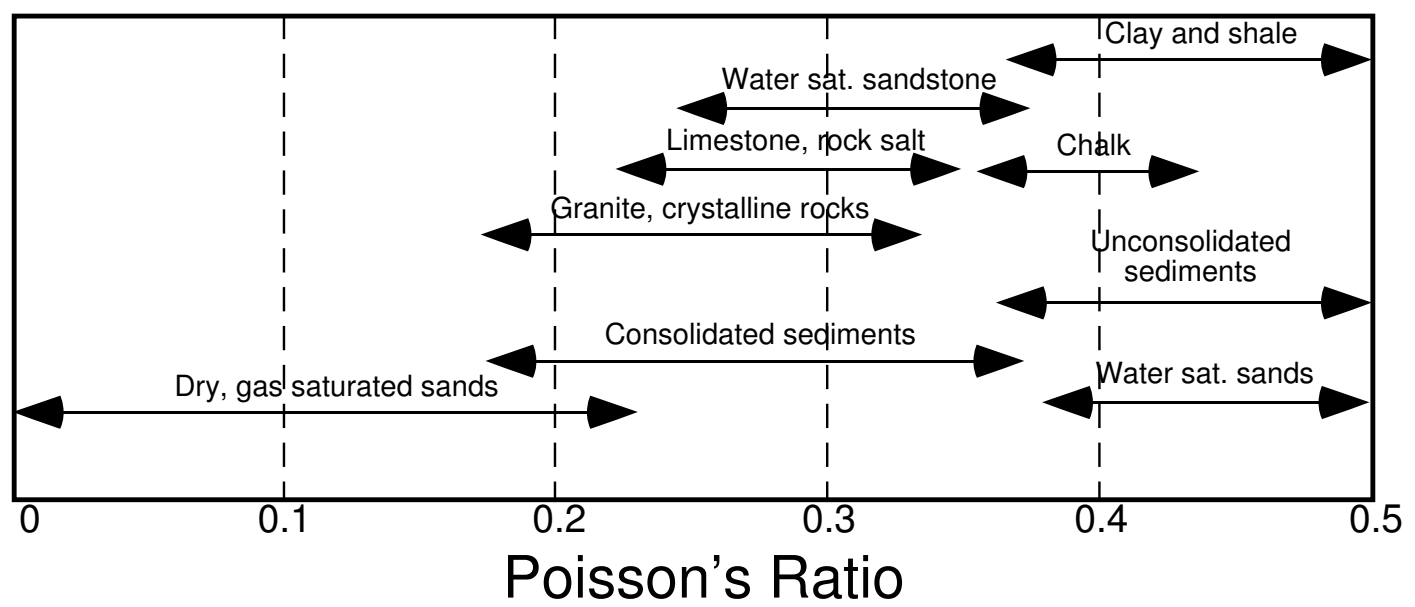

Fig. 4.15. Average Poisson's ratios for different lithologies. These ratio values were taken into account in the compressional wave and shear wave velocities selection in the synthetic seismograms (Bourbie et al., 1987).

The relationships between attenuation and other physical properties in unconsolidated sediment, consolidated sediment and gassy sediment are of considerable importance in determining the causes of attenuation and in selecting appropriate anelastic models. It is well known that the seismic velocities vary when a very small amount of gas is introduced into the system. Several studies show that the compressional wave and shear wave velocities are affected by the presence of gas for saturations ranging from $0.1 \%$ to $5 \%$, but these waves are relatively uneffected by the presence of gas for saturations between 10\% to 95\% (Bourbie et al., 1987; Domenico, 1976). Therefore the velocities constitute relatively poor saturations indicators. 
The most predominant characteristics in the mud layer zone in Lake Maracaibo with gassy sediment medium are the increases in the attenuation of the compressional wave and the decreases of the compressional and shear waves' velocities by several orders of magnitude, see Figure 3.6. Additionally, an increase in reflection amplitude, by a change in the medium elastic properties when gas replaces water in the medium, can be observed. This situation produces a large reflection coefficient at the mud/water interface and inverse polarity. The amplitude is not a linear function of the amount of gas present in the reservoir because no biunivocal relationship exists between seismic features, bright spots for example, and the presence of a gas in the medium. Because the water/air interface is a flat, strong reflector, with a reflection coefficient close to -1 , and the water/mud interface is also a strong reflector with a reflection coefficient between -0.7 and -0.8 , the wave energy is trapped, producing a strong quantity of ringing in this area. The ringing occurs within the fluid layer involving rays whose angle of incidence at the bottom lies between the critical angle and the grazing angle.

In order to verify and understand if the low signal/noise ratio in the seismic data set, observed in Figure 4.11 and Figure 4.13, is due to the gassy sediment in the mud layer zone, the model configuration, described in Figure 3.6, was used to build the different synthetic seismograms. Figure 4.16 is the final synthetic seismogram obtained after several iterations with different parameters: velocities, thicknesses, densities and layers distribution. The results show that the layer distribution in the mud layer zone with gassy sediment is different from the mud layer zone without gassy sediment.

The most important difference is the presence of a rigid shale layer embedded in the mud layer zone with gassy sediment, which is not evident in the mud layer zone without gassy sediment. This rigid shale layer is probably related to the substratum $C_{1}$ described by Sarmiento and Kirby (1962). Figure 3.5 shows a generalized stratigraphic column where it is possible to observe detailed lithological information from this area.

The best match between the seismic data set shown in Figure 4.13 and the synthetic seismogram built using the layers configuration and seismic geometry acquisition described in Figures 3.6 and 4.6 respectively, is shown in Figure 4.16. The results show that the small signal/noise ratio is due to the low compressional and shear waves' velocities in the gassy sediment localized immediately below the rigid 
shale layer. The Poisson's ratio for this layer is between 1.10 and 1.15 and the value of the attenuation quality factor $Q_{\alpha}$ generated for the gassy sediment layer is between 5 and 20. Figure 4.17 shows the relationship between the raw data and the synthetic seismogram from the mud layer zone with gassy sediment. Points of similarity are observed as follows:

1. Destructive Stoneley wave: This wave is generated as a combination of two different waves. One comes from the rigid shale layer embedded in the mud layer and the other is the wave generated in the gassy sediment. This wave has an apparent velocity between $1100 \mathrm{~m} / \mathrm{s}$ and $850 \mathrm{~m} / \mathrm{s}$. Destructive interference patterns that influence the motion of this wave can be observed.

2. Direct waves [water break]: This wave is traveling with the water velocity layer. In this case, there is appreciable dispersion. The phase velocity is between 1500 $\mathrm{m} / \mathrm{s}$ and $1200 \mathrm{~m} / \mathrm{s}$.

3. Ringing: The gassy sediment in the mud layer attenuated the high frequencies traveling through this medium. There is a high amount of ringing with low frequency content. Additionally, multiples produced for the different layers located in the real data and the model can be observed.

4. Spatial aliasing: This spatial aliasing is produced due to the sampling interval between receivers, in this case $30 \mathrm{~m}$.

5. Constructive Stonely wave: This wave is generated for the low velocity from the viscous loss layer, solid friction layer and the mud layer without gassy sediment. This wave has high dispersion, and the phase velocity is between $200 \mathrm{~m} / \mathrm{s}$ and $700 \mathrm{~m} / \mathrm{s}$.

6. Ambient noise and/or industrial noise: This feature cannot be observed in the synthetic seismogram because it was not taken in to account. However, it is possible to observe a small amount of numerical noise.

7. Head wave: This wave cannot be generated clearly in the raw data from the mud layer zone with gassy sediment. In the synthetic seismogram it is easily observable. In this case, the critical angle is less than $25^{\circ}$. 


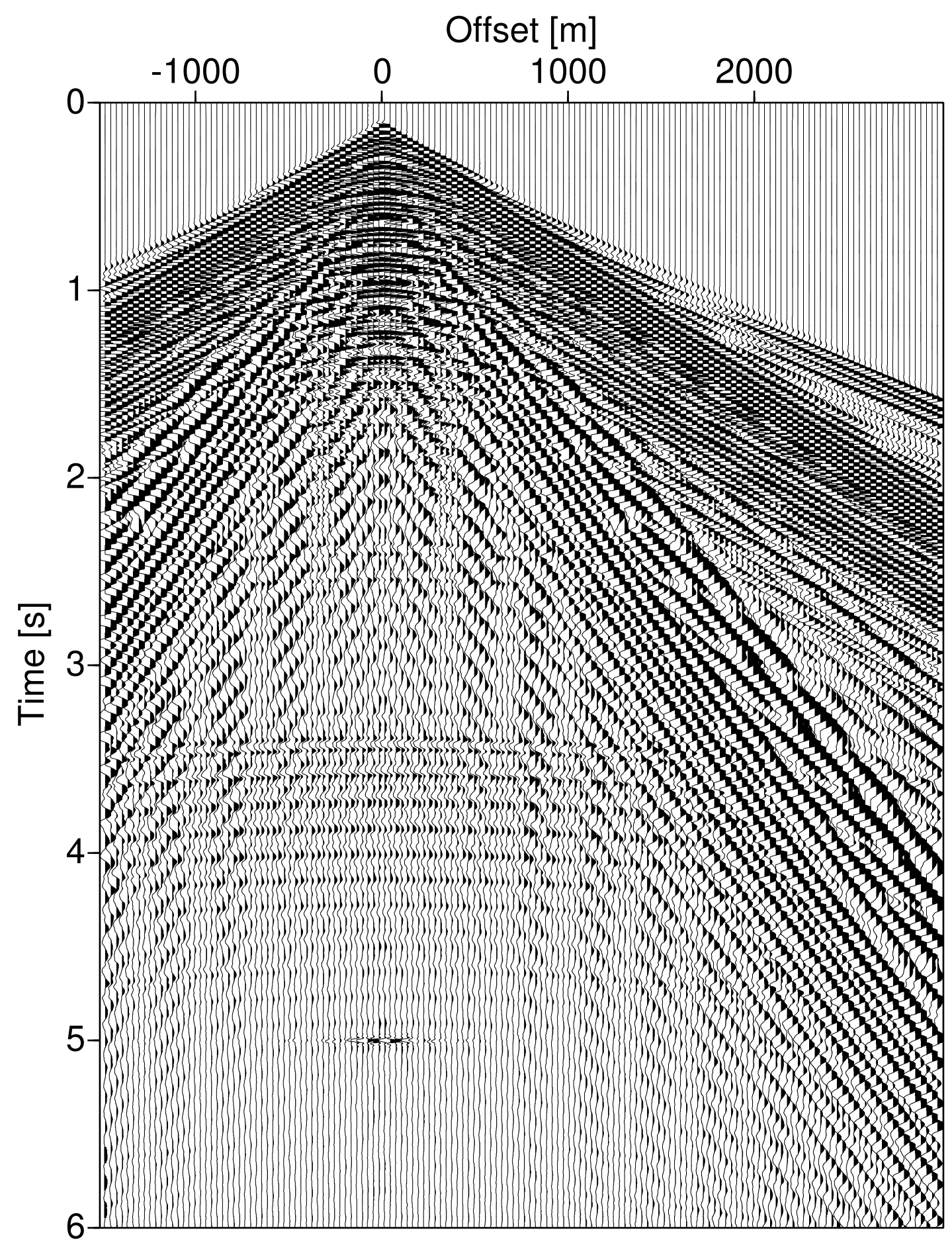

Fig. 4.16. Synthetic seismogram for Lake Maracaibo obtained using a layer configuration with a mud layer with gassy sediment and the seismic geometry acquisition split spread. The model configuration was composed with 15 layers. 

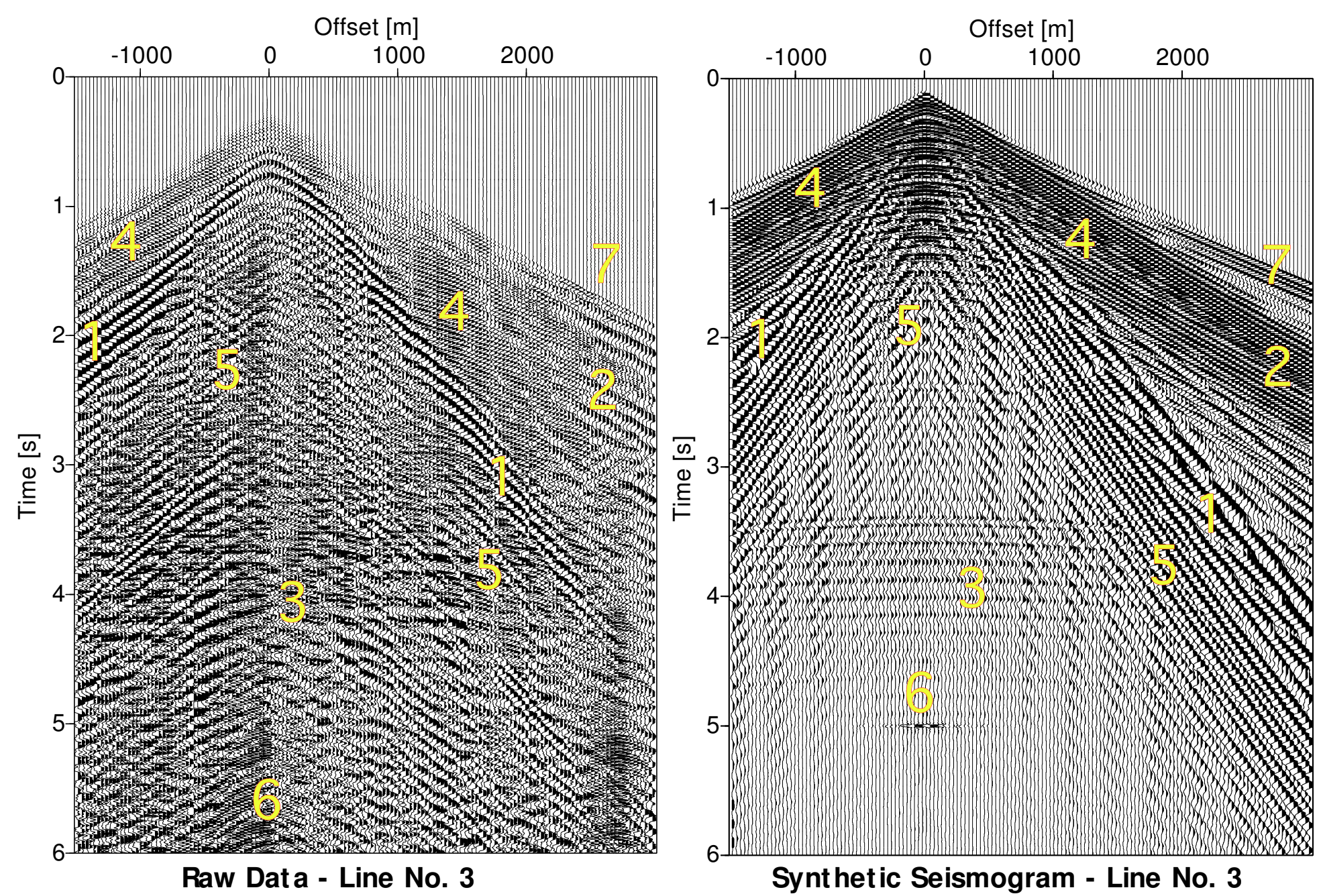

Fig. 4.17. Seismic data set from Lake Maracaibo in the mud layer area with gassy sediment and the synthetic seismogram built for this zone. Several similarities can be observed in both situations. 
From the above analysis of the raw data from the mud layer with gassy sediment and the synthetic seismogram built using the layers distribution shown in Figures 3.6 and 4.5, together with the seismic geometry acquisition shown in Figure 4.6, it is possible to verify that the most important factor producing the low signal/noise ratio and the ringing with low frequency content observed in the seismic data is the attenuation due to the gassy sediment present in the mud layer.

In order to prove that there is a rigid shale layer embedded in the mud layer zone with gassy sediment, a synthetic seismogram was built using the layer configuration described in Figure 4.5 and Figure 4.18. Figure 4.18 shows the layer configuration in the first $100 \mathrm{~m}$ in Lake Maracaibo, but without the rigid shale. In this case, the thickness of the mud layer was increased and the gassy sediments layer thickness was preserved.

Figure 4.19 shows the result of the modeling if the rigid shale is not considered when building the synthetic seismograms. The Stoneley wave has less velocity than in the model with the rigid shale and it is not possible to observe the destructive interference that is appreciable in the real data and the synthetic seismogram of this layer. Additionally, the dispersion behavior in the Stoneley wave is different in both cases.

This study showed that the attenuation quality factor $Q_{\alpha}$ that produces the best match between the real data and the synthetic seismogram is between 5 and 20. This attenuation is over the compressional wave and produced a very strong dispersion in the Stoneley wave. It is possible to observe constructive and destructive interference in this surface wave. Additionally, it was verified that the viscous losses, the solid friction and the mud layer effect, without gassy sediment, are other attenuation mechanisms present in this area. Therefore, it was observed that attenuation quality factors present in the zone without gassy sediment are very similar to the attenuation quality factors for the gassy sediment zone below the rigid shale layer. Table 4.3 shows the different parameters used in building the synthetic seismograms in the mud layer zone with gassy sediments.

The results show that the best match between the raw data and the synthetic seismogram was when the attenuation quality factor $Q$ was independent of frequency with $n$ equal to 0 in the frequency range between $1 \mathrm{~Hz}$ and $70 \mathrm{~Hz}$. When the value of $n$ was different than 0 , the synthetic seismogram showed unrealistic results. Figure 4.20 shows two synthetic seismograms with the same layer configuration, velocities, thick- 


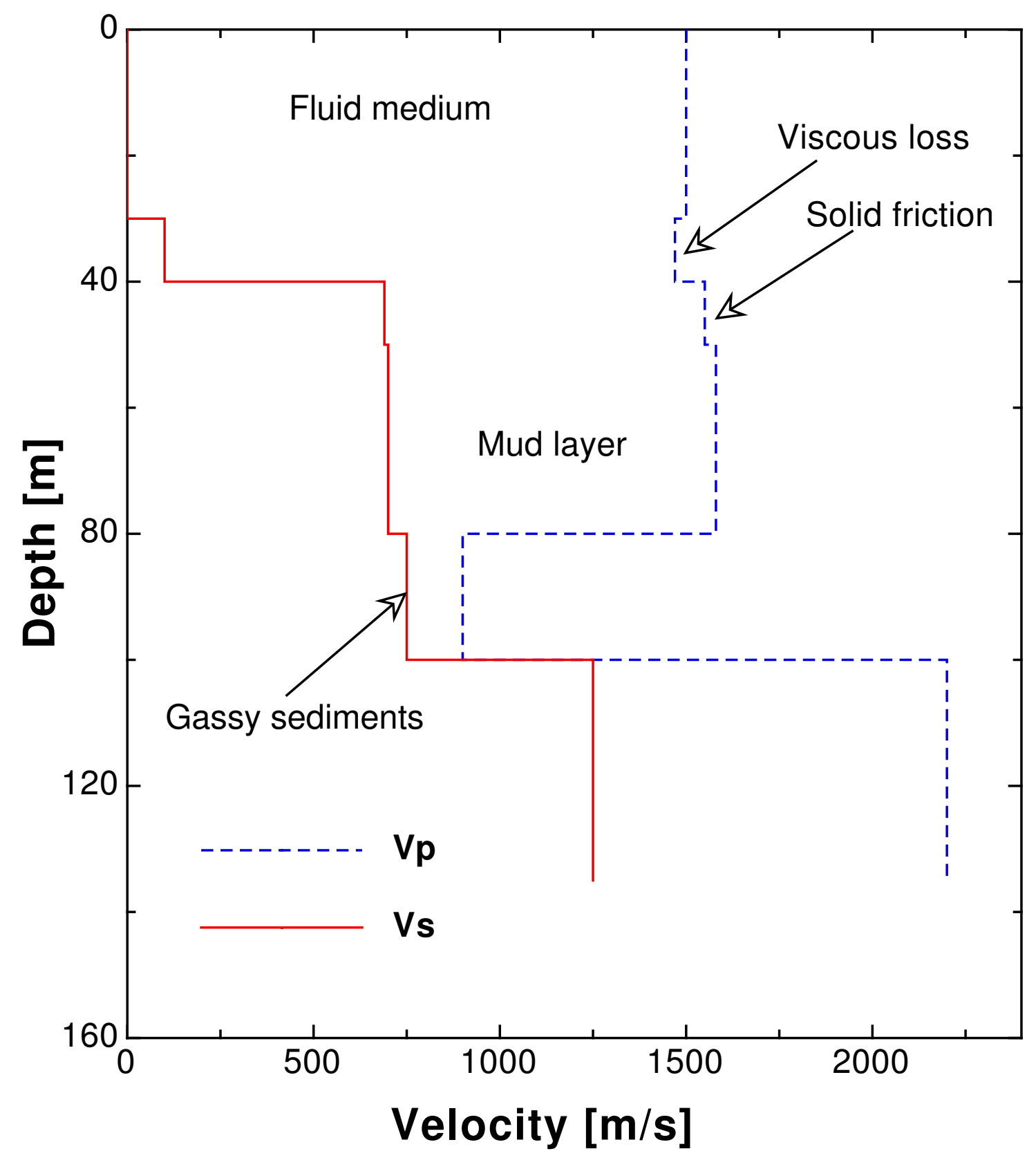

Fig. 4.18. Velocity profile used to build the synthetic seismogram in Lake Maracaibo for the mud layer zone, with gassy sediment, but without the rigid shale. Three different kinds of attenuation mechanisms are considered: viscous losses, solid friction losses and gassy sediments effect. 

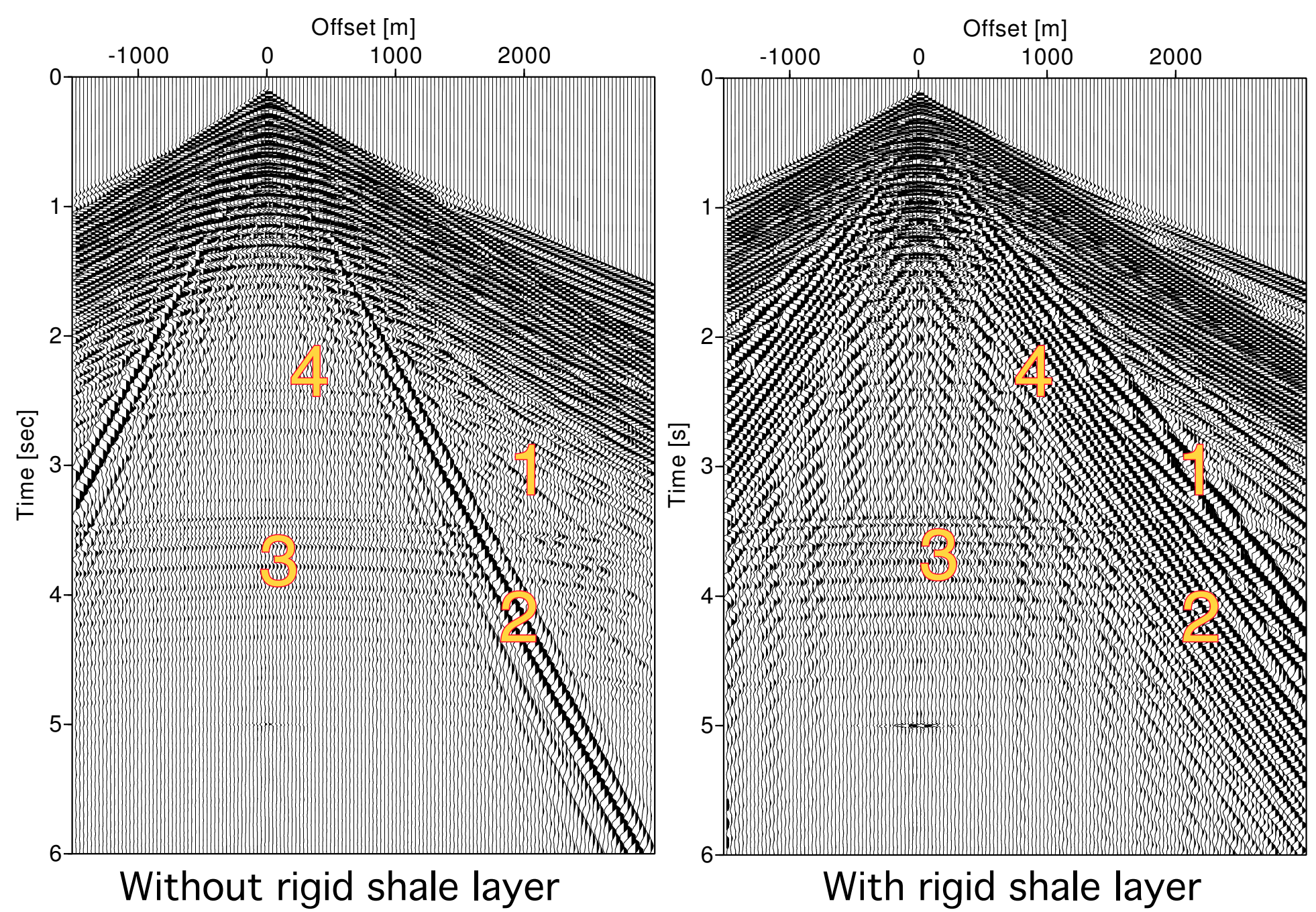

Fig. 4.19. Synthetic seismogram built for the mud layer with gassy sediment without and with the rigid shale. In this case, it is not possible to appreciate the destructive interference in the Stoneley wave in the synthetic seismogram without the rigid shale layer. 
Table 4.3. The different parameters used in building of the synthetic seismograms in the mud layer zone with gassy sediments in Lake Maracaibo.

\begin{tabular}{|c||c||c||c||c||c||c|}
\hline Layer & $Q_{\alpha}$ & $Q_{\beta}$ & $V_{p}[\mathrm{~m} / \mathrm{s}]$ & $V_{s}[\mathrm{~m} / \mathrm{s}]$ & $\rho[\mathrm{g} / \mathrm{cc}]$ & Thickness $[\mathrm{m}]$ \\
\hline \hline Fluid medium & 200 & - & 1500 & 0 & 1.10 & 30 \\
\hline Viscous medium & 110 & 20 & 1470 & 100 & 1.25 & 10 \\
\hline Solid friction medium & 90 & 80 & 1550 & 690 & 1.30 & 10 \\
\hline Mud layer & 90 & 80 & 1580 & 700 & 1.32 & 5 \\
\hline Rigid shale & 150 & 150 & 1950 & 1050 & 1.60 & 30 \\
\hline Gassy sediments & 5 & 200 & 850 & 700 & 1.20 & 15 \\
\hline Milagro & 160 & 120 & 2200 & 1250 & 1.70 & 400 \\
\hline Layer A & 160 & 120 & 2425 & 1420 & 2.20 & 2200 \\
\hline Layer B & 160 & 120 & 2550 & 1500 & 2.30 & 1150 \\
\hline Layer C & 160 & 120 & 2750 & 1650 & 2.50 & 200 \\
\hline Layer D & 160 & 120 & 3020 & 1775 & 2.60 & 200 \\
\hline Layer E & 160 & 120 & 3600 & 2100 & 2.65 & Half space \\
\hline
\end{tabular}

nesses, etc., but different $n$ values, for the gassy sediments zone. It is possible to appreciate that when the $n$ value is different than 0 , the result are unrealistic. This situation could be observed for $n$ values of 1 and 2 .

In summary, the results show that the configuration of the layer between the zone with gassy sediment and the zone without gassy sediments is different. The most important difference is the rigid shale layer embedded in the mud layer with gassy sediments. This could not be observed in the zone without gassy sediments. Also, different thicknesses in the first $100 \mathrm{~m}$ were observed in both cases. 



Fig. 4.20. Synthetic seismogram with the same layer configuration, velocities, thicknesses, densities, etc., but with different $n$ value, in the gassy sediments zone. In the figure on the right, the value for $n$ was 1 ; an the left, the value for $n$ was 0 . 


\section{CHAPTER V}

\section{ANALYSIS AND RESULTS}

In Lake Maracaibo, the acoustic and elastic properties of unconsolidated sediments, consolidated sediments and gassy sediments are signifiantly different. In unconsolidated sediments and consolidated sediments media, without gassy sediments, several physical factors cause variations in compressional and shear waves velocities.

The porosity stands out as the most important single factor, because the difference in compressibility between water and mineral grains is so great. Another important element is the rigidity in the sediment. Others, with less importance are the pressure, temperature, compressibility and differences in densities. In the first $50 \mathrm{~m}$ in Lake Maracaibo, it was assumed that the velocity of the compressional wave, between $30 \mathrm{~m}$ and $40 \mathrm{~m}$ deep, was less than the velocity of the compressional wave in water, and additionally, it was assumed that the amount of particles was enough to support low shear wave velocity (Hamilton, 1956). After $40 \mathrm{~m}$, the compressional wave velocity was set to be greater than the compressional wave in fluid. In this case, the Poisson's ratio was set to be between 0.38 and 0.45 in order to estimate the converted shear wave velocity generated in this zone.

In Lake Maracaibo it was evident that, in the zone without gassy sediments, the velocity and attenuation of seismic waves in unconsolidated and consolidated sediments are strongly dependent upon pore fluid content and the amount of the suspended particles. In general, the degree of wave interaction with fluids is determined by the amount of particles. In this way, it is well known that flatter pores are more sensitive to the details of the fluid and its ability to support or transmit compressional and shear load.

The primary source of fluid attenuation in porous media is relative motion between the solid and liquid. Such motion results in shearing stresses in the fluid, and consequently, viscous dissipation of mechanical energy. Therefore, the attenuation of waves in unconsolidated sediments, consolidated sediments media, without gassy sediments, depends on the mechanical properties of the fluid, the discrete particles and the skeletal frame formed by the assemblage of particles. Motion of the fluid relative to the frame and the relative motion of contiguous particles are two potential 
sources of energy dissipation in these kinds of media. Hence, the attenuation of waves in unconsolidated sediments, consolidated sediments media, without gassy sediments, may be regarded as the sum of loss caused by the fluid motion and the loss caused by the solid framework. These mechanisms were included to account for the attenuation of compressional and shear waves through the viscous losses between the particles and the fluid, and the solid friction losses between the particles. Additionally, the effect produced by the mud layer is a third mechanism to account for the attenuation in Lake Maracaibo in the zone without gassy sediments, and was considered.

The results show that the attenuation produced by the viscous loss in the converted shear wave velocity is the most important factor to be considered in the mud layer without gassy sediments. The attenuation quality factor $Q_{\beta}$ that generated the best match between the raw data and the synthetic seismogram was between 10 and 20. Therefore, the solid friction loss and the mud layer effect also generated important attenuation over the converted shear wave. The attenuation quality factor $Q_{\beta}$ for both media was between 50 and 60, allowing the best match between the seismic data and the synthetic seismogram, see Figure 4.8 and Table 4.2.

Different quality factor values were used to account for the attenuation originated by the viscous loss, solid friction and mud layer effect over the compressional wave. The results show that these values can be between 90 and 150, and the differences among them are not clear. Almost the same result was found when the quality factor $Q_{\alpha}$ was set between these two values. However, the best match between the seismic data and the synthetic seismogram was found when the value for $Q_{\beta}$ was set equal to 90 . Additionally, the attenuation quality factor $Q$ was independent of freqency, provided that the attenuation quality factors $Q_{\alpha}$ and $Q_{\beta}$ are independent of frequency. Furthermore, the results show that the attenuation quality factor $Q_{\alpha}$ was always greater than the attenuation quality factor $Q_{\beta}$ for the same layer. The best match between the real data and the synthetic seismogram is found when the attenuation quality factor $Q$ was independent of frequency with $n$ equal to 0 in the frequency range between $1 \mathrm{~Hz}$ and $70 \mathrm{~Hz}$, see Figure 4.9 .

Finally, the results confirm that waves traveling between the surface lake and the lake bottom generate a very significant amount of ringing in Lake Maracaibo in the zone without gassy sediments. The shallow water ringing tends to be strongly coherent in the vertical component and is easily observed in the raw data from the zone without gassy sediments, see Figure 4.1. Additionally, it was observed that in 
the zone without gassy sediments, the frequency content of the ringing was larger than the frequency content in the zone with gassy sediments. The difference was at least equal to $10 \mathrm{~Hz}$. In the seismic data from Lake Maracaibo, this ringing produces seismograms showing waves which could easily be mistaken for a long series of reflections from deep horizons. Table 5.1 shows the final results found in the modeling using layers configuration without gassy sediment in the mud layer zone. The attenuation quality factors $Q_{\alpha}$ and $Q_{\beta}$ that generated the best match between the real data and the synthetic seismogram, in the zone without gassy sediments, for the remaining layers were between 150 to 160 and 110 to 120 , respectively.

Table 5.1. Final results found in the modeling of the mud layer without gassy sediment in Lake Maracaibo. The results show several differences from the zone with gassy sediment.

\begin{tabular}{|c||c||c||c||c||c|}
\hline $\begin{array}{c}\text { Mud Layer Zone } \\
\text { Without Gassy Sediment }\end{array}$ & $Q_{\alpha}$ & $Q_{\beta}$ & $\begin{array}{c}V_{p} \\
{[\mathrm{~m} / \mathrm{s}]}\end{array}$ & $\begin{array}{c}V_{s} \\
{[\mathrm{~m} / \mathrm{s}]}\end{array}$ & $\begin{array}{c}\text { Thickness } \\
{[\mathrm{m}]}\end{array}$ \\
\hline \hline Fluid & 200 & 200 & 1500 & 0 & 30 \\
\hline Viscous loss & 110 & 20 & 1470 & 100 & 10 \\
\hline Solid Friction & 90 & 80 & 1550 & 690 & 10 \\
\hline Mud Layer & 90 & 80 & 1580 & 700 & 50 \\
\hline
\end{tabular}

When the gassy sediments are present in the mud layer in Lake Maracaibo, they dominate the acoustic characteritics of the sediments in this zone. Therefore, several acoustic modifications of the lake bottom are observed: compressional wave velocity is reduced below the value for water saturated sediment, attenuation of waves propagating through the gassy sediment is increased by several orders of magnitude in comparison with attenuation observed in the zone without gassy sediment, the Poisson's ratio values are between 0.10 and 0.15 , and acoustic reflection from the gassy sediment is increased.

In gassy sediment, the sediment's shear rigidity and frame bulk modulus will modify the compressional and shear wave velocities. Because gas is less dense than sediment or water, the bulk density of a mixture of gas and sediment will be less than the bulk density of the sediment only. In this way, the gas content will cause sediment expansion, a consequent reduction of grain contacts, and reduced strength, producing a decrease in the bulk density that results in the low propagation speed of 
the compressional wave through this medium. In other words, in gassy sediment, the decrease in velocity due to decreasing bulk density with decreasing brine saturation is at a much greater rate due to low density gas replacing much higher density brine. The effect of decreasing bulk density is not compensated for by decreasing fluid compressibility due to the very high compressibility of gas compared to the compressibility of brine (Domenico, 1976).

The attenuation in gassy sediment is caused by frame friction absorption, as for saturated sediments and scattering by the gas. Absorption is due to loss mechanisms associated with gas motion. In this way, the attenuation depends considerably on water saturation for two phase water/gas mixtures. As in the case of velocities, the essential parameter controlling attenuation is the effective pressure. A decrease in attenuation is normally observed with increasing pressure for compressional and shear waves. Additionally, the attenuation in gassy sediment also depends on the distribution of gas bubbles within the porous medium (Bourbie et al., 1987).

Anderson and Bryant (1990) mention that the combination of low compressional wave velocity and an increase in the attenuation behaviour strongly suggests a low shear modulus. When low frequency energy is propagated at a low compressional wave speed and the attenuation observed is strong in comparison to a zone without gassy sediments, this is an indication that the attenuation is probably dependent of frequency.

The results based on the model with gassy sediments in the mud layer showed several differences when compared to the model without gassy sediments. First is the low signal/noise ratio observed in the seismic data, and that is due to the attenuation of the compressional wave generated by the gassy sediment in the mud layer. This attenuation produced a large amount of ringing with low frequency content, in comparison with the ringing observed in the seismic data from the zone without gassy sediments. The attenuation quality factors $Q_{\alpha}$ and $Q_{\beta}$ that generated the best match between the real data and the synthetic seismogram, in the zone without gassy sediments for the remaining layers, were 110 and 150 respectively, see Figure 4.17 and Table 4.3.

The best attenuation quality factor $Q_{\alpha}$ value that produces the best match between the seismic data and the synthetic seismogram is between 5 and 10 . The relationship between the compressional and shear wave velocity through the $V_{p} / V_{s}$ ratio in the gassy sediment layer was between 1.15 and 1.20 . This ratio generates 
a destructive interference in the Stoneley wave with apparent velocity between 900 $\mathrm{m} / \mathrm{s}$ and $1150 \mathrm{~m} / \mathrm{s}$. The results show that the layer configuration has to be different between the zone without gassy sediment and the zone with gassy sediment. The most important difference is due to the rigid shale layer that can be observed embedded in the mud layer zone. This rigid shale layer generates, together with the gassy sediments layer, the Stoneley wave, see Figure 4.19 and Figure 4.17.

Finally, it was observed that the model with viscous losses, solid friction and mud layer media has to be considered, along with the attenuation in the zone with gassy sediments. The attenuation quality factors $Q_{\alpha}$ and $Q_{\beta}$ apparently have the same value as in the zone without gassy sediment. Table 5.2 shows the final results found in the modeling using layers configuration with gassy sediment in the mud layer zone. Similar to the mud layer without gassy sediments case, the attenuation quality factors $Q_{\alpha}$ and $Q_{\beta}$ that generated the best match between the real data and the synthetic seismogram, in the zone with gassy sediments, for the remaining layers, were between 150 to 160 and 110 to 120, respectively. Additionally, the results show that the best match between the real data and the synthetic seismogram is found when the attenuation quality factor $Q$ was independent of frequency with $n$ equal to 0 in the frequency range between $1 \mathrm{~Hz}$ and $70 \mathrm{~Hz}$, see Figure 4.20.

Table 5.2. Final results found in the modeling of the mud layer with gassy sediment in Lake Maracaibo. The results show that there are several differences compared to the zone without gassy sediment.

\begin{tabular}{|c||c||c||c||c||c|}
\hline $\begin{array}{c}\text { Mud Layer Zone } \\
\text { With Gassy Sediment }\end{array}$ & $Q_{\alpha}$ & $Q_{\beta}$ & $\begin{array}{c}V_{p} \\
{[\mathrm{~m} / \mathrm{s}]}\end{array}$ & $\begin{array}{c}V_{s} \\
{[\mathrm{~m} / \mathrm{s}]}\end{array}$ & $\begin{array}{c}\text { Thickness } \\
{[\mathrm{m}]}\end{array}$ \\
\hline \hline Fluid & 200 & 200 & 1500 & 0 & 30 \\
\hline Viscous loss & 110 & 20 & 1470 & 100 & 10 \\
\hline Solid Friction & 90 & 80 & 1550 & 690 & 10 \\
\hline Mud Sediments & 90 & 80 & 1580 & 700 & 5 \\
\hline High Velocity Layer & 150 & 150 & 1950 & 1050 & 30 \\
\hline Gassy Sediment Layer & 5 & 200 & 850 & 700 & 15 \\
\hline
\end{tabular}




\section{CHAPTER VI}

\section{CONCLUSION}

The velocity profile used in the water unconsolidated sediments medium and the water consolidated sediments medium in the first $100 \mathrm{~m}$ in Lake Maracaibo followed the results of different studies considering these kinds of media (Hamilton, 1956; Shumway, 1960; Sarmiento and Kirby, 1962; Hampton, 1967; Stoll and Bryan, 1969; Hamilton, 1980; Bowles, 1997). These studies showed that the compressional wave velocity increases as porosity decreases, but this relationship is not linear. This finding was the most important considered with the velocity information from Lake Maracaibo provided by Sarmiento and Kirby (1962) in the definition of the velocity profile.

The attenuation of compressional and shear waves in rocks strongly depends on the physical state and saturation conditions. Therefore, the characteristics of compressional and shear waves propagating in an unconsolidated sediments medium or consolidated sediments medium, without gassy sediments, depend on the mechanical properties of the fluid, of the discrete particles and of the skeletal frame formed by the assemblage of particles. The motion of the fluid relative to the frame and the relative motion of contiguous particles are two potential sources of energy dissipation. In Lake Maracaibo, the characteristics of waves traveling through the lake layers were studied using the viscous losses between the particles and the fluid, the solid friction losses between particles, and the mud layer effect. Therefore, these three different mechanisms were proposed to account for the attenuation of the compressional and shear waves in the mud layer zone without gassy sediments.

The different results in the modeling using a layers configuration without gassy sediments in the mud layer zone showed that the attenuation quality factor $Q$ was independent of frequency with $n$ equal to 0 in the frequency range between $1 \mathrm{~Hz}$ and $70 \mathrm{~Hz}$, for both compressional and shear waves. This value generated the best match between the seismic data and the synthetic seismogram. These results implied that the attenuation quality factors $Q_{\alpha}$ and $Q_{\beta}$ were independent of frequency.

The results in the modeling using the layer configuration without gassy sediments in the mud layer showed that attenuation in this zone is mainly due to the viscous 
losses, solid friction and the mud layer effect. In this case, the attenuation quality factor for the shear wave was the most important factor to be considered. The models showed that the value for $Q_{\beta}$ between 10 and 20 produced the best match between the seismic data and the syntheic seismogram. In the same way, the best value for $Q_{\alpha}$ was between 90 and 150. The velocity range for the shear wave in the viscous losses was between $100 \mathrm{~m} / \mathrm{s}$ and $200 \mathrm{~m} / \mathrm{s}$, with compressional wave velocity between $1450 \mathrm{~m} / \mathrm{s}$ and $1480 \mathrm{~m} / \mathrm{s}$. In the solid friction and mud layer case, compressional and shear waves were very similar. These three media together generated the main features observed in the seismic data: Stoneley wave, ringing, direct wave, head wave, spacial aliasing, etc.

When the gassy sediments are present, even in small quantity, the attenuation generated by this condition is dominant over the other attenuation mechanisms, including the viscous loss, solid friction loss and mud layer effect. This is because bubble gas in resonance is a very strong attenuator of compressional waves. Additionally, the studies made using the layer configuration with gassy sediments in the mud layer showed that the best result was obtained when the attenuation quality factor $Q$ was independent of frequency.

The dependence of compressional and shear waves' velocities on saturation is very slight, for gas saturations between $10 \%$ and $90 \%$. Consequently, velocities are not good indicators of the quantity of gas present in the pores. By constrast, acoustic attenuation is highly sensitive to different amounts of gas, and several studies show that the attenuation reaches a peak for gas saturation from $20 \%$ to $40 \%$. This fact was evident in the study of the attenuation over the seismic data from Lake Maracaibo. In this case, the value of the attenuation quality factor $Q_{\alpha}$ that generated the best match between the raw data and the synthetic seismogram was between 5 to 10 with an attenuation quality factor $Q_{\beta}$ larger than 200. Several models showed that a small change in the attenuation quality factor $Q_{\alpha}$ generates more changes in the synthetic seismogram than larger changes in the velocity profile.

In this study, in almost all the cases, the attenuation quality factor $Q_{\alpha}$ was larger that the attenuation quality factor $Q_{\beta}$. The only exception was the gassy sediment case where the $Q_{\beta}$ was larger than $Q_{\alpha}$. Additionally, the attenuation quality factor $Q_{\alpha}$ was affected by the distribution of gas bubbles within the porous medium. This was evident when the thickness of the gassy sediment was modified. In this case, the effect of the attenuation quality factor $Q_{\alpha}$ increased, over the seismic data, when the 
thickness of the gassy sediment increased.

The results show that in the mud layer with gassy sediments, the best match between the raw data and the synthetic seismogram was found when an embedded layer of rigid shale was located within the mud layer. This layer was located between the mud sediments layer of rigid shale and the gassy sediments layer. The compressional velocity was between $1850 \mathrm{~m} / \mathrm{s}$ and $2050 \mathrm{~m} / \mathrm{s}$; shear wave velocity was between $950 \mathrm{~m} / \mathrm{s}$ and $1150 \mathrm{~m} / \mathrm{s}$, and the thickness greater than $30 \mathrm{~m}$. The compressional wave velocity for the mud sediment was between $1450 \mathrm{~m} / \mathrm{s}$ and $1650 \mathrm{~m} / \mathrm{s}$, and the shear wave velocity was between $600 \mathrm{~m} / \mathrm{s}$ and $800 \mathrm{~m} / \mathrm{s}$. The compressional wave velocity in the gassy sediments layer was between $800 \mathrm{~m} / \mathrm{s}$ and $900 \mathrm{~m} / \mathrm{s}$, and the shear wave velocity was between $680 \mathrm{~m} / \mathrm{s}$ and $780 \mathrm{~m} / \mathrm{s}$. The best result was found with a thickness for the gassy sediments layer between $12 \mathrm{~m}$ and $17 \mathrm{~m}$. These layers generate the Stoneley wave with an apparent velocity between $900 \mathrm{~m} / \mathrm{s}$ and $1150 \mathrm{~m} / \mathrm{s}$ and cause a destructive interference in this wave, which is evident in the field data. 


\section{REFERENCES}

Aki, K., and Richards, P. G., 1980, Quantitative seismology, theory and methods, Volume I: W. H. Freeman and Company.

Anderson, A. L., and Bryant, W. R., 1990, Gassy sediment occurrence and properties: Northern Gulf of Mexico: Geomarine Letters, 10, 209-220.

Anderson, A. L., and Hampton, L. D., 1980a, Acoustics of gas-bearing sediment I. Background: Journal of Acoustical Society of America, 67, 1865-1890.

1980b, Acoustics of gas-bearing sediments II. Measurements and models: Journal of Acoustical Society of America, 67, 1891-1904.

Bourbie, T., Coussy, O., and Zinszner, B., 1987, Acoustic of porous media: Editions Technip.

Bowles, F. A., 1997, Observations on attenuation and shear-wave velocity in finegrained, marine sediments: Journal of Acoustical Society of America, 101, 33853397.

Burg, K. E., Ewing, M., Press, F., and Stulken, E. J., 1951, Seismic wave guide phenomenon: Geophysics, 16, 594-611.

Domenico, S. N., 1976, Effect of brine-gas mixture on velocity in an unconsolidated sand reservoir: Geophysics, 41, 882-894.

Edrington, T. S., and Callowat, T. M., 1984, Sound speed and attenuation measurements in gassy sediments in the Gulf of Mexico: Geophysics, 49, 297-299.

Gassmann, F., 1951, Elastic waves through a packing of spheres: Geophysics, 16, 673-685.

Gibson, R. L., and Toksöz, M. N., 1989, Viscous attenuation of acoustic waves in suspensions: Journal of Acoustical Society of America, 85, 1925-1934.

Hamilton, E. L., Shumway, G., Menard, H. W., and Shipek, C. J., 1955, Acoustic and other physical properties of shallow-water sediments off San Diego: Journal of Acoustical Society of America, 28, 1-15. 
Hamilton, E. L., 1956, Low sound velocities in high porosity sediments: Journal of Acoustical Society of America, 28, 16-19.

1972, Compressional wave attenuation in marine sediments: Journal of Acoustical Society of America, 37, 620-646.

1980, Geoacoustic modeling of the sea floor: Journal of Acoustical Society of America, 68, 1313-1340.

Hampton, L. D., 1967, Acoustic properties of sediments: Journal of Acoustical Society of America, 42, 882-890.

Lay, T., and Wallace, T. C., 1995, Modern global seismology: Academic Press.

Levin, F. K., 1962, The seismic properties of Lake Maracaibo: Geophysics, 27, 35-47.

Mandal, B., and Mitchell, B. J., 1986, Complete seismogram synthetic for transversely isotropic media: Journal of Geophysics, 59, 149-156.

McCann, C., and McCann, D., 1969, The attenuation of compressional waves in marine sediments: Geophysics, 34, 882-892.

Michelena, R. J., Mora, P. J., Alvarellos, J., and Malave, G., 1998, Sismica experimental en zonas de baja calidad de datos en el Lago de Maracaibo: enfoque integral: IX Congreso Venezolano de Geofisica, 1, 1-17.

Sarmiento, R., and Kirby, R. A., 1962, Recent sediments of Lake Maracaibo: Journal of Sedimentary Petrology, 32, 698-724.

Sheriff, R. E., and Geldart, L. P., 1995, Exploration seismology: Cambridge University Press.

Shumway, G., 1960, Sound speed and absorption studies of marine sediments by a resonance method, Part II: Geophysics, 25, 659-682.

Stockwell, J. W., and Cohen, J. K., 2000, The new SU user's manual: The Gas Research Institute.

Stoll, R. D., and Bryan, G. M., 1969, Wave attenuation in saturated sediments: Journal of Acoustical Society of America, 1, 1440-1447. 
Toksöz, M. N., and Johnston, D. H., 1981, Seismic wave attenuation: Society of Exploration Geophysicists.

Urick, R. J., 1948, The absorption of sound in suspensions of irregular particules: Journal of Acoustical Society of America, 20, 283-289.

Wood, A. B., and Weston, D. E., 1964, The propagation of sound in mud: Acoustic, 14, 156-162.

Wyllie, M. R. J., Gardner, G. H., and Gregory, A. R., 1962, Studies of elastic wave attenuation in porous media: Geophysics, 27, 569-589. 


\section{VITA}

\section{Javier Antonio Pérez Arredondo}

Department of Geology and Geophysics

Texas A\&M University

College Station, Texas 77843

979-458-1169

\section{Education}

M.S., December 2002, Texas A\&M University, Department of Geology and Geophysics.

B.S., July 1997, Universidad del Zulia, Department of Geodesy, Maracaibo Estado Zulia, Venezuela. Major: Geodesy.

\section{Permanent address}

Urbanizacion La Rotaria

Av. 85, No. 82-23

Frente a la Panaderia La Fuente

Maracaibo, Estado Zulia

Venezuela

(58) 212-383-9415

email: perezjzy@pdvsa.com 